University of Louisville

ThinkIR: The University of Louisville's Institutional Repository

Electronic Theses and Dissertations

8-2010

\title{
Electron emission from nanostructured materials.
}

Abdelilah Safir

University of Louisville

Follow this and additional works at: https://ir.library.louisville.edu/etd

\section{Recommended Citation}

Safir, Abdelilah, "Electron emission from nanostructured materials." (2010). Electronic Theses and Dissertations. Paper 1246.

https://doi.org/10.18297/etd/1246

This Doctoral Dissertation is brought to you for free and open access by ThinkIR: The University of Louisville's Institutional Repository. It has been accepted for inclusion in Electronic Theses and Dissertations by an authorized administrator of ThinkIR: The University of Louisville's Institutional Repository. This title appears here courtesy of the author, who has retained all other copyrights. For more information, please contact thinkir@louisville.edu. 


\title{
ELECTRON EMISSION FROM NANOSTRUCTURED MATERIALS
}

\author{
By
}

\begin{abstract}
Abdelilah Safir
B.S. HassanII University, 1996

M.S. HassanII University, 1999

M.S University of Louisville, 2002
\end{abstract}

\begin{abstract}
A Dissertation
Submitted to the Faculty of the

Graduate School of the University of Louisville in Partial Fulfillment of the Requirements

for the Degree of
\end{abstract}

\author{
Doctor of Philosophy \\ Electrical and Computer Engineering Department \\ University of Louisville \\ Louisville, Kentucky
}

August, 2010 
Copyright 2010 by Abdelilah Safir

All rights reserved 



\title{
ELECTRON EMISSION FROM NANOSTRUCTURED MATERIALS
}

$$
\text { By }
$$

\author{
Abdelilah Safir \\ B.S. HassanII University, 1996 \\ M.S. HassanII University, 1999 \\ M.S University of Louisville, 2002
}

\begin{abstract}
A Dissertation Approved on
July 26,2010

by the following Dissertation Committee:

Robert W. Cohn (Dissertation Director)

Gamini $\mathrm{H}$. Sumanasekera (Co-advisor)

Bruce W. Alphenaar

Cindy Harnett
\end{abstract}

Frank P. Zamborini 


\section{DEDICATION}

This dissertation is dedicated to my parents

\section{Mrs. Fatna Elghlia Sahli}

and

\section{Mr. M'Barek Safir}

Who have given me invaluable educational opportunities. 


\section{ACKNOWLEDGMENTS}

Firstly, I would like to express my gratitude, thanks and praise to my creator Allah (GOD) who provides me with the intellect, the means of learning and everything that I am in need of.

I would like to express my appreciation and gratitude to my advisor Dr. Robert W. Cohn, for his continuous help, insight, enthusiasm, excellent guidance and advice throughout my work at ERINC. I really cannot find the proper words to thank him enough for his excellent and inspiring guidance and for his helpful moral support during all these years. I would like to thank you Dr. Cohn for giving me the chance to work at ERINC where I've learned how to tread through the nanotechnology and metrology fields...

I owe my deepest gratitude to my co-advisor Dr. Gamini U. Sumanasekera. My research for this dissertation was made more efficient but also much more extensive through the use of Dr. Sumanasekera's lab and valuable discussions. Thus, I gladly express my gratitude to Dr. Gamini U. Sumanasekera for his monitoring and advice through the course of this work.

I would like to thank my other dissertation committee members Dr. Bruce W. Alphenaar, Dr. Cindy Harnett and Dr. Frank P. Zamborini for revising my dissertation and for their valuable support and advice. 
This dissertation would not have been possible unless I had received good samples from Dr. Mahendra K Sunkara and Rupa Dumpala, Dr. Gamini U. Sumanasekera, Dr. Tereza Paronyan, and Chaminda Jayasinghe. Moreover, my research was much easier with the continuous help and valuable support received from Joseph Williams. Thank you all.

I am indebted to David Mudd for his valuable participation and for being the best co-worker I could have ever had the pleasure of working with during this study; thank you David. Also, I would like to show my gratitude to Dr. Mehdi Yazdanpanah for his help and support from the day I joined ERINC until the day of writing these lines. I would like to thank Dr. Vladimir Dobrokhotov for his significant contribution to this work through his supervision and advice.

I would like to take this opportunity to thank my friends, colleagues and all those who have contributed in any way to the completion of this work, especially, Abdelmoula Haboub, Nasr Eddine Yahyauoi, Robert Stallard for reading and editing this dissertation, Santosh Pabba, Dr. Brigitte H. Totten Dr. Kapila Hewaparakrama, Harut Vardanyan, Dr. Sharvil Desai, Dr. Adam Willitsford, Douglas Jackson, Mark Crain, Scott Cambron, Thomas Roussel. I am also thankful to all University of Louisville faculty and staff and for the great assistance from numerous librarians.

Thank you to my brothers and sisters, Fatima, Rachida, Mohamed, Fatiha, Mahjouba, Siham, Sanaa and Hanan for the continuous love and support they have given me throughout my life. Special thanks to my brother and friend Abdelmajid Safir for his continuous love, concern and support. I also wanted to thank all of my nieces and 
nephews for their love.

Special thanks to my children Rahaf and AbdouAllah and to my wife, Ibtissam Labbardi, who has completed my life and has been helping me in every phase of my graduate career.

I am grateful to my parents, Mrs. Fatna Elghalia Sahli and Mr. M'barek Safir, who have provided me with continuous love, support, and encouragement throughout my life. They have given me everything that I could ever need to succeed and I am forever in their debt. 


\section{ABSTRACT \\ ELECTRON EMISSION FROM NANOSTRUCTURED MATERIALS}

Abdelilah Safir

July 26,2010

In this dissertation, standardized methods for measuring electron emission (EE) from nanostructured materials are established. Design of an emitter array platform, synthesis and nanomanipulation of different types of are successfully conducted. Preexisting as well as novel nanostructures are examined for possible use as electron point sources. Three main categories of emitters are under evaluation: oxide nanowires, metallic nanowires and carbon based nanomaterials (CBNs). Tungsten oxides nanowires have low work function, then metallic nanowires have high electrical conductivity and abundant number of free electrons at and below their Fermi level and lastly, CBNs have superior electrical, mechanical, chemical and thermal properties. This evaluation is designed to compare and choose among the nanoemitters that are suitable for EE.

Simulation through theoretical modeling is provided to optimize the parameters directly or indirectly affecting EE properties. The models are to enhance the emitter's performance through increase the packing density, reduce the field screening effect, lower the turn-on and the threshold electric fields and increase the emission current densities. The current estimations and the modeling of the validity regions where EE types theoretically exist, help to select and fabricate optimum emitters. 
An assembly consisting of sample substrate, electrical feedthroughs, electrodes, nano/micro-manipulator and insulators are mounted within a vacuum chamber. An ion vacuum pump and a turbo pump are used to reach a vacuum pressure of $10^{-7}$ Torr. Two systems are used for EE characterization of nanostructures: bulk and In-situ configurations. The bulk investigation is realized by designing a vacuum chamber and different sample holders that can resist harsh environment as well as high temperature for both FE and TE experiments. In-situ experiments are conducted in the chamber of the scanning electron microscope (SEM), it consists of designing special sample holders plus modifications of the SEM chamber for the ease of EE characterization.

Samples with different materials, densities, radii of curvatures, and lengths ranging respectively from $10^{7}-10^{9} \mathrm{emitter} / \mathrm{cm}^{2}, 5-300 \mathrm{~nm}$, and $3 * 10^{3}-10^{7} \mathrm{~nm}$, are produced. The CBNs used are characterized by different structures and shapes that are defined by the monolayer carbon sheet takes. Cylindrical sheets are equivalent to nanotubes while graphene are flat sheets. Emitter's structures are varied by altering the critical growth parameters such as temperature, pressure and constituent materials. Enhancement of the FE properties, the design of an optimum emitter density and reduction of the field screening effect is possible by selecting appropriate materials, synthesizing nanostructures with small radius $(10 \mathrm{~nm})$, high aspect ratio (greater than 1000), the ideal density where the inter-emitter distance is comparable to the emitter height, the cathodes' uniformity, the treatment of the emitting surface, and integrating triode arrangement.

Initially, the thermionic Emission (TE) investigations of these nanostructures produce emission at an onset temperature of $500{ }^{\circ} \mathrm{C}$, current densities of $160 \mathrm{~mA} / \mathrm{cm}^{2}$ at 
temperatures of $700-1200{ }^{\circ} \mathrm{C}$ and predict the work function of the emitting materials. In addition, nanostructures can enhance the local electric field and increase the packing density to produce better EE properties.

Then, FE investigations from different nanostructures showed that the small tip's diameter, high aspect ratio and tapered structures enhance emission through low turn-on fields $(<0.8 \mathrm{~V} / \mu \mathrm{m})$, low threshold fields $(<3 \mathrm{~V} / \mu \mathrm{m})$ and high current densities $(520$ $\mathrm{mA} / \mathrm{cm}^{2}$ ). CCNTs having inter-emitters distance comparable to their average height contribute to the reduction of the field screening effect through large field enhancement factor $\beta$ (> 7000) and enhancement of the EE properties.

EE experimental data along with its analysis demonstrate that CBNs have lower turn-on electric field, lower threshold fields, higher current density and higher field enhancement factor than those of microscopic metallic cathodes and oxide nanowires. Therefore, nanomaterial based emitters with their superior intrinsic properties based on the achieved EE results can be turned into potential EE point sources. 


\section{TABLE OF CONTENTS}

\section{PAGE}

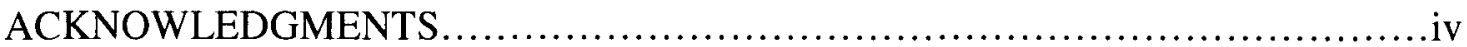

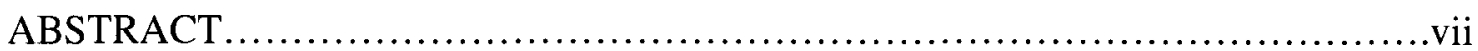

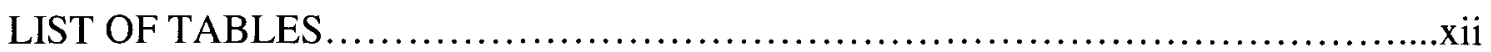

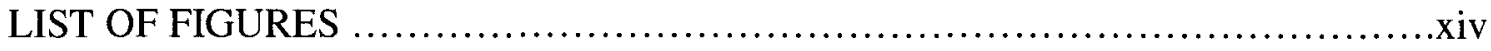
CHAPTER

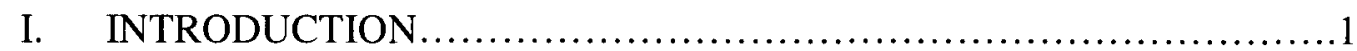

II. THEORETICAL BACKGROUND AND ESTIMATIONS ..................24

III. EXPERIMENTS ..................................................59

IV. RESULTS AND ANALYSIS: Thermionic emission.......................82

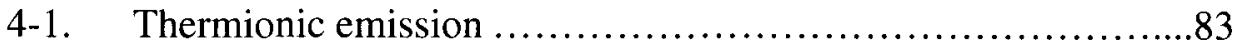

4-2. Thermionic emission from Conical carbon nanotubes...........85

4-3. Thermionic emission from semiconducting MWCNTs.........95

4-4. Thermionic emission from Metallic SWCNTS.................99

4-5. Comparison...........................................104

V. RESULTS AND ANALYSIS: Field emission ............................. 106

5-1. Characterization of nanowires ................................... 108

5-2. Characterization of Semiconducting MWCNTs .....................110

5-3. Characterization of CCNTs....................................... 115

5-3-1. Bulk characterization........................................115

5-3-2. In-situ characterization of CCNTs...............................125 
5-4. FE characterization of M_SWCNTs ..............................130

5-5. FE characterization of graphene .....................................132

5-6. Comparison of FE properties of CBNs ...............................133

VI. SUMMARY AND CONCLUSIONS .................................. 137

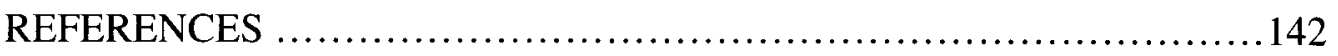

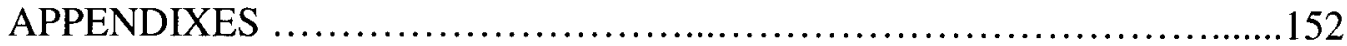

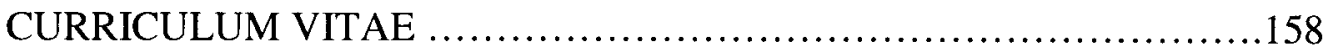




\section{LIST OF TABLES}

TABLE $\quad$ PAGE

1-1. A comparison of VME devices and solid-state devices properties ...................15

2-1. The work functions and TE properties of tungsten, thoriated tungsten and

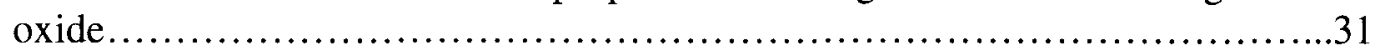

2-2. A selection of frequently used TE materials.............................51

2-3. Effect of work function and temperature on the TE current properties ........51

4-1. The structural characteristics, the FETE and TE properties of CCNTs array.......93

4-2. The structural characteristics, the FETE and TE properties of MWCNTs carpet

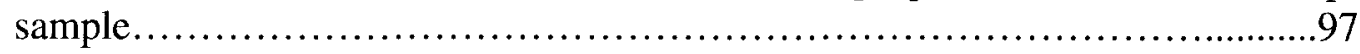

4-3. The structural characteristics, the FETE and TE properties of the M_SWCNTs film ...................................................................

4-4. A Comparison of the TE and FETE properties and the structural characteristics of

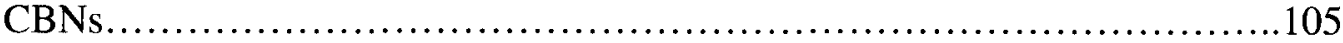

5-1. Experimental conditions, the resulting structural characteristics, and the field emission properties of three CCNTs array samples..........................117

5-2. Comparison of the resulting structural characteristics and the field emission properties of two CCNTs................................................ 124

5-3. Experimental conditions, the resulting structural characteristics, and the field emission properties of four individual CCNTs array samples...............127

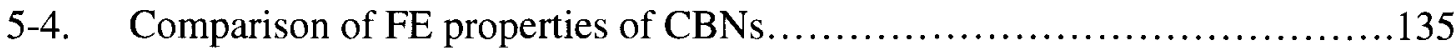

6-1. EE properties obtained for nanostructured materials during the present work ...140

6-2. The outcome of EE characterization from nanostructured materials ...................141 


\section{LIST OF FIGURES}

FIGURE

PAGE

1-1. (a) A schematic of EE from a tapered conductor caused by an external excitation. (b) Three different kinds of EEs that take place once the solid is subjected to an external excitation........................................ 3

1-2. The different processes of EE taking place within the metal-vacuum interface once external excitations are present. . .4

1-3. A schematic of the potential barrier for three different metals without the presence of an applied electric field.

1-4. A schematic of the potential barrier existing within the metal with and without the presence of an applied electric field. .9

1-5. The effect of field enhancement on the potential barrier.

1-6. A schematic of FEAs consisting of regular arrays in which cones are placed in small empty cylindrical spaces in an oxide film .13

2-1. A schematic of the potential barrier seen by an electron present within the conductor 25

2-2. The Fermi-Dirac distribution function $f(E)$ versus the energy $\left(E-E_{F}\right)$ for different temperatures .26

2-3. The emission current density versus temperature .29

2-4. A schematic of one dimensional energy band diagram in the absence of electric fields 30

2-5. A schematic of a one dimensional energy band diagram .....................33

2-6. The electric field lines near a flat surface and a sharp tip .......................

2-7. The circuitry of the field emission system set-up: triode configuration ........39

2-8. The emission current of a tungsten filament and treated tungsten as

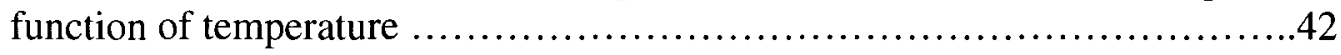

2-9. Validity regions of electron emission for a $5 \mathrm{eV}$ work function cathode ....... 53 
2-10. FE boundaries for five different materials TE ad ScE boundaries for five different materials

2-11. Thermionic emission and Schottky emission boundaries for five different materials.

2-12. Simulation of the equipotential lines and the electrostatic field distribution at the

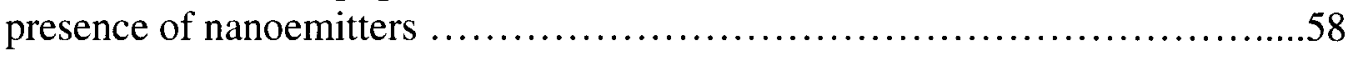

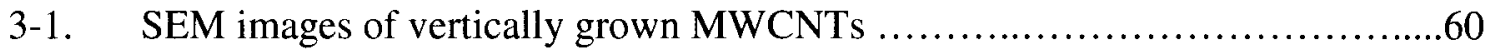

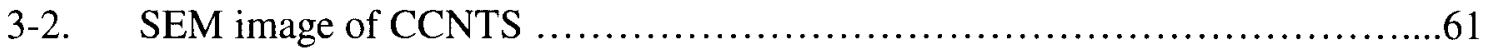

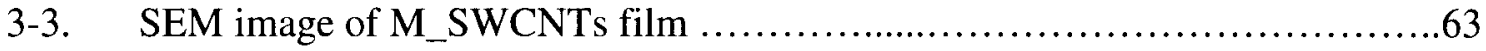

3-4. The Raman spectra of M_SWCNTs .......................................65

3-5. SEM image of graphene sheets deposited on a silicon .......................67

3-6. Time-lapse SEM images of needle formation .............................69

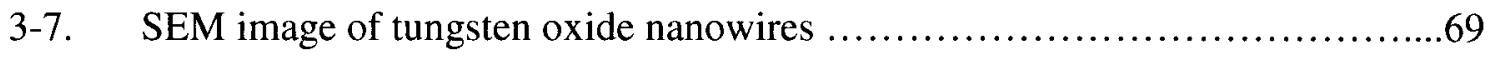

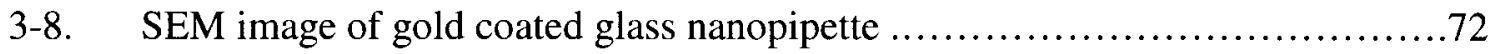

3-9. A Schematic of the electrochemical cell and SEM image of STM tip ...........73

3-10. (a),(b) Pictures of the EE set-up (c) SEM image of the anode-cathode (d) Schematic of the diode configuration ....................................75

3-11. Schematic of FE and TE bulk characterization system and sample

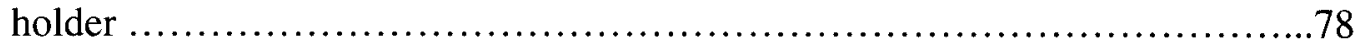

3-12. Schematic and SEM image of diode configuration for In-situ FE characterization and the Zyvex nanomanipulation system .................................80

4-1. SEM images of CCNTs sample (a) arrays of tips (b) zoom-in image of individual

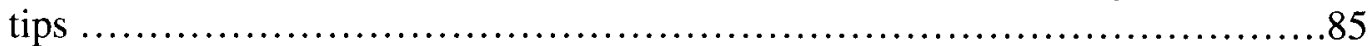

4-2. Emission current versus applied voltage of CCNTs at two different temperatures..........................................................86

4-3. FETE I-V curves of the CCNTs arrays at various temperatures in linear scale and

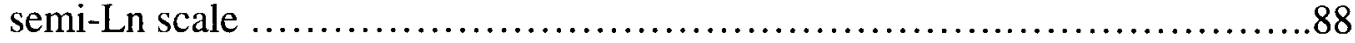

4-4. Thermionic emission characteristics of CCNTs and Richardson plots ..........89

4-5. CCNTs UPS spectra obtained ............................................. 
4-6. I-V characteristics of CCNTs sample at two different separations ..............94

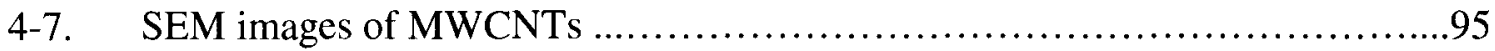

4-8. Thermionic emission characteristics and Richardson plots of MWCNTs .......97

4-9. SEM image of a M_SWCNTs film .......................................99

4-10. FETE I-V curves of the CCNTs arrays at various temperatures in linear scale and semi-Ln scale

4-11. The measured FETE I-V curves of the CCNTs arrays at various temperatures in in semi-Ln scale 101

4-12. Thermionic emission characteristics and Richardson plots of MWCNTs .......102

5-1. Field emission characteristics and the corresponding F-N plot of a tungsten oxide nanowires carpet....................................................... 109

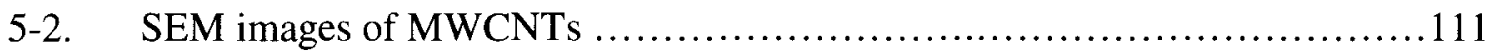

5-3. I-V curves of a super long carpet of MWCNTs........................... 111

5-4. I-V curves and the corresponding F-N plot of a super long MWCNT carpets at

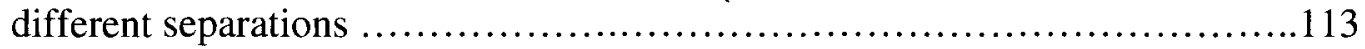

5-5. I-V curves of a short MWCNTs carpet at different distances and the emission current versus time.................................................114

5-6. SEM images of three samples of CCNTs ..................................... 118

5-7. SEM images of individual CCNTs illustrating the variation in the wall structure

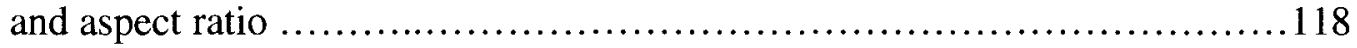

5-8. Field emission properties from the CCNTs showing the plots of the current vs. the macroscopic electric field for various separations and the corresponding F-N plots 120

5-9. Plots of the field enhancement factor $\beta$ and turn-on electric field as a function of distance for samples $1-3$ 121

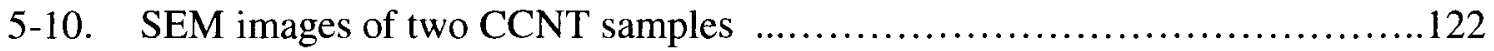

5-11. Current vs. the macroscopic electric field and the corresponding F-N plots ...123

5-12. SEM image of a single CCNT with STM tip as counter electrode in a diode configuration and the I-V characteristics at $50 \mu \mathrm{m}$ separation ................ 125

5-13. I-V curves and the corresponding F-N plot of a single CCNT ..................126 
5-14. I-E characteristics of an individual CCNT at $100 \mu \mathrm{m}$ separation.................129

5-15. Emission current from M_SWCNTs versus applied field in linear-scale and Logscale with the corresponding F-N plot for .................................130

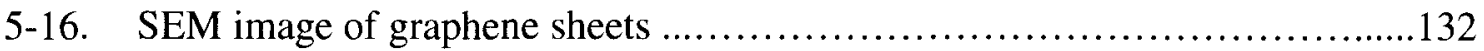

5-17. I-E curves and the corresponding F-N plot for graphene and emission versus time of HOPG 


\section{CHAPTER I \\ INTRODUCTION}

Nanomaterials emergent within Vacuum Microelectronics (VME) revived the field of vacuum devices and opened new applications from which a new industry may appear - especially where semiconductor based devices need to be more tolerant to harsh environments. Unlike the existing solid state devices, VME devices have faster switching speed, are much more tolerant of high temperatures and radiation and do not dissipate significant energy. Many applications especially where solid state devices fall short, prefer VME devices such as flat panel field emission displays (FEDs), electron microscopes, electron spectrometers, electron lithography systems, microelectronics devices, microwave devices, amplifiers for satellite communications, $\mathrm{x}$-ray sources and energy converters. ${ }^{1-4}$

Taking advantage of the advancement in top-down and bottom-up technologies, nanostructures with unique properties such as carbon nanotubes (CNTs), conical carbon nanotubes (CCNTs), metallic carbon nanotubes (M_CNTs), graphene and metallic nanowires, are investigated for possible cold and/or hot electron emitters. Generally, nanomaterials act differently when compared to bulk materials. For example, a nanostructure responds differently by producing EE when an electric field is applied. A macrostructure may not respond to the same excitation and needs a much higher field to 
produce cold emission. Conducting nanostructures are capable of delivering high energy electron beams with great precision at relatively low applied voltages. This chapter provides an introduction to the concepts of FE and TE of electrons from metals in general and in particular nanostructured materials. This chapter also addresses how nanostructured materials are integrated within experimental set-ups and how they can resolve most of the limitations facing the existing electron emission sources. Lastly, nanostructured emitters are compared with their existing semiconductor peers.

\section{1-1. Definition of EE}

The process of liberating free electrons into a vacuum from a solid surface caused by an external energy transferred to the electrons is termed as EE. EE is well known to occur from metals, because they have a sufficient amount of free electrons in between the atoms of their crystal lattice. Electrons in metals move freely through the bulk, but cannot leave the surface because of the presence of an electrostatic force holding them back to its core. Therefore, electrons see a potential barrier at the metal-vacuum interface. According to classical mechanics, in order for the free electrons to be emitted from the surface of metal, they require additional external energy at least equal to the potential barrier. Consequently, smaller barriers result in easier extraction of free electrons. However, quantum mechanics states that once a particle (i.e. electron) has enough energy (usually less than the work function), the potential barrier can be crossed with some finite tunneling probability producing free charges from the conducting solid into the vacuum level. This leakage current could be of some significance if the parameters governing its occurrence are understood and controlled. 
Most conducting materials emit electrons by several distinct mechanisms. For instance, CBNs such as carbon nanotubes (CNTs), carbon nanoclusters, graphene and diamond appear to be cold and hot emission candidates merely because low applied electric field and moderate temperature are enough to stimulate FE and TE of electron from this type of structure. Diamond emits electrons at the lowest applied field of any known material. In fact, it requires a negative electron affinity (NEA) to efficiently start EE. To obtain a NEA, one needs to introduce heavy p-type dopants within the crystal lattice of the diamond together with a monolayer of an electropositive material. Metallic thin film coatings (i.e. Cesium $(\mathrm{Cs})$ or $\mathrm{Cs} / \mathrm{O}$ ) are also used to induce NEA.,

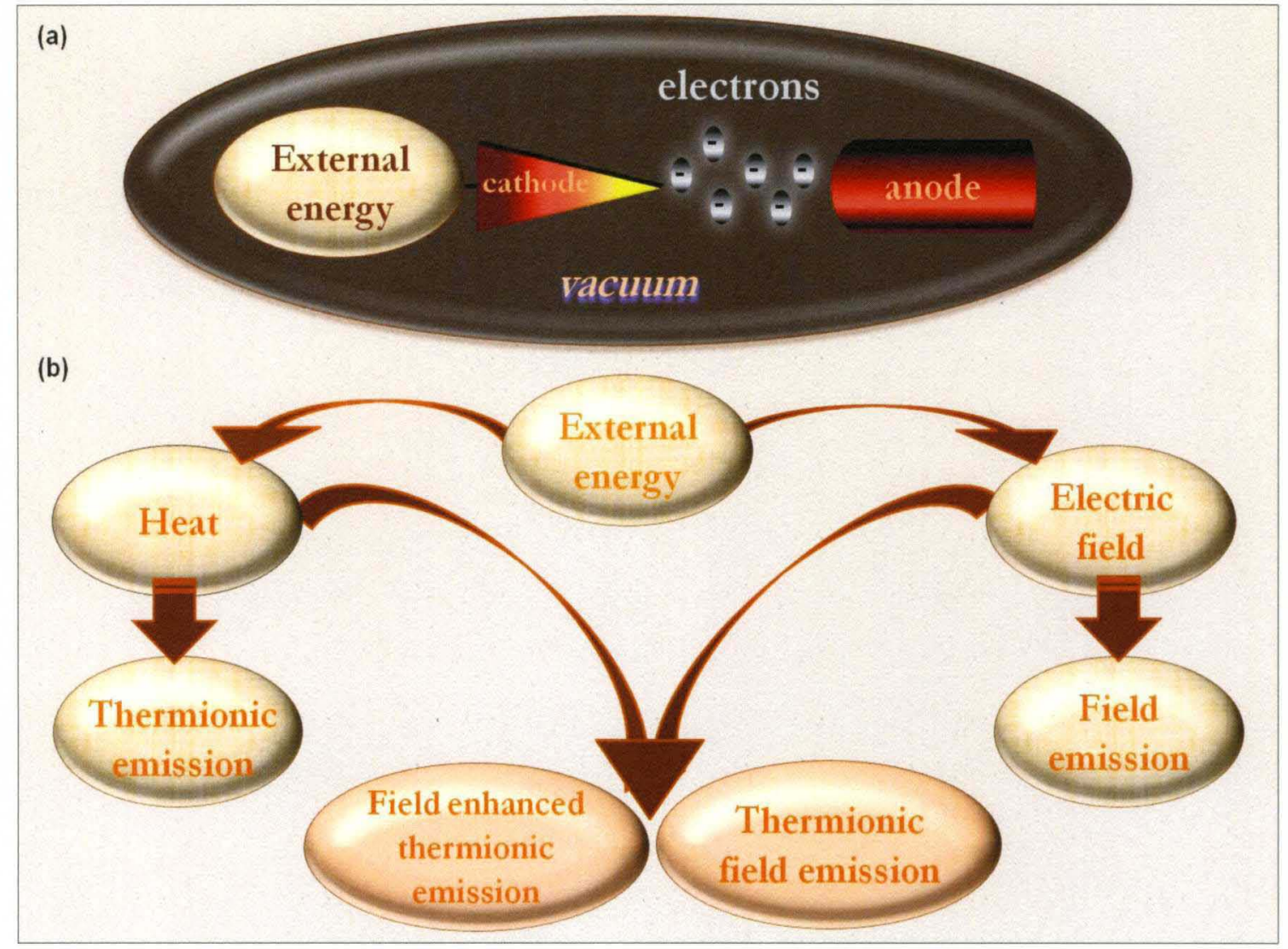

Figure 1- 1: Types of EE from nanostructures. 
Several forms of energy can be used to induce EE. These include heat, light, or electrostatic fields; accordingly, different types of EEs take place. Figure 1-1(a) is a schematic of EE from a tapered conductor caused by an external excitation. Figure 1-1(b) shows three different kinds of EEs that can take place once the solid is subjected to an external excitation.

A nearby anode having an opposite charge collects the emitted electrons. The entire system is placed within the vacuum (pressure greater than $10^{-7}$ Torr is desirable). ${ }^{2}$ Error! Reference source not found. The nature of the supplied external energy defines the type of EE from the system under test.

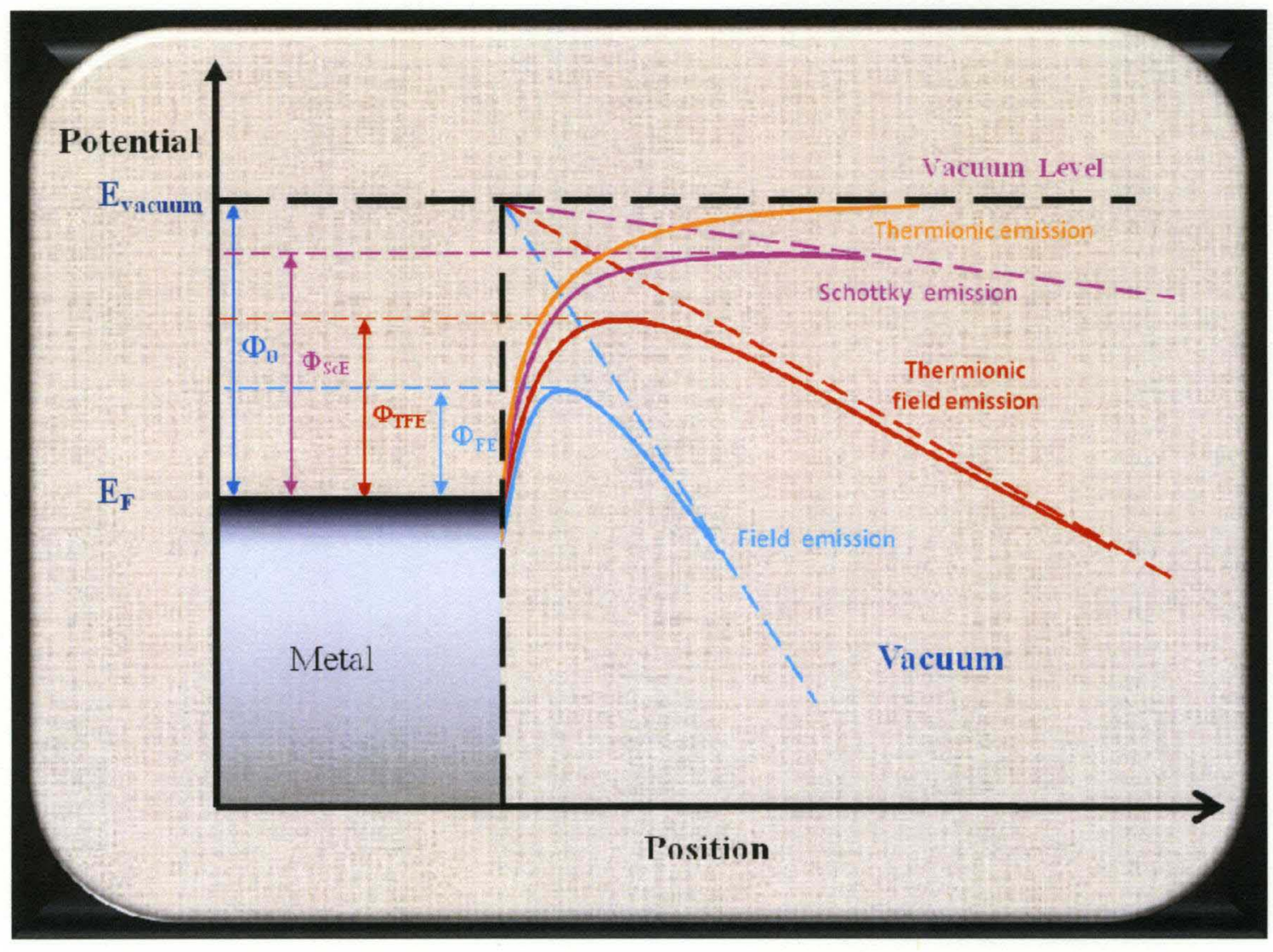

Figure 1-2: EE processes. 
Figure 1-2 illustrates the different processes of EE taking place within the metalvacuum interface once external excitations are present. ${ }^{7}$ The pair of electric field and temperature determines the predominant EE process that takes place at the metal-vacuum interface. On the other hand, the work function determines how easily the electrons can jump over the potential barrier. 


\subsubsection{Thermionic emission}

TE or hot emission occurs when the external energy supplied to the cathode is provided by heat. This emission is the process of liberating free electrons, at zero electric field, when the temperature of the bulk material increases to a value at which electrons gain sufficient energy to escape over the barrier height into the vacuum level. Most materials can emit electrons by the TE process if a suitable amount of heat is provided. Generally the heat corresponds to high input power and high temperature. Only few materials can emit at low temperatures and to achieve this they need to have a low work function; e.g. oxides.

Figure 1-3 is a schematic of the potential barrier for three different metals without the presence of an applied electric field. The barrier height that electrons see decreases as the work function decreases $\left(\Phi_{1}>\Phi_{2}>\Phi_{3}\right)$. The decrease can be observed when one treats the cathode's surface through coatings of electropositive materials. ${ }^{8}$ Treatment of the cathode using oxides significantly lowers its work function.

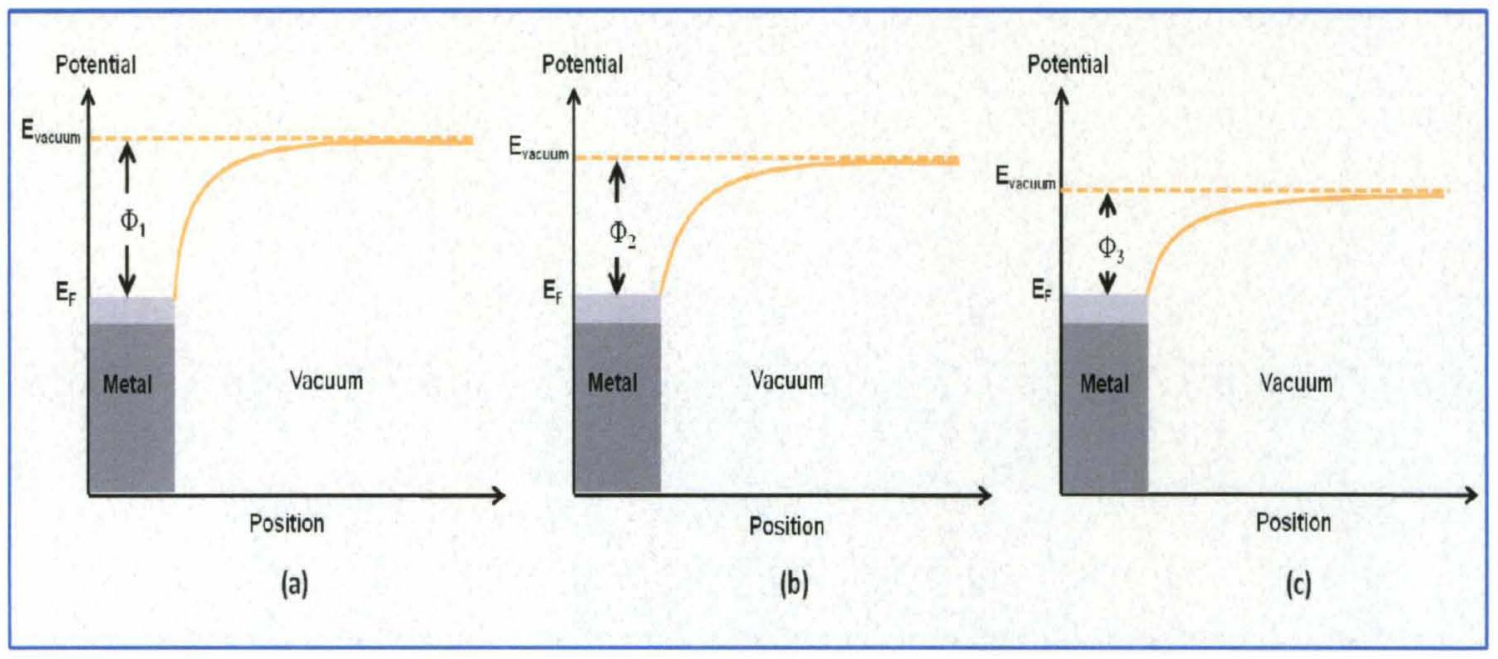

Figure 1-3: Potential barrier height for different metals. 
The magnitude of the charge flow increases significantly with increasing temperature, as the TE current has a quadratic dependence on temperature and an exponential dependence on its inverse. Equation 3 illustrates this dependence.

$$
I=c T^{2} e^{-\left(\frac{\Phi}{K_{b} T}\right)}
$$

Where $I$ is the TE current in $A, T$ is the temperature in $K, \Phi$ is the work function of the emitting material in $e V, K_{b}$ is the Boltzmann constant and $c$ is a constant. TE from pure metals becomes significant only for temperatures over $1500 \mathrm{~K}$ since their work function ranges between 4.5-5.10 eV. Equation 1-1 shows that TE is highly dependent on the cathode material through its work function. Most metals melt before they reach the onset temperatures of the emission (usually above $2000{ }^{\circ} \mathrm{C}$ ). The commonly used metal for TE is tungsten wire. Usually tungsten is classified as a direct-heated cathode. The tungsten wire is bended to form a tip with a small radius (hundreds of microns) of curvature. The tip-like structure starts to emit when its temperature increases by passing a high current through the wire. Therefore, materials having high work function and low melting temperature are not suitable for the TE. Space charge effect is another limit to TE at zero electric field. Because most metals are impractical to use as thermionic emitters and to overcome these limitations, people have used the oxide coating to lower the work function or search to create new materials and structures. Tungsten filaments are still employed because of their high melting temperature and ease of integration into tubes applications that operate with anode voltages greater than $20 \mathrm{KV}$.

As a consequence of these factors, there is a motivation to develop and characterize new materials capable of producing high and stable TE currents densities at 
relatively low temperatures. To obtain higher TE current densities, there is a need for low work function materials that have high melting temperature or lower the work function of a preexisting material. One of the techniques used to lower the work function is surface treatments or decoration where a thin layer of low work function materials like $\mathrm{LaB}_{6}$ or $\mathrm{ZrO}$ is deposited on a tungsten tip. The corresponding work function is $2.52 \mathrm{eV} .{ }^{9,10}$ Work functions of $1.1 \mathrm{eV}$ and $1.36 \mathrm{eV}$ is practically achievable by means of oxides coatings and adding cesium to metals (i.e. tungsten). ${ }^{11}$ Field Enhanced Thermionic Emission (FETE), known also by Schottky effect, is another way to lower the potential energy barrier. The application of an electric field bends down the potential barrier so that electrons need less energy to reach to the vacuum level. In this case, the field needs to be high enough for a possible significant barrier banding. Section (1-2) describes the effect of the field enhancement on the potential barrier lowering. Therefore using nanostructures can ease the barrier banding and produce better TE; however, the stronger the field the more likely is the thermionic field emission or pure FE to predominate the EE. Hence, the limit of the applied field is where the tunneling phenomena have a major contribution to the total emission current. In contrast; the potential lowering by thin film coatings is more significant. 


\subsubsection{Field emission}

When the external energy supplied to the system is electrostatic (an applied electric field exists between two electrodes), cold EE occurs. This emission is the tunneling of electrons, present at the Fermi level and having energy less than the barrier height, through the potential barrier which is deformed due to presence of high electric field as Figure 1-4. illustrates. ${ }^{12}$

Figure 1-4 is a schematic of the potential barrier existing within the metal with and without the presence of an applied electric field. The work functions $\Phi$ and $\Phi_{\text {eff }}$ are the energies required for an electron to surmount to get to the vacuum level. The strength of the electric field determines the shape of the potential barrier.

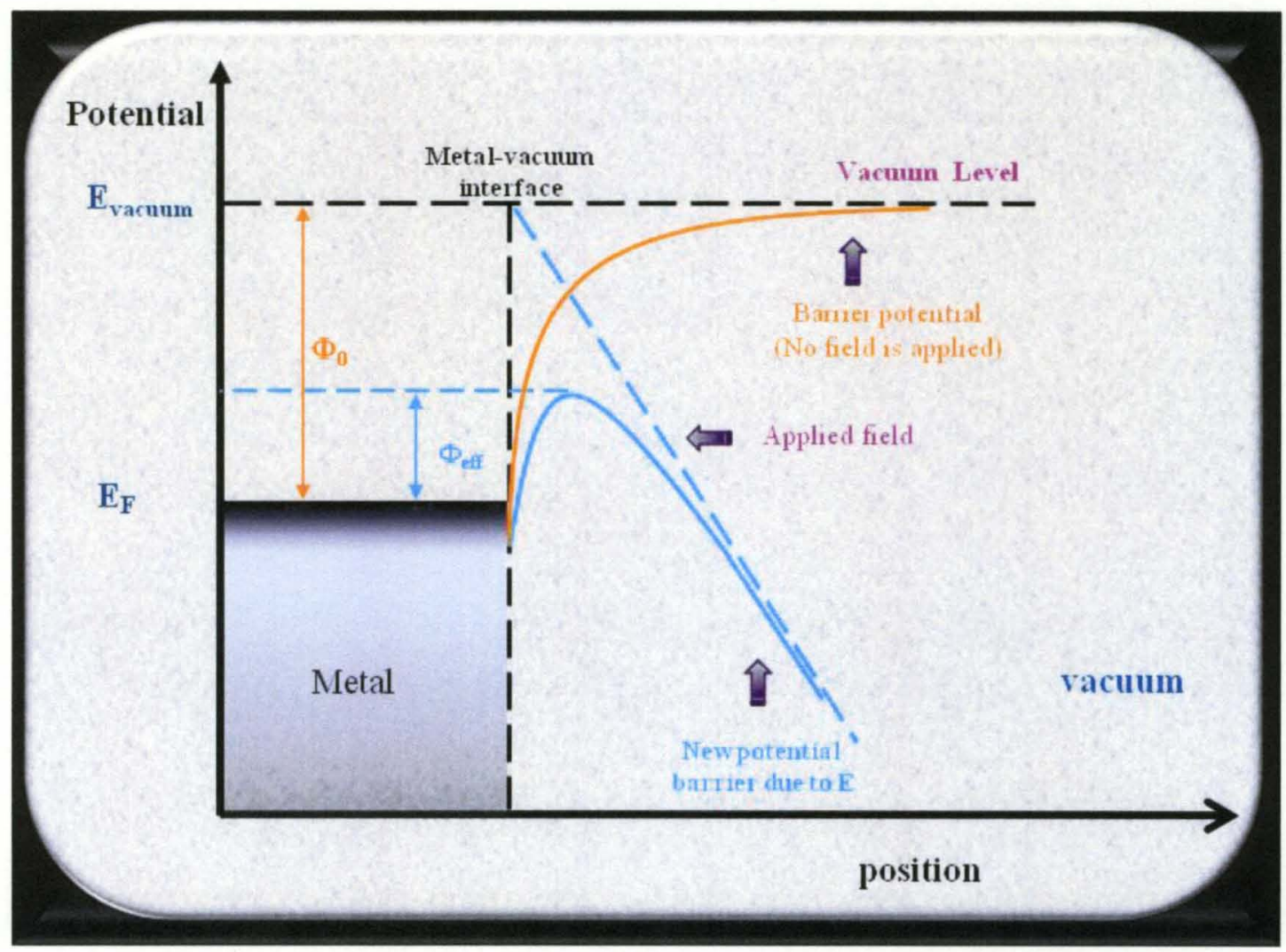

Figure 1-4: Schematic of EE from a metal. 
Top-down technology created VME which concerns itself with devices exploiting electron ballistic transport within the vacuum after emission from microstructures. One of the interesting aspects of FE is the nonlinear relationship between the applied voltage and emission current. For instance, within the emission regime, a small change in the input voltage results in significant change in the output current. Equation 1-2 shows the dependence of the emission current on the applied voltage.

$$
I=a V^{2} e^{-\left(\frac{b}{V}\right)}
$$

Where $I$ is the emission current in $A, V$ is the applied voltage, $a$ and $b$ are constants.

FE occurs at a very high electric field $(\mathrm{E}=\mathrm{V} / \mathrm{d})$, usually in the order of 1-10 $\mathrm{KVolts} / \mu \mathrm{m} .{ }^{12}$ This is regarded as a considerable input energy in order to extract electrons from ordinary metallic flat surfaces. Flat and smooth surfaces reveal a uniform field with no preferred region for EE. Surfaces having tip-like structures exhibit local field enhancement at these tips, hence turning into possible FE sites.

The field enhancement factor $\beta$ is used to quantify the amount of increase in the electric field due to amplification. $\beta$ is the ratio of the local field at the tip, $\mathrm{E}_{\mathrm{t}}$ to the applied field, $\mathrm{E}_{\mathrm{a}}$ at its base. Equation 1-3.

$$
\beta=\frac{E_{t}}{E_{a}}
$$


The field enhancement is a process of amplification of the applied electric field at the base of the tip by an order of up to a few hundred. Therefore, the use of sharp tips having sub-microns diameter causes a local field enhancement. Moreover, the sharper the emitter, the more intense the field is at its tip. Supersharp tip (diameter of 10-100 nm) magnifies the electric field by a few thousand.

The effect of field enhancement is visualized on the change within the shape of the potential barrier sketched on Figure 1-5 along the energy band diagram of a nanostructure. $^{12}$

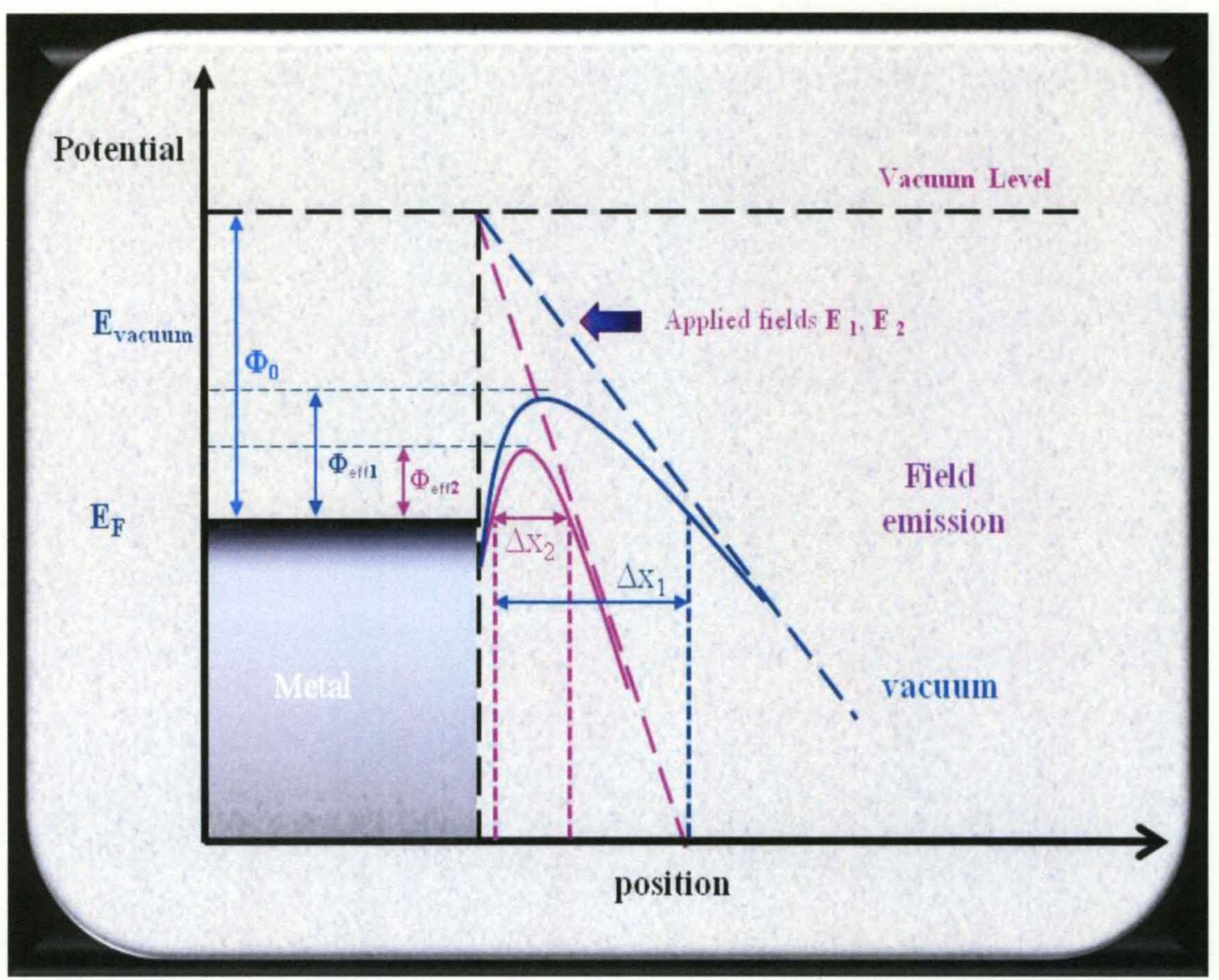

Figure 1-5: Field emission enhancement due to the use of sharp emitter. 
Field enhancement within the structure involves the lowering of the potential barrier and the decrease of the tunneling distance. The slope of the resultant potential barrier (blue curves) outside the metal is proportional to the electric field, and an observable shift on the new effective potential barrier towards the metal is produced (brown curves). In this case the field $E_{2}$ is greater than $E_{1}$.

Figure 1-5 is a schematic of the potential barriers formed as a result of applying two different electric fields to a single emitter. For a given cathode, once the electric field $E_{1}$ is applied, the effective potential (blue curve) bends near the metal and takes an asymptotic behavior to the resultant potential (blue line). Applying a higher field, $\mathrm{E}_{2}$, (purple curve) or using sharper emitter from the same material results in similar effects on the effective potential: it bends nearer towards the metal. Therefore, the tip's geometry enhances the local electric field and lowers the effective work function.

The shape of the potential barrier presented on Figure 1-5 is valid only for an individual cathode. In the presence of neighboring emitters, the curve bending may look different especially when the distance between the emitters gets small. In fact; it is shown that close packed arrays of CNTs are not ideal for FE applications as the close packing of the tubes screens the applied field effectively reducing the field enhancement of the high aspect ratio tubes. It is necessary to have individual vertically aligned tubes spaced apart by twice their height to minimize field shielding effects and to optimize emitted current density. ${ }^{13-15}$ 


\section{1-2. Vacuum Microelectronics}

\section{1-2-1. Definition}

VME of the fabrication and use of large area cathodes as EE sources based devices. In these devices, a high density of individual emitters (up to $10^{-7} \mathrm{emitter} / \mathrm{cm}^{2}$ ) is fabricated. The birth of nanotechnology and discoveries of nanomaterials emerged with VME to produce vacuum nanoelectronics (VNE). VNE employ nano-cathodes rather than micro-cathodes as the active emitting element.

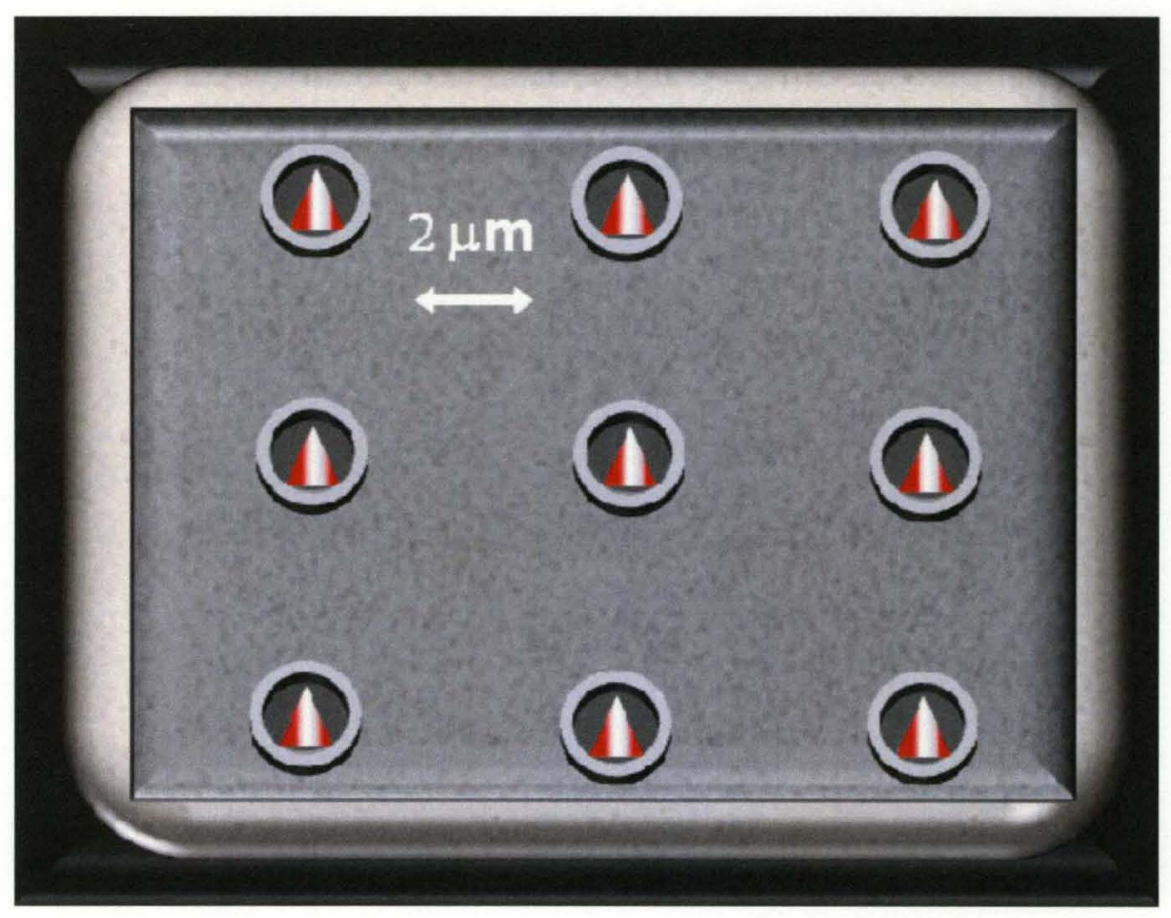

Figure 1-6: Field emission arrays.

Figure 1-6 is a schematic of FEAs where microfabrication techniques are used to make regular arrays in which cones are placed in small empty cylindrical spaces in an oxide film. A metallic coating is deposited on the oxide to make a counter electrode grid.

The field emission arrays (FEAs) (also referred to as Spindt arrays) are made of well spaced molybdenum cones. Each cone, because of their size and relatively high 
packing density (maximum of $10^{7}$ cones $/ \mathrm{cm}^{2}$ ), along with the presence of a gate (grid) surrounding each emitter, attracted most of attention and are the breakthrough of VME technology. ${ }^{8,16}$ FEAs use the microfabrication techniques adopted by silicon integrated circuit (IC) technology to make regular arrays in which molybdenum cones. Typically the active elements in VME systems are emitters with dimensions of tens to a few hundred of nanometers. The emitters are usually semiconductors, metallic or carbon based structures

\section{1-2-2. VME devices versus solide state devices (SSD)}

SSD allow high frequency operation, low power consumption, high fabrication yield and low production cost. Enormous progress in solid state electronics has been achieved and a lot of applications witness the extensive usage of solid state devices. However, there are still applications where solid state devices are inconvenient especially where tolerance to radiation and high temperature is desired since these devices usually use a single crystalline solid for the charge carrier mobility. In such applications, solid state devices need radiation shielding and thermal isolation to perform efficiently. On the other hand tolerance to radiation and heat are the main advantages of VME devices. In fact, temperatures of 200 ${ }^{\circ} \mathrm{C}$ and radiation exposure of $15 \mathrm{MRad}$ will not affect the functionality of FE cathodes. ${ }^{17}$

VME utilizes vacuum as the transporting medium. The carrier transport within this environment is ballistic, therefore, there is no energy dissipation through collisions. ${ }^{5,1,18}$ In this environments, radiation does not generate carrier charges, induce physical damage nor disturb the performance of FE devices. ${ }^{19}$ Resistance to high temperature allows nearer placement of the measuring devices to high temperature environments and a smaller need for heat sinks and other heat transfer accessories high power applications. 
In addition, there are other benefits of using vacuum medium, such as the lack of inertia and a very short electron transport time. As a result, high-frequency devices FE devices can be produced. The cutoff frequency can go up to $100-500 \mathrm{GHz} .^{18}$

Table 1-1 shows a comparison of some of the properties of VME devices with those of solid-state devices. ${ }^{18}$ It summarizes the main figures characterizing VME and solid-state devices.

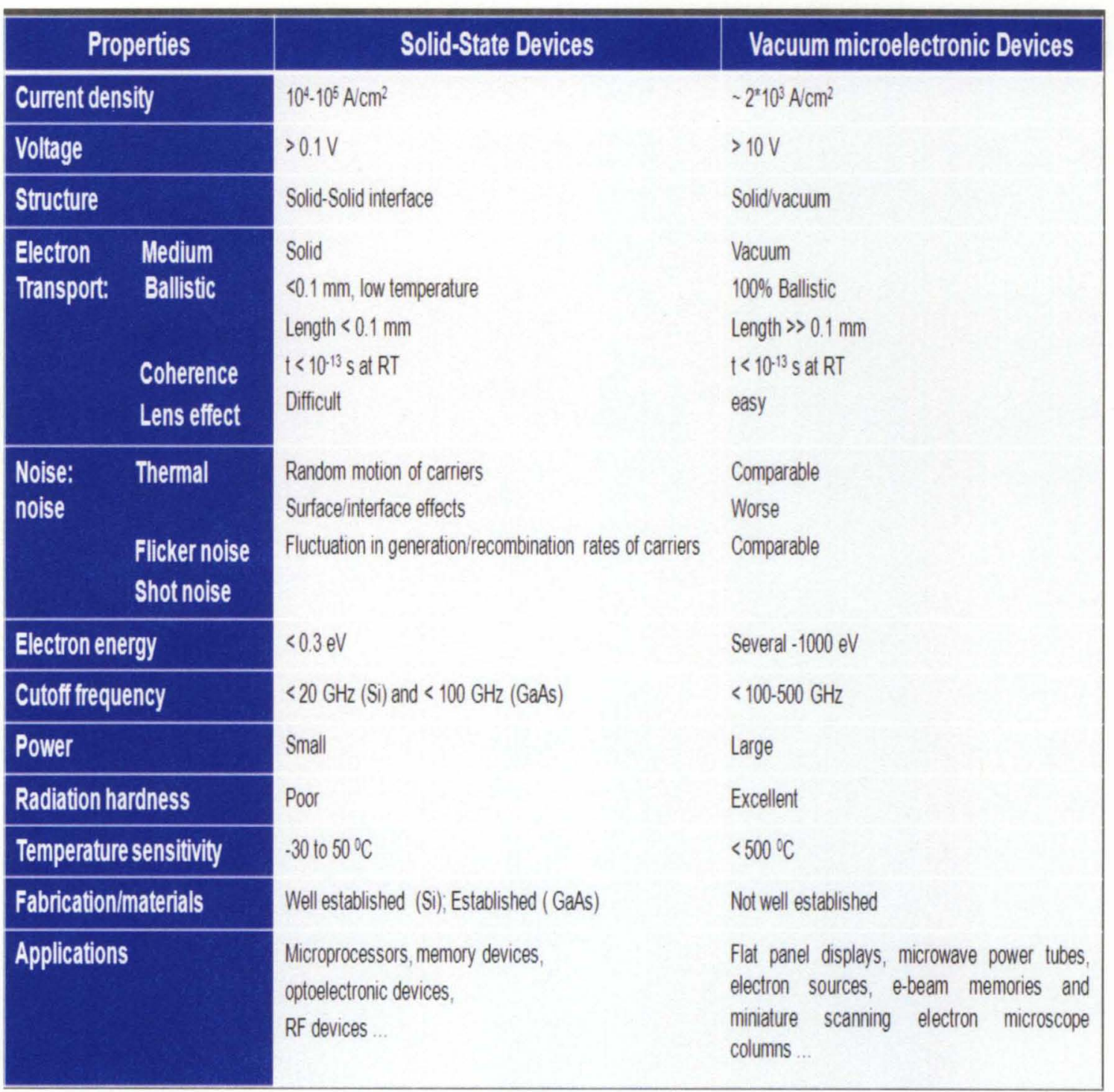

Table 1-1: Vacuum Microelectronic Devices versus. Solid-State Devices. ${ }^{18}$ 
According to table 1-1, the electron emitters are the active elements within many applications especially where solid state devices fall short. One application includes the development of improved flat panel field emission displays (FEDs) ${ }^{2}$ The other applications are ion sources used for electron optic systems such as electron microscopes along with electron spectrometers and electron lithography systems, microelectronics devices including diodes and triodes, microwave devices, amplifiers for satellite communications, x-ray sources and energy converters. Amplifiers can be used in microwave electronics. ${ }^{1-4}$

\section{1-2-3. VME versus vacuum nanoelectronics solutions}

Even though VME technology has experienced enormous growth and development, their emitters still face challenges: ${ }^{20}$

1) Low emission current density: Certain applications require current higher than $10^{5} \mathrm{~A} / \mathrm{cm}^{2}$ to be practical due to the limits on the shape and intrinsic properties of VME cathodes. $^{21}$ These cathodes usually have high work function, lower field enhancement and relatively lower packing density which reduce EE. In fact, the field enhancement factor of any existing microstructure emitters can't get larger than $10^{22}$ Therefore, the resulting turn-on and threshold electric fields are high while the emission current density is low. ${ }^{23}$

The ability to design new structures having new materials with nano-precision and better properties (i.e. higher field enhancement) reduces significantly (by few thousand) the operating voltage of the VNE devices. Moreover, the nano-emitters have better capability of sustaining higher emission current densities at relatively low threshold voltages. ${ }^{12}$ 
2) Low packing density: FEAs are fabricated based on photolithography techniques. Limitation on the minimum feature size to be patterned due to the limitation on the technology itself, limits the number of emitters to be fabricated in a $1 \mathrm{~cm}^{2}$ area. The maximum packing density is reported to be around $10^{7} \mathrm{tips} / \mathrm{cm}^{2}{ }^{8}$. This limits the emission current density. In theory, more nanostructures can be packed within $1 \mathrm{~cm}^{2}$ area than microstructures. A packing density of $10^{12} \mathrm{tips} / \mathrm{cm}^{2}$ is possible using nanostructures. ${ }^{1}$

3) High input energy: The higher the voltages required to turn-on and maintain (threshold voltage) a steady and stable EE, the more energy is consumed by the FE based devices. FE from nanostructures is routinely achieved at much lower turn-on and threshold fields $(0.4$ Volts $/ \mu \mathrm{m}$ and $10 \mathrm{Volts} / \mu \mathrm{m}$ are respectively reported for $\mathrm{CNTs})^{24}$

4) Emitter materials: The effective work function of a microfabricated tip is generally different from the bulk one because of the effect of the operating conditions on the emitting surface. Using materials with high work function elevate the operating voltage resulting in the chance of emitter's damages. ${ }^{18}$ Nanostructures produce local field enhancement at their tips resulting in lower operating voltages.

5) Emission lifetime and brightness: The short cathode's lifetime is attributed primarily to the sensitivity to impurity adsorption (contamination), surface chemistry (the willingness of the emitters to react with the substrate), probable variation in its work function, ion bombardment, sputtering and poisoning. ${ }^{18,25}$ In case of hot emission, brightness increases with temperature, while lifetime decreases significantly with the increase in the operating temperature. For example, tungsten filaments, used as electron 
sources within microscopes, gain about $70 \%$ increase in its lifetime when the operating temperature drops from $2800 \mathrm{~K}$ to $2700 \mathrm{~K}^{26}$

Carbon nanotubes, as an example, have a smaller chance to react, get contaminated and affected by the vacuum environment simply due to their superior properties. $^{27-29}$ Moreover, since the VNE devices use nanostructures as their active element, the emission sites are much brighter than the VME devices. ${ }^{30}$

6) Emission stability: The emission instability is due mainly to the emitters' non-uniformity, defect and wear. It is difficult to microfabricate cathodes that have precisely similar height and shape. The emission current is very sensitive to the change in the local electric field. In fact, the current is very sensitive to the shape, topology, surface area, and local work function of the cathode. Indeed, the FE current varies over many orders of magnitude for a small variation in the radius of the emitter. ${ }^{23}$

Nanostructures can be routinely fabricated with high uniformity and mechanical strength (i.e. carbon nanotubes and nanowires) resulting in better emission stability.

7) Emission current fluctuation noises caused by two different types of short term current fluctuations are detected while collecting emitted electrons of single tip Spindt-type microfabricated cathodes: shot noise for frequencies greater than $100 \mathrm{kHz}$, and bistable noise, generated by adsorbates switching between emission states, for lower frequencies $(10-100 \mathrm{KHz})$ at temperatures smaller than or equal to $300 \mathrm{~K}^{29,31}$

8) Fabrication processes are expensive as critical processes involve the cleanroom environment and optical or e-beam lithography. The progress observed in nanomaterials synthesis especially in the growth of nanomaterials using the bottom-up technique; therefore, require much less complicated techniques and technologies to 
produce nanostructures. Technically, self-assembly can be the basis of the creation of field emission arrays.

9) Joule heating of the cathode: Thermal runaway caused by joule heating may cause distortion or deformation of the emitters when high current passes through. CNTs are an example of nanostructures that sustain high current, since their melting temperature is $4800 \mathrm{~K}$.

Implementing nanomaterials within VME devices can improve the emission reliability and create new applications such as portable X-ray sources. This is credited to the fast development and exploitation of nanotechnology and nanostructures. 


\section{1-2-4. Nanoscience and nanomaterials for better VME devices}

Nanoscience and nanotechnology has grown rapidly in the last decade. This progress is seen in the synthesis, fabrication and assembly of nanomaterials. In addition, new characterization techniques, device fabrication and technologies have been launched. Discovery and growth of new nanomaterials is continuously reported - including nanowires, carbon nanotubes, graphene and diamond. Because of the increasing availability of methods of synthesis of nanomaterials as well as tools of characterization and manipulation, novel methods of fabrication and control of nanostructures, and new device concepts are being constantly discovered.

Nanotechnology deals primarily with the exploration of nanostructures Such as clusters, quantum dots, nanocrystals, metallic nanowires diamond and carbon nanotubes and so on. ${ }^{32}$ The physical and chemical properties of nanomaterials can differ significantly from those of the bulk materials of the same composition. The uniqueness of the mechanical, electrical, chemical and thermal characteristics of nanostructures constitutes the basis of nanoscience and the reason behind their exploration as EE sources.

There are two approaches to nanoscience graphically termed 'top down' and 'bottom up' or self-assembly. 'Top down' refers to making nanoscale structures by machining and etching techniques, whereas 'bottom up', or molecular nanotechnology, applies to building organic and inorganic structures atom-by-atom, or molecule-bymolecule. Both methods have evolved separately and once combined with suitable control of the properties and response of nanostructures will lead to new devices and technologies. 
Well-established technologies are based on nanomaterials such as SPM, SEM, and nanoelectronics devices. However, the goal of science and engineering of nanomaterials technology is to master the synthesis and fabrication in order to explore and establish nanodevice concepts, to generate new classes of high performance nanomaterials and to improve the techniques of nanostructures investigation. Potential uses of nanomaterials includes but is not limited to nanoelectronics, nanofluidics, nanooptics, nanomagnetics, and nanobioelectronics. ${ }^{33}$

Nanomaterials, especially CBNs, were able to emerge within the VME to develop vacuum nanoelectronics technology (VNE). The essential elements of vacuum nanoelectronics devices are microfabricated or synthesized single nanoemitters. Results have been achieved while exploring nanomaterials as electrons emitters. ${ }^{34}$ For instance, the core of most of the current electron guns are based on nanoemitters capable of producing focusable small spot size beam. ${ }^{35}$

VME devices based on microfabricated arrays reached its limit for the packing density $\left(10^{7}\right.$ cones $\left./ \mathrm{cm}^{2}\right)$ because of the limitation in photolithography. ${ }^{8}$ Since the maximum number of emitting sites is limited, the extracted current density also has a maximum. A solution for this issue consists of using a bottom-up approach and by switching to nanostructures. The published values of turn-on fields from VME emitters are greater than $8 \mathrm{~V} / \mu \mathrm{m} .{ }^{36-38}$ Using CBNs based devices this number can be reduced by at least one order of magnitude. In the present work, a turn-on of $0.7 \mathrm{~V} / \mu \mathrm{m}$ is obtained from conical carbon nanopipettes.

Constructing a practical VME field emitter device necessitates uniform tip height and diameter (50-100nm). VME emitters are costly to make because their fabrication 
requires the use of MEMS procedures involving the photolithography technique. Using nanostructured materials as EE sources is a promising route towards the production emitters having high uniformity in the shape, emitting surface area and height. This is possible by the bottom-up approach and the ability to grow CBNs with the requirements of high uniformity in diameter, height and other properties.

Firstly, CBNs, especially carbon nanotubes, are known to have good electronic and mechanical properties and proven to be extremely stiff and resistant to bending, ion bombardment and harsh vacuum environment. ${ }^{27,28,39-41}$ Secondly, the good thermal properties of CBNs can be seen in their high thermal conductivity $(\sim 6600 \mathrm{~W} / \mathrm{m} \mathrm{K})$ and melting temperature of carbon around $4800 \mathrm{~K} .^{42,43}$ It was reported that SWCNTs are thermally stable in vacuum under temperatures as high as $2800{ }^{\circ} \mathrm{C}^{44}$

These advantages over metals and semiconductors emitters make CBNs stand firm to the harsh environments including the joule heating. Finally, the chemical properties of CBNs are outstanding and are unreactive with the substrates. Having all of these characteristics it is highly possible that the fluctuation noise within the noise spectrum can be reduced. ${ }^{18}$ Carbon based materials are known to have high work function ranging 4.5-5.10 eV. Recent work shows that a simple oxide coating can significantly reduce the work function to $1.9 \mathrm{eV} .^{45}$ Beside all of this, the growth processes of most nanostructures and $\mathrm{CBNs}$ is inexpensive compared to the need for sophisticated equipments used in the VME processes. 


\section{1-3. Dissertation outline}

Chapter 1 is a brief overview of the different processes of emission that occur at the metal-vacuum interface. The advantages of using nanostructured materials as electron point sources over the pre-existing emitters have also been explained.

Chapter 2 discusses the theoretical backgrounds of the FE, TE, and Field enhanced TE. The theory will be used to understand and to analyze the experimental data presented in chapter 4 . Chapter 2 also presents the material selection and the design needed to optimize EE properties. Modeling of emission current density is introduced together with the estimations and validity regions for each class of EE. In addition, a literature review of the commonly used TE materials is presented.

Chapter 3 presents the fabrication and synthesis procedures used to create nanoemitters used in this study plus a brief overview of the growth process for the existing ones. The experimental set-ups and requirements employed for bulk in addition to In-situ characterization, and set-up optimization is presented.

Chapter 4 discusses the experimental results and analysis of TE characterization from carbon based nanostructures. The techniques used to extract practical parameters of nanoemitters are presented.

Chapter 5 discusses the experimental results and analysis of FE characterization from different nanostructures. The techniques used to extract practical parameters of nanoemitters are demonstrated. Evaluation of the efficiency of the nanostructures used is established.

Chapter 6 presents summary and conclusions of the present work. 


\section{CHAPTER II}

\section{THEORETICAL BACKGROUND AND ESTIMATIONS}

This chapter reviews the theory of the three types of EE (FE, TE and FETE) to understand how an EE based device works and acts as a guideline to design and analyze the functionality of EE of nanostructured materials.

EE can be classified into three regimes based on the contribution of each of temperature and electric field to extract electrons from solids. ${ }^{46}$ Low electric field and high temperature produce thermionic emission. Under high field strength and low temperature, field emission prevails. Thermionic field emission takes place when tunneling phenomena along with thermal excitation are observed simultaneously at moderate field and temperature. During these regimes, the electron transport mechanism takes place within the vacuum medium in two different ways: electrons climbing over the barrier once they gain energy or penetrating through the potential when its height and shape permits. The resulting emission properties depend on temperature, field strength and materials' electronic properties from which electrons are to be extracted into the vacuum level. 


\section{2-1 Thermionic emission}

\section{2-2-1. Physics of thermionic emission}

TE is a temperature induced flow of charges from a charged solid when they gain thermal energy capable of overcoming the electrostatic forces holding them back to the solid (conductor). Figure 2-1 is a schematic of the potential barrier seen by an electron present within the conductor.

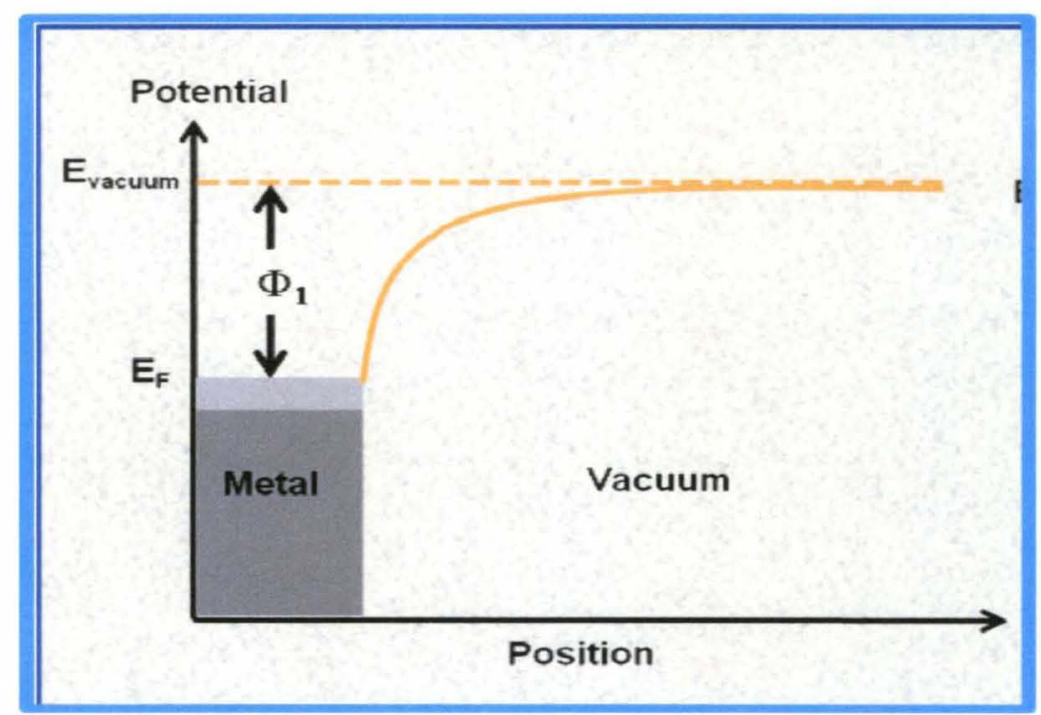

Figure 2-1: Schematic of the potential barrier diagram within metals.

Metals are materials capable of conducting electric current due to the presence of mobile electrons in the valence band. Electrons in the bulk of a conductor experience an average electric field that is zero.

The valence electrons, due the image charge effect, experience strong binding force, $F_{x}$, holding them within the conductor: ${ }^{47}$

$$
F_{x}=\frac{-e^{2}}{4 \pi \varepsilon_{0}(2 x)^{2}}
$$


Where $x$ is the distance of an electron from the surface of the conductor and $\varepsilon_{o}$, is the permittivity of free space.

In metals, the electrons are governed by Fermi-Dirac statistics, which states that the probability that a specific electronic state at energy $E$ is occupied by an electron in thermal equilibrium is given by the Fermi-Dirac distribution: ${ }^{48}$

$$
f(E)=\frac{1}{1+e^{\left(E-E_{F}\right) / K T}} \quad 2-2
$$

Fermi energy can be imagined as a distinction between the states that are occupied and those unoccupied (Figure 2-2). At absolute zero, the distribution function has a step (rectangular) at the Fermi energy and all the electrons occupy states at or below this energy. This transition smoothes out as the temperature is increased and electrons start filling up states higher than Fermi energy, furthermore, the distribution function for the electrons develops a high energy tail as depicted in Figure 2-2.

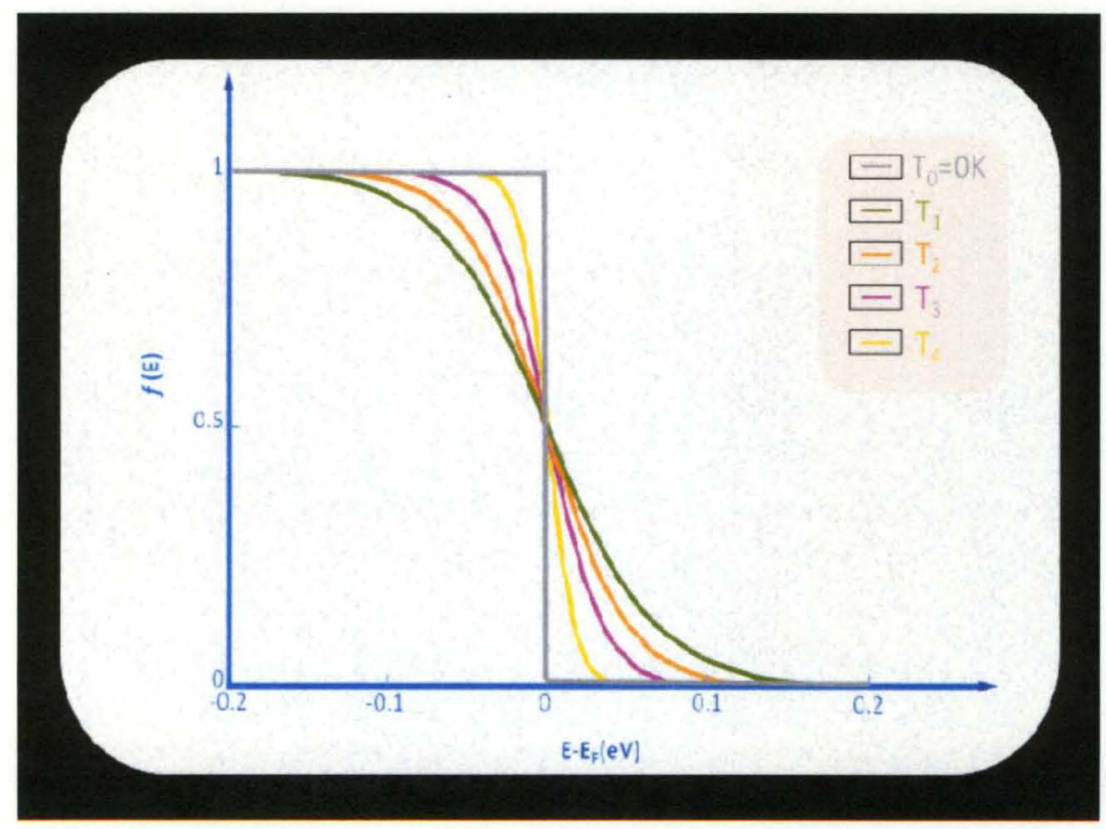

Figure 2- 2: The Fermi-Dirac distribution function at different temperatures. 
Figure 2-2 shows curves of the Fermi-Dirac distribution function $f(E)$ versus the energy $\left(E-E_{F}\right)$ for different temperatures. It illustrates the behavior of the distribution function as the temperature changes. At the Fermi energy the statistics require that half of available energy states will be occupied by an electron independently of temperature.

Once the temperature of a given material increases, electrons start vibrating, moving faster so that their kinetic energy increases. When the energy is high enough to overcome the image-charge force, the electrons are able to escape from the conductor's surface into the vacuum.. ${ }^{49}$ In this processes of electron "evaporation", the higher the temperature the larger the current of escaping electrons. Therefore, the rate of EE is related to the temperature.

The higher the electrostatic potential barrier (work function in this case) the more energy is required by electrons to pass over it. Therefore; the rate at which EE occurs must be related to the work function. The number of electrons escaping from the metal corresponds to an electric current, and Richardson's Law based on classical mechanics, states that the emitted current $I$ is a function of temperature $T$ via the equation: ${ }^{50}$

$$
I_{0}=A T^{2} e^{-\left(\frac{\phi}{K_{b} T}\right)}
$$

And in term of current density

$$
J_{0}=A T^{2} e^{-\left(\frac{\Phi}{K_{h} T}\right)}
$$

While:

$$
A=\frac{4 \pi m k^{2} e}{h^{3}}
$$


Where $T$ is the absolute temperature in Kelvin, $\Phi$ is the emitting material's work function in $e V$ at $O K, K_{b}$ is Boltzmann constant, $A$ is Richardson's constant, $A^{\prime}=A / S, S$ is the emitting surface area, $m$ and $e$ are the mass and charge of an electron and $h$ is Planck constant.

\section{2-2-2. Effect of the work function on thermionic emission}

The work function $\Phi$ is a characteristic of the emitting surface. In the field of TE, search for low work function materials has attracted lot of focus. Experimental evidence indicates that the $\Phi$ depends slightly on temperature, due to thermal expansion of the atoms lattice, through the coefficient $\boldsymbol{a}$ and, per se it can be expressed as: ${ }^{51}$

$$
\Phi=\Phi_{0}+\alpha T
$$

Therefore, the emission current density can be rewritten as:

$$
\begin{array}{ll}
J_{0}=A^{\prime} T^{2} e^{-\left(\frac{\Phi_{0}}{K_{b} T}\right)} & 2-7 \\
A^{\prime}=A e^{\alpha e / k_{b}} & 2-8
\end{array}
$$

Equation (2-4) shows the quadratic dependence of the emission current on temperature and exponential dependence on work function indicated by Equation 2-4. The quadratic dependence is shown in the individual curves in Figure 2-2. There is a large increase in the emission current with little temperature rise for $0.5 \mathrm{eV}$ change in the work function. Fitting TE experimental data into Equation 2-4, one can measure the work function of the emitting materials. ${ }^{52}$ The exponential dependence is on the work 
function. The slopes of the curves of Figure 2-2 are due to the difference in the work functions.

The effect of work function on emission current as well as onset emission temperature is evident. The value of the effective work function provides an insight on the operating interval of a TE based device and estimates of the emission current density. Figure 2-3 displays the emission current density versus temperature illustrating the effect of the work function on the emission properties.

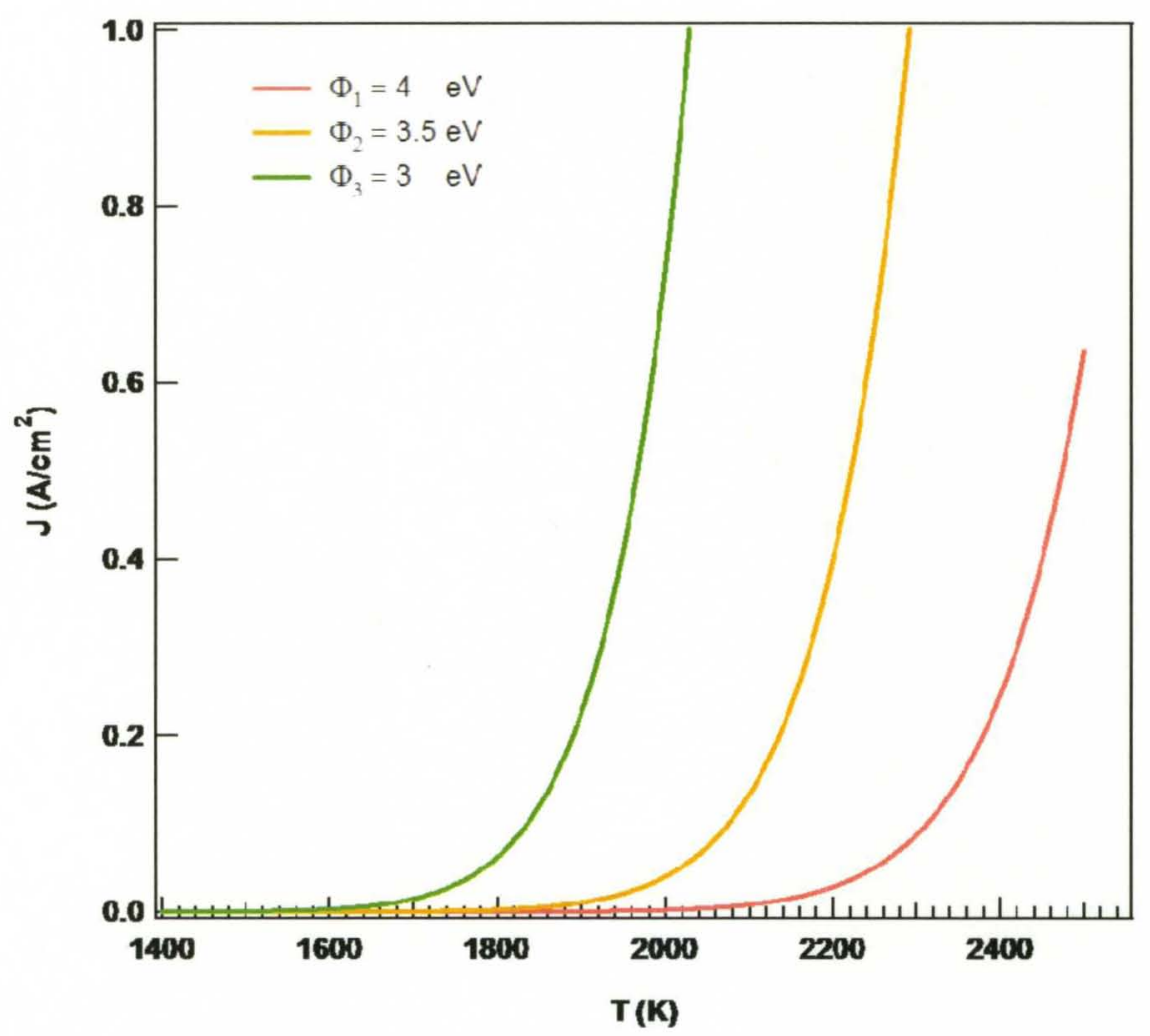

Figure 2- 3: The emission current density versus temperature for three different materials. ${ }^{53}$

While most of the emitting materials have work function ranging between 1 and $5.5 \mathrm{eV}$, very few can meet the requirement of low work function and high melting 
temperature. In fact, some people take the ratio of work function to the melting temperature as a figure of merit for thermionic emitters. ${ }^{54}$

A band energy diagram can also be used to illustrate how the work function of the material affects emission probability. Figure 2-4 shows a schematic of one dimensional energy band diagram in the absence of electric fields of three materials having different work functions. The smaller the work function the lower is the energy needed for the electrons to surmount the energy barrier. Any electron having energy less than the work function will not manage to escape but will be turned around by the intrinsic electric field close to the surface and eventually return into the body of the metal. Cathodes with low work function are well suited as thermionic emitters.

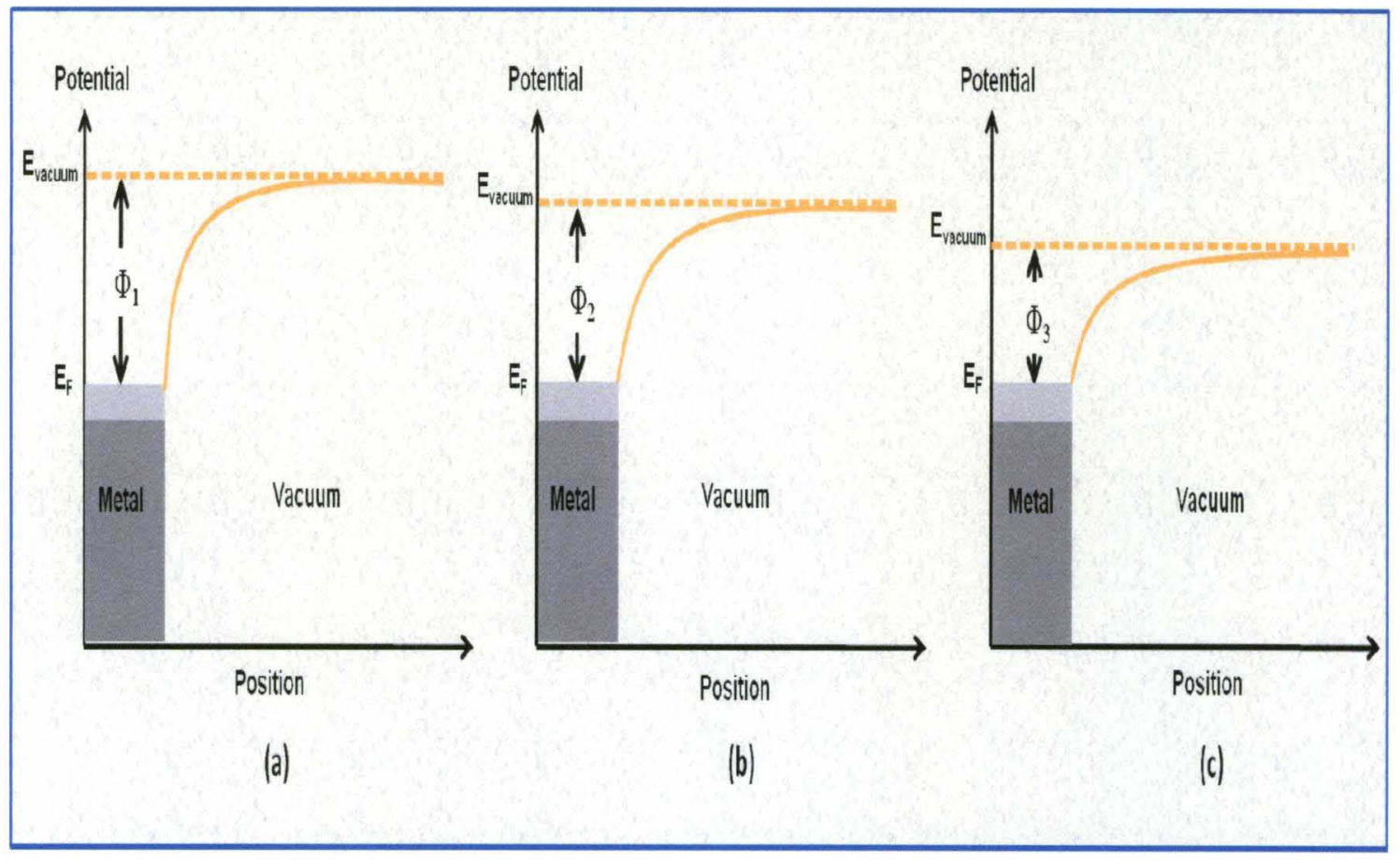

Figure 2- 4: The Energy band diagram of three different materials. 
Table 2-1: Effect of work function on thermionic emission properties

\begin{tabular}{|c|c|c|}
\hline Material & $\Phi(\mathrm{eV})$ & $\mathrm{T}(\mathrm{K})^{(*)}$ \\
\hline Tungsten $(\mathrm{W})$ & 4.6 & 2608 \\
\hline Thoriated Tungsten $(\mathrm{Th}-\mathrm{W})$ & 3.2 & 1870 \\
\hline Oxide & 1.5 & 942 \\
\hline
\end{tabular}

${ }^{(*)}$ Emission temperature needed to produce $1 \mathrm{~A} / \mathrm{cm}^{2}$.

Table 2-1 illustrates the TE properties of tungsten, thoriated tungsten and oxide that have different work functions. The effect of work function on reducing the TE onset temperature as well as increasing the emission current density is evident. For about $47 \%$ drop in the work function, the temperature required to obtain an emission current density of $1 \mathrm{~A} / \mathrm{cm}^{2}$ drops by a factor of 2 . 


\section{2-2 Field emission}

Field emission (FE) is the penetration or tunnelling of electrons from the surface of a conductor into vacuum under the influence of an electric field. Typical fields can be of thousands of Volts/microns. In order to obtain such field values at low applied voltages, the emitters must have a sharp tip at their ends. FE can be obtained at temperatures much lower than those required for thermionic emission and most of times at zero temperature; sometimes called cold emission.

FE requires higher vacuum levels than TE to be able to work with high efficiency. Unlike TE, FE doesn't require the electrons to have energies equal or higher than the potential barrier and low work function. In the FE process, the emitted electrons are produced from a cold surface rather than hot surface. Consequently, The FE devices consume less power and can be turned-on instantaneously. Fowler, Nordheim and others explained this phenomenon on the basis of quantum mechanical tunneling. ${ }^{55,56}$ In thermal equilibrium, electrons are confined within the conductor by a potential well and have energy insufficient to escape to the vacuum. Application of an external electric field to a conductor results in the bending and thinning of the surface potential barrier at the metalvacuum interface. ${ }^{56}$ Once the barrier lowers and the tunnelling distance becomes small enough, electrons penetrate through with finite probability. Figure 2-5 is a schematic of a one dimensional energy diagram seen by an electron on a metal surface. The potential barriers sketched are formed as a result of image charge in the absence or presence of electric fields. At large enough field the energy barrier can be narrowed enough to sustain large tunneling current. ${ }^{55}$ 


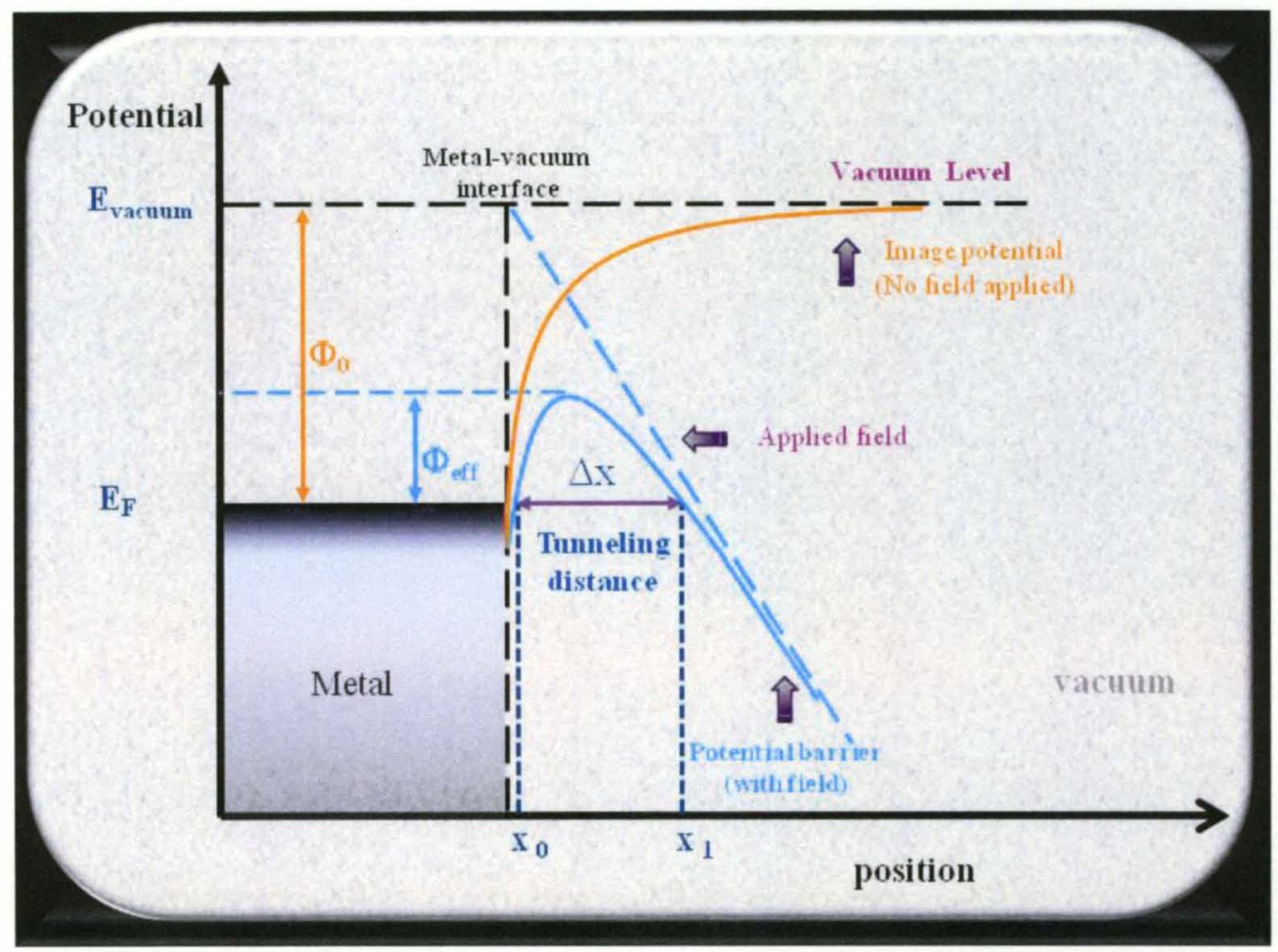

Figure 2-5: Energy band diagram showing the metal-vacuum interface and the potential barrier with and without the presence of an applied field.

The following expression describes the shape of the barrier drawn on figure 1.in the presence of an external electric field:

$$
V_{x}=E_{F}+\Phi-\frac{e^{2}}{4 x}-e E x
$$

Where $-e 2 / 4 x$ is the potential energy due to the image charge potential and $-e E x$ is the potential energy due to the applied electric field.

Figure 2-5 shows that the resultant barrier potential changes due to application of an electrical field. Both the barrier height is reduced and its peak has defined value so that tunneling probability becomes higher. FE is becomes easy to realize when the barrier width is less or equal to $1 \mathrm{~nm} .^{57}$ The barrier width (also called tunneling distance) $\Delta x$ can 
be computed from Equation (2-9). The intersection of the function $V_{x}$ with the horizontal line at the Fermi energy correspond to two x-coordinates $x_{0}$ and $x_{l}$, therefore the tunneling distance is defined as:

$$
\Delta x=x_{1}-x_{0} \approx \frac{\Phi}{e E} \quad 2-10
$$

From equation (2-10) shows that for given work function, application of high applied electric fields can significantly reduce the tunneling distance.

The resulting barrier height denoted as the effective work function $\left(\Phi_{\text {eff }}\right)$ can be expressed using equation (2-9) as:

$$
\Phi_{e f f}=\Phi-e \sqrt{\frac{e E}{4 \pi \varepsilon_{0}}} \quad 2-11
$$

Fowler-Nordheim (F-N) theory assumes that the metal obeys the free electron approximation with Fermi-Dirac statistic, the emitting surface is planar, smooth and having temperature of $0 \mathrm{~K}$.

The free electron model suggests that the emission current density is the product of the supply function or number of electron impinging on the potential barrier and the probability of the electrons that could penetrate through the barrier. ${ }^{56}$

$$
J(E, T, \Phi)=e \int_{0}^{\infty} D(E, W) N(T, \Phi, W) d W \quad 2-12
$$

Upon integrating Equation 2-12, the emission current density can be predict using F-N equation. ${ }^{58}$ 


$$
\begin{aligned}
& J=\frac{A}{t(y)^{2}} \Phi^{-1} E_{t}^{2} e^{\left(-\frac{B \Phi^{3 / 2}}{E_{t}} v(y)\right)} \\
& y=\frac{1}{\Phi}\left(\frac{e^{3} E_{t}}{4 \pi \varepsilon_{0}}\right)^{1 / 2}
\end{aligned}
$$

Where $e$ is the elementary charge of the electron, $J$ : current density in $\mathrm{Amps} / \mathrm{cm}^{2}$, $E_{i}$ : the effective electric field in volts/cm, $T$ is the temperature in $K, \Phi$ is Work function in $\mathrm{eV}, A=1.56 \times 10^{-6} \mathrm{~A} V^{2} \mathrm{eV}, B=6.83 \times 10^{7} \mathrm{~V}(\mathrm{eV})^{-3 / 2} \mathrm{~cm}^{-1}, S$ is the tip's emitting area in $\mathrm{cm}^{-1}, D$ is the penetration probability, $N$ is Number of electrons, $W$ is energy and $\beta$ is the field enhancement factor. The Nordheim elliptic functions $t^{2}(y)=1.1$ and $v(y)=0.95-y^{2}$ are slow varying on $E$ and $\Phi$. They have been computed, tabulated and for simplicity they are taken equal to unity in this work.

It can be concluded from Equation 2-13 that if $\ln \left(J / E^{2}\right)$ versus $1 / E$ is plotted, it should result in a straight line for a typical field emission experiment. $\beta$ can be calculated for a given emitting material using the computed value for the slope of the line. The electric field in equation (2-13) denotes the local electric field experienced by the emitting surface. In case of tip-like structure the applied and local electric fields are different and proportional via. ${ }^{59}$

$$
\beta=\frac{E_{t}}{E_{a}}
$$

Substituting equation (2-15) into equation (2-14), the current density expression becomes $\beta$ dependent: ${ }^{60}$ 


$$
J=A \Phi^{-1} \beta^{2} E_{a}{ }^{2} e^{\left(-\frac{B \Phi^{3 / 2}}{\beta E_{a}}\right)}
$$

The modified F-N equation above is to reflect the surface topography on field emission. The dimensionless parameter $\beta$ is then used is to quantify the ability of the emitter to enhance the applied electric field and to illustrate the cathode's performance. This enhancement manifests in the narrowing of the tunneling distance and reduction of barrier height (that's why it is called field enhancement factor) and is basically evaluated by the cathode's sharpness and aspect ratio. Since FE characteristics are determined by the local field $E$, then the higher the $\beta$ value of the emitter the lower the value of applied field at which significant emission takes place. Depending on the shape of the substrate and emitter, different empirical expressions for $\beta$, have common dependence on the aspect ratio $(h / r)$ of the emitter. ${ }^{61}$ While using flat cathodes with smooth surfaces the applied and effective (local at the emitting surface) electric fields should be the comparable. The applied electric field also needs to be high enough $\left(\sim 3^{*} 10^{7}\right.$ Volts $\left./ \mathrm{cm}\right)$ to turn on field emission and obtain meaningful current. However, when using nanostructures and rough surfaces having tip-like structures, it is unnecessary to have large field inputs. Nanostructures have the ability to amplify the electric field and since the local electric field can't be measured directly; the factor $\beta$ (which can be extrapolated using the F-N plot) is usually used to compute the local field and predict the occurrence of FE. Figure 2-6 illustrates that a flat and smooth surfaces exhibit a uniform low field while pointed structures shows high field surrounding the apex and local field enhancement. 
The value of the applied electric field at which field emission kicks off is called turn-on field. It is an indicative of the performance of the emitter. The following example illustrates the importance of local enhancement on some of the field emission properties. Low turn-on fields at which meaningful emission currents, usually corresponding o $\ln \mathrm{A}$ or $10 \mu \mathrm{A} / \mathrm{cm}^{2}$, can be extracted is very desirable.

As a first approximation $\beta$ can be approximated to the ratio of $h / r$. For an emitter having $5 \mathrm{um}$ in length and $20 \mathrm{~nm}$ in diameter, $\beta$ is approximately 500 . Therefore to reach required field emission field of 3000 Volts/um, just 6 Volts/um needs to be applied at the base of the emitter.

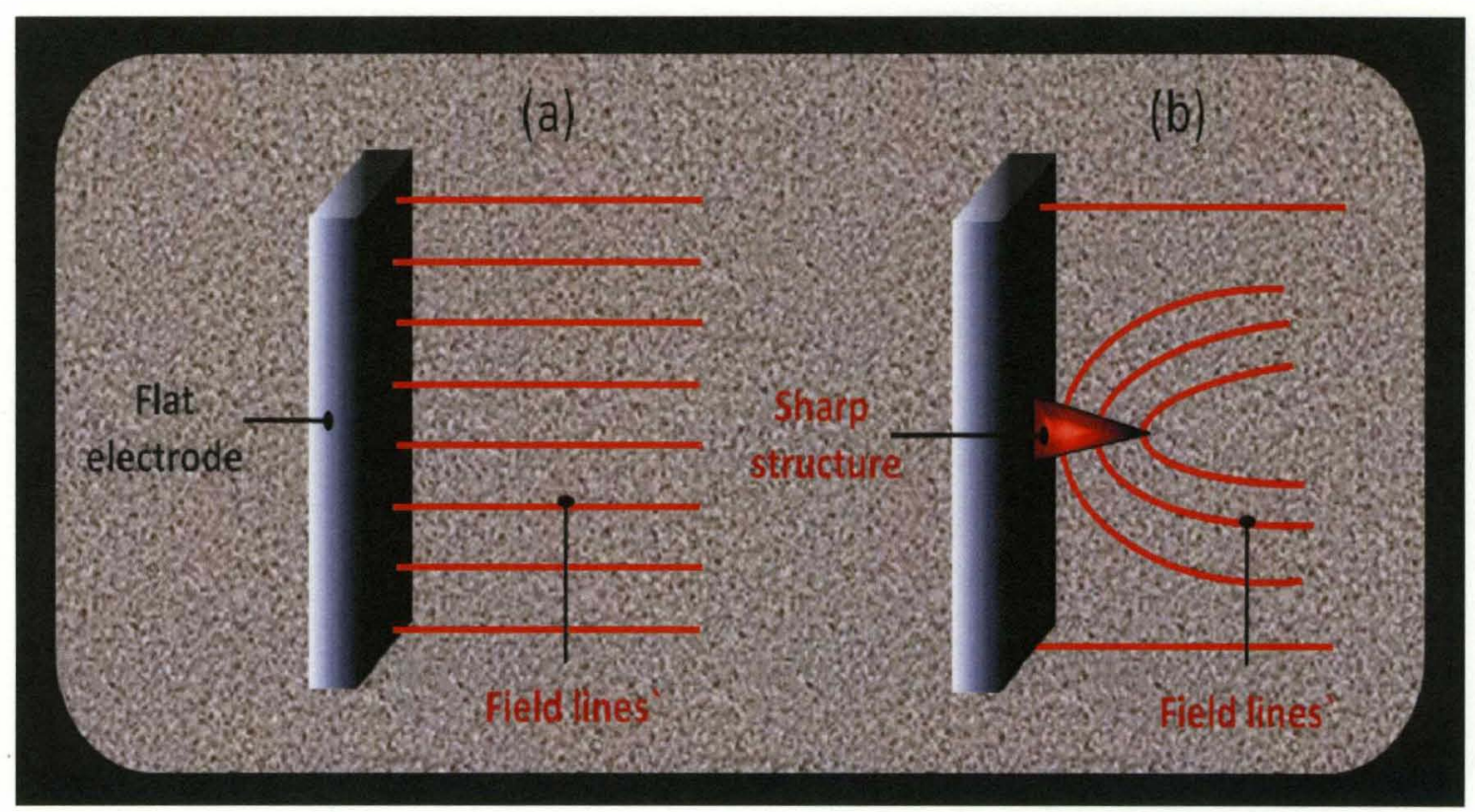

Figure 2-6: The electric field lines near (a) a flat surface and (b) a sharp structure. ${ }^{62}$ Although is it highly desirable to have high $\beta$, there is a trade off on the cathode's effective emitting area and value of $\beta$. Hence, design and fabrication of relatively high density cathodes that are efficient field emitters is needed. Nanostructures in particular are able to fit into this category of emitters that can easily combine high electrical 
properties, high amplification factor as well as high packing density as will be demonstrated in the following chapters.

Two configurations are generally employed to characterize and to fabricate a field emission system. Diode arrangement is the basic one where two electrodes are placed next to each other within vacuum. It can be modeled as an electrical circuit where the emission current is the current circulating within the circuit is due to the emitted electrons and is obeying F-N law instead of Ohm's law (Figure 2-7). Electrons extracted are accelerated towards the collector by the electric field present within emitter-collector gap. The other configuration is triode arrangement that has a significant impact on the performance of vacuum microelectronic field emission based devices as the ejected electrons get amplified towards the anode.

Triode configuration consists of three terminals: cathode (emitter), a grid which is a positively charged metal that can be placed as close as possible to the emitter, and the plate or anode (collector). The electric field created on the grid is able to repel/accelerate the extracted electrons and limit the number of electrons passing through. Figure 2-7 shows the circuitry of the field emission system set-up. It consists of two electrodes having opposite charges that are placed within the vacuum. The grid can be also integrated within the system to control the emitted electrons and enhance emission. 


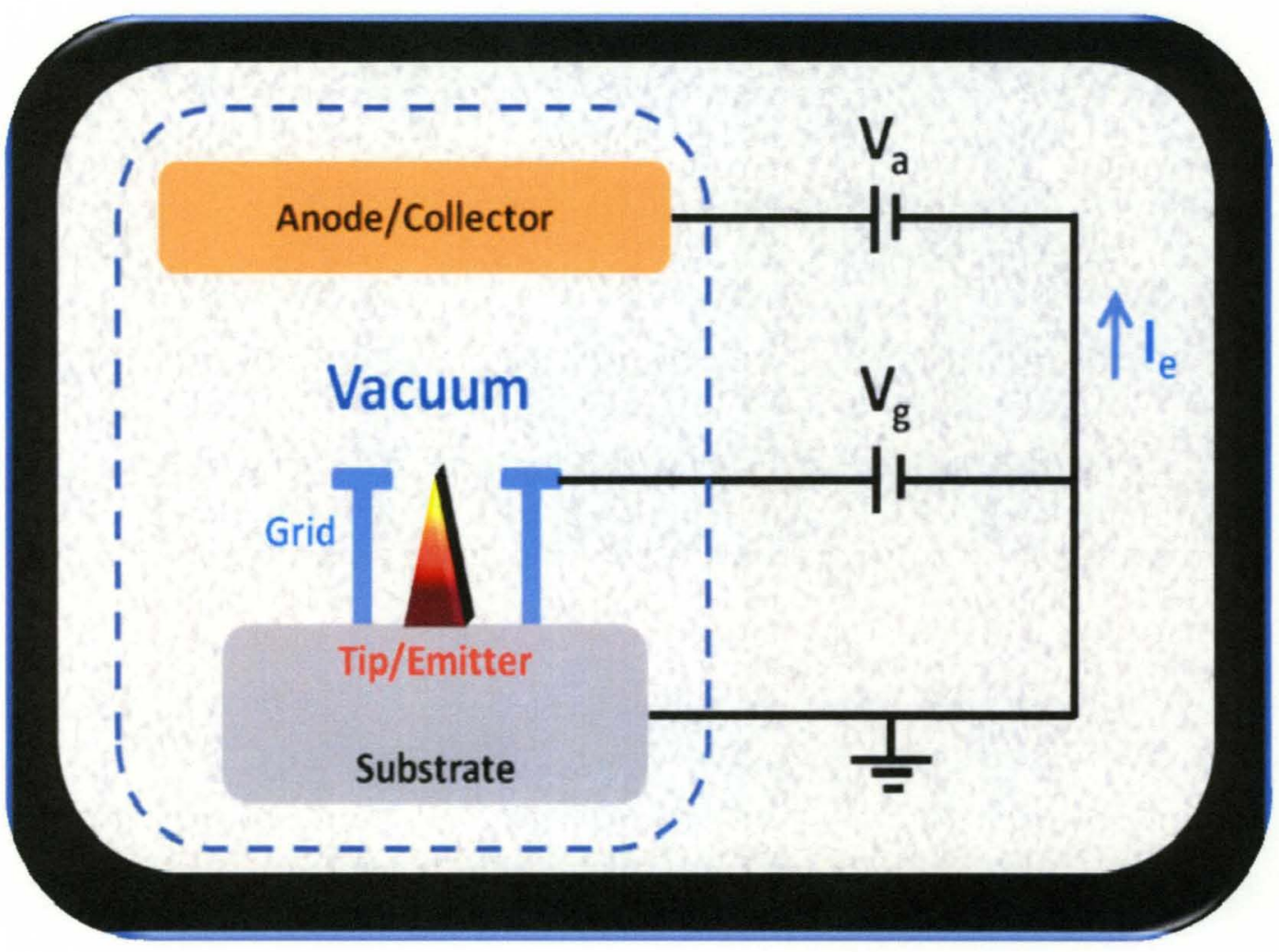

Figure 2-7: Triode configuration of a field emission characterization set-up. ${ }^{63}$ 


\section{2-3 Field Enhanced Thermionic Emission}

At high temperature and low field, the temperature dependence of the FermiDirac distribution is mainly responsible for variations in the emission current; hence, thermionic emission predominates EE. At high field strength and low temperature, field dependence of the barrier shape is principally responsible for variations in the emission current; consequently, field emission predominates the EE. Thermionic field emission takes place when the tunneling phenomena along with thermal excitation significantly contribute to the total emission current. Therefore, the electron can climb over the barrier, and penetrate through or some will climb while others will tunnel through simultaneously. Consequently the emission current increases with the temperature and the applied field. Field enhanced thermionic emission (FETE) takes place when the field strength is enough to band the potential barrier and reduce the work function without initiating tunneling.

While the theories of FE, TE and FETE of electrons from metals have been well studied, little work has been devoted to the intermediate region where temperature and field significantly contribute to the emission. The reason is the narrowness of the region itself and its sensitivity to little change in the pair temperature-field values. Whereas FETE has drawn lot of focus and attention due to the low power consumption compared to TE enabling wide applications such as energy conversion.

During TE process the emitted electrons have zero velocity and tend to form an

electron cloud, known as space charge, near the hot emitting surface, resulting in an extra potential barrier and limiting the number of electrons that can reach the collector. Therefore, an extra force in the form of electric field needs to be introduced to accelerate 
the thermally emitted electrons towards the collector. The larger the electric field, the larger the resulting current of electrons.

At thermal equilibrium, the potential barrier is equal to the material's work function minus the image charge potential when space charge effect is not present (Figure 2-5).

$$
V_{x_{0}}=E_{F}+\Phi-\frac{e^{2}}{4 x}
$$

Application of an electric field will farther reduce the barrier height and produce a triangular barrier type,

$$
V_{x}=E_{F}+\Phi-\frac{e^{2}}{4 x}-e E x
$$

The resulting reduction in the work function can be expressed as:

$$
\Delta \Phi=-e E x-\frac{e^{2}}{4 x}
$$

Image charges build up in the metal electrode as carriers approach the metalvacuum interface. The potential $(-e 2 / 4 x)$ associated with these charges reduces slightly the effective barrier height even in the absence of an applied field. Application of an external electric field has two benefits; reduction in the barrier height due to $(-e E x)$ term contribution and decrease in the barrier width. The ability to reduce these two quantities is very important in designing an efficient $\mathrm{EE}$ based device. 
The lowering of the EE barrier is commonly referred to as the Schottky effect. The amount by which the barrier lowers is proportional to the square root of the electric field via the equation:

$$
\Delta \Phi=\sqrt{\frac{e^{3} E}{4 \pi \varepsilon_{0}}}
$$

The Equation 2-20 shows that field strength of the order of $10^{3}$ Volts/ $\mu \mathrm{m}$, is easily achievable using nanotips and results in an approximately $1.2 \mathrm{eV}$ decrease in the work function. The higher the field strength the higher is the reduction in the barrier height.

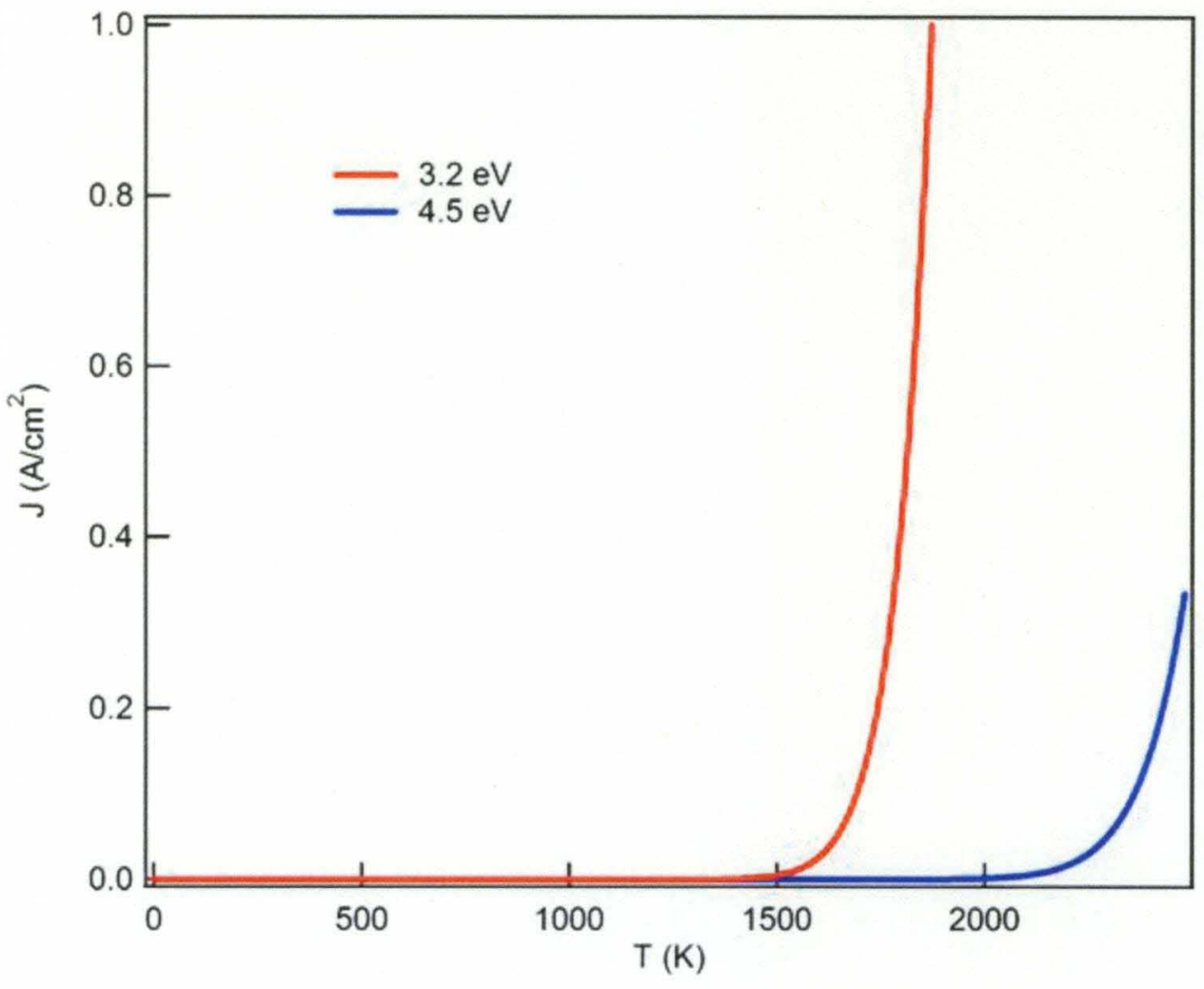

Figure 2-8: The emission current of a tungsten filament and treated tungsten as function of temperature. ${ }^{64}$ 
Once the work function is reduced, electrons can easily climb the potential barrier; hence; EE will occur at lower temperatures than in the case of pure TE. As will be discussed in the next chapter, a little decrease in this quantity will have a large increase in the emission current and decrease in the emission turn-on temperature. The resulting device will consume less power and have longer switching time. Figure 2-8 illustrates the emission of a tungsten filament $(4.5 \mathrm{eV}$, red curve) and treated tungsten (blue curve) as a function of temperature. A small percentage change in temperature (5\%) in the emitting region results in a big change in the emitted current (by about factor of 2). On the other hand, a decrease in the work function by $0.5 \mathrm{eV}$ increases the emission current by a factor of $(\exp [1 /(2 \mathrm{KT})] \sim 3500)$ at $1773{ }^{\circ} \mathrm{C}$. The smaller the work function the easier the electrons can escape and contribute to the emission current.

Figure 2-5 shows the potential barrier existing within the metal with and without the presence of an applied electric field. The work functions $\Phi$ and $\Phi_{\text {eff }}$ denote respectively the old and new barrier that electrons see before they can get to the vacuum level.-Hence, the emission formula should consider change in the work function:

$$
J=A^{\prime} T^{2} e^{-\left(\frac{\Phi_{e f f}}{K_{b} T}\right)}
$$

Using equation 2-4, the current density is expressed as:

$$
J=J_{0} e^{\frac{\Delta \Phi}{K_{b} T}}
$$

Where $J_{0}$ usually referrers to the zero field current density. 
Therefore, the main determining factors for the all types of EE are temperature, the electric field and the material's work function. The ability to design an efficient electron emitter based device can be manifested in obtaining EE at low temperatures and low electric fields. This is achievable by using low work function emitting surfaces that can withstand rough conditions such as high temperature and high vacuum environment. As will be demonstrated in the next chapter, just few materials are appropriate for TE while a larger selection of cathodes can be used for FE. Surface treatment of the cathode by thin film coating of alkaline earths materials is commonly used to lower the work function of the emitting surfaces. Another technique is to apply a relatively high electric field to lower the potential barrier height (the work function), which is easily achieved by taking advantage of local field enhancement when using nanostructures, Field enhanced thermionic emission is based on the field enhancement to lower the potential barrier and the ability to achieve high local field strengths at moderate low voltage. Thus, nanostructures can be an essential element of TE and FE based devices. 


\section{2-4 Material Selection and Emitter Design and Modulation}

Various parameters determine the choice of the cathode materials to be employed as thermionic emitters. These parameters include the compatibility of cathodes with the environments in which they are operated, the work function of the cathode which needs to be as low as possible; the emission temperature of operation at which appreciable emission takes place is desired to always be low, and finally, the melting temperature when the evaporation of the cathode starts to be significant has to be very high. In addition the maximum current density that can be extracted from the emitter and the emitters' lifetime also affects the choice of material and can limit its application. Therefore, the amounts of materials that can satisfy the aforementioned conditions are limited. An Extensive search for materials and treatment and processing of hot cathodes has been performed resulting in exhaustion during the time of exploring electron tubes. However, the result of this search was encouraging and new processes were discovered to lower the work function and increase the cathodes' efficiency, nevertheless, tradeoff between TE properties is always present.

\section{2-4-1. Thermionic emission materials}

Hot emitters are classified into two categories, directly and indirectly heated cathodes, depending on the way the heat is generated. A directly heated cathode consists of heating a metallic filament to a white incandescence to initiate electron evaporation process. The filament is usually made of tungsten, has hairpin-like (v-shape) filament made of tungsten wire. A DC current is passed through the W-filament to heat it up to a temperature of $\sim 2500 \mathrm{~K}$, at which it emits practical number of electrons into the 
surrounding vacuum. Tungsten makes up most of this category of cathodes. However, in theory, any filament with high melting temperature should be able to emit electron once it is heated, directly or indirectly, to a white incandescence; however, good hot cathodes needs to produce appreciable emission current to be of use for the applications.

The indirectly heated cathodes consist of placing the emitters on an electrically isolated but thermally conducting heater to increase the cathode's temperature to the desired onset and emission value. The indirectly heated cathodes are usually covered with a thin emissive layer which is typically the oxides. The oxides can react with some emitting materials to significantly reduce their work function. The indirect heating technique is adopted in the present work by reason of its advantages over the direct heating technique:

The indirect heating technique is adopted in the present work by reason of its advantages over the direct heating technique:

- With an indirect heating system, any shape and area of the cathode can be used, instead of just a filament. Moreover, higher current density can be extracted.

- The thermal run way can significantly be reduced due to the absence of joule heating.

- Indirect heating increases the cathodes' life time when compared the direct heating. (No high current has to pass through the emitter-substrate system)

Below are few examples of materials that are commonly used in many applications as hot cathodes. The choice of materials is governed by the need of high melting temperature of the emitter, low work function or combination of both. The first material is tungsten: 


\section{a. Tungsten}

Polycrystalline tungsten was one of the materials that attracted much attention

when the focus on TE started. Tungsten can function at high operating temperature (2500 $\mathrm{K})$, by which an emission density up to $400 \mathrm{~mA} / \mathrm{cm}^{2}$ can be produced from pure polycrystalline tungsten filament. Tungsten has a lower work function of about $4.6 \mathrm{eV}$ versus other metals; however, it requires the filament to be heated to high temperatures to initiate appreciable emission current. Since the power drawn by any hot object is proportional to the fourth power of its temperature, large amount of heat power is wasted when TE takes place which lowers the efficiency of tungsten filament. Even with the lowest efficiency and high work function with respect to other materials, tungsten is still used in applications requiring high power due to high melting point of $3650 \mathrm{~K}$, greater mechanical strength, its high resistivity to ion bombardments and longer life. In addition, tungsten filaments are easy to manufacture, have high life time and does not require an expensive fabrication process.

\section{b. Thoriated Tungsten}

Thorium (Th) is one of the materials that once added to tungsten, decreases its work function and increases its emissivity. Adding a small quantity of thorium to tungsten reduces significantly its work function to about $2.6 \mathrm{eV}$, which is $43 \%$ lower than pure tungsten resulting in a lower onset TE temperature of $1700 \mathrm{~K}$ and an operating temperature of $2173 \mathrm{~K}$.

Thoriated tungsten, once used in a thermionic diode configuration, is proven to consume less power at higher efficiency when compared to pure tungsten. However, it is not as robust as tungsten. 


\section{c. Oxide coated cathodes}

Oxide coated cathodes consist of depositing thin film of a mixture of metallic oxides onto a ribbon of nickel or nickel alloy. The most frequently used oxides are $\mathrm{BaO}, \mathrm{SaO}$, and $\mathrm{CaO}$ produced from the alkaline earths metals such as barium, strontium, and calcium. The emissive coatings are either a double which is a mixture of $\mathrm{BaO}, \mathrm{SrO}$ oxides or triple that is a mixture of $\mathrm{BaO}, \mathrm{SrO}$ and $\mathrm{CaO}$. Usually the oxide coating is applied to the nickel ribbon in the form of the corresponding carbonates (i.e. $\mathrm{BaCO}_{3}$ ) in order to stabilize the cathode during emission. During the coating process, activation is carried out by heating the cathode to decompose the carbonates. At room temperature and higher, electrons are excited to the conduction band of the coating by donors which are distributed all over the thin film. At the cathode operating temperature, the conduction electrons gain enough energy to overcome the work function of the oxide coating and escape into the vacuum producing the TE current. The principal donor in the coating is due to an excess of metallic atoms of the alkaline earths in the oxide, which is produced during the cathode activation step. ${ }^{65}$

The oxide coating can lower the cathode's work function down to $1.1 \mathrm{eV}$. They operate at comparatively low temperatures; typically, they operate at 1073-1273 K and they can achieve even smaller $\mathrm{T} \sim 750 \mathrm{~K}$. In addition, they have higher thermal emissivity than pure tungsten. However, they can't withstand high voltages; therefore, it is used only in low power applications. Moreover, the inconvenience of using the oxide is that they tend to blister, get destroyed by ion bombardment, and easily get poisoned in a rough operating environments. ${ }^{66}$ The activated electrodes can be destroyed by contact with chemicals such as oxygen, carbon dioxide, water, aluminum, or silicates. ${ }^{66}$ 


\section{d. Dispenser cathode}

Dispenser cathodes are based on a porous tungsten matrix that is impregnated with a Ba-based multi-component oxide. ${ }^{67}$ Surface layers are often sputter deposited onto the impregnated and machined surface of the dispenser body to tailor the emission properties for specific temperature ranges, environments and applications. The composition is essentially a ternary-oxide that contains $\mathrm{BaO}, \mathrm{CaO}$ and $\mathrm{Al}_{2} \mathrm{O}_{3}$ in varying proportions. Common compositions are 3:1:1 and 5:3:2 $\mathrm{BaO}: \mathrm{CaO}: \mathrm{Al}_{2} \mathrm{O}_{3}{ }^{68}$

\section{e. Boride cathodes}

Hexaborides were very successfully employed as hot cathodes, for high brightness applications. Some of them produce high current while resistant to poisoning from ion bombardments and harsh vacuum environments. The most commonly used borides are lanthanum hexaboride $\left(\mathrm{LaB}_{6}\right)$ and cerium hexaboride $\left(\mathrm{CeB}_{6}\right)$, which is also another type of coatings used by hot cathodes to resists against poisoning and for high-

current applications ${ }^{54}$ Boride cathodes are also characterized by lower work function (i.e. $2.5 \mathrm{eV}$ ), have longer lifetime and are much brighter than the tungsten. When operated at $1700 \mathrm{~K}$, the lifetime can reach up to 500 hours, and the brightness around $3.10^{6}$ $\mathrm{A} / \mathrm{cm}^{2} / \mathrm{Sterad}$. The fabrication process of $\mathrm{LaB}_{6}$ cathodes is more expensive than tungsten hairpin and they requires higher vacuum to operate. ${ }^{69}$

\section{f. Novel cathodes}

In addition to the listed oxides and borides, other materials are starting to emerge to be used within VME and VNE technology. New materials have been developed and proven to be effective hot cathodes; such as CBNs, diamond and carbon nanofibers. ${ }^{70}$ 
The need for materials that combine the resistance against harsh conditions and low work function makes it essential to create a new generation of hot cathodes that optimize the operating conditions and open a new set of applications. The oxide coatings of CNTs have been reported with a $2.1 \mathrm{eV}$ for the resulting material's work function. ${ }^{71}$ 
Table 2-2: Selection of TE materials

\begin{tabular}{|c|c|c|c|c|c|}
\hline \multicolumn{2}{|c|}{ Cathode's materials } & $\begin{array}{c}\Phi \\
(\mathrm{eV})\end{array}$ & $\underset{\left(\mathrm{A} / \mathrm{cm}^{2}\right)}{\mathbf{J}}$ & $\begin{array}{c}\mathbf{T} \\
\left({ }^{\circ} \mathbf{C}\right)\end{array}$ & Ref. \\
\hline \multirow[t]{4}{*}{ Metals \& Alloys } & Tungsten (W) & 4.6 & 2.52 & 1500 & 72 \\
\hline & Molybdenum (Mo) & $4.36-4.95$ & 8 & 1400 & 73 \\
\hline & Barium tungsten bronze & 2.6 & $6.6 * 10^{6}$ & 570 & 74 \\
\hline & Cesium tungsten bronze $\left(\mathrm{Cs}_{\mathrm{x}} \mathrm{WO}_{3}\right)$ & 3 & $0.05 * 10^{-6}$ & 721 & 75 \\
\hline Borides & Lanthanum hexaboride $\left(\mathrm{LaB}_{6}\right)$ & 3 & 30 & 1625 & 76,77 \\
\hline $\begin{array}{c}\text { Oxides } \\
\text { (coating) }\end{array}$ & Barium strontium oxide & 2.6 & 1.6 & 925 & 78 \\
\hline \multirow[t]{2}{*}{$\begin{array}{l}\text { Carbon-like } \\
\text { cathodes }\end{array}$} & Nanocrystalline diamond & $1-3$ & & $800-1050$ & 79 \\
\hline & Carbon nanotubes & 4.8 & $15 * 10^{-3}$ & 1437 & 80 \\
\hline $\begin{array}{l}\text { Li-containing } \\
\text { materials }\end{array}$ & $\beta$-eucryptite & & $1.9 * 10^{-6}$ & 777 & 81 \\
\hline
\end{tabular}

Table 2-3: Effect of work function on the TE properties.

\begin{tabular}{|c|c|c|}
\hline Material & $\Phi(\mathbf{e V})$ & $\mathbf{T}(\mathbf{K})^{(*)}$ \\
\hline Tungsten $(\mathbf{W})$ & 4.6 & 2608 \\
\hline Thoriated Tungsten $(\mathrm{Th}-\mathbf{W})$ & 3.2 & 1870 \\
\hline Oxide & 1.5 & 942 \\
\hline
\end{tabular}

${ }^{(*)}$ Emission temperature needed to produce $1 \mathrm{~A} / \mathrm{cm}^{2}$. 


\section{2-4-2. Electron emission validity regions}

Using the Fermi-Dirac distribution for a free electron gas in the metal and the classical image force barrier at the metal-vacuum interface, Murphy and Good were able to develop a model for the regions of temperature and electric field in which the electron emission is possible. In addition, they used the general equation that governs the electron emission from a metal to deduce the generalized equations that govern each type of electron emission. ${ }^{46}$

The total emission current density is obtained by integrating over all energies, the product of the penetration probability and the number of incident electrons per second per unit area with respect to the total electron energy $W$. The emission current is a function of the field, temperature and work function; it is expressed as: ${ }^{57}$

$$
\begin{aligned}
& J(E, T, \Phi)=e \int_{0}^{\infty} D(E, W) N(T, \Phi, W) d W \\
& J(E, T, \Phi)=\frac{k T}{2 \pi^{2}} \int_{-w_{a}}^{\infty} D(E, W) \ln \left(1+e^{\frac{-(W-\zeta)}{k T}}\right) d W
\end{aligned}
$$

Where $J$ is the emission current density, $E$ the electric field, $T$ the temperature, $D$ the penetration probability, $N$ the number of electrons, $W$ is the energy and $W_{a}$ is the effective constant potential inside the metal $k$ is Boltzmann constant, $(-\zeta)$ is the work $\Phi$. The transmission coefficient is obtained via the solution of the one dimensional time independent Schrödinger equation. 
Murphy and Good had established a set of conditions, a range of temperature and field for the three types of emission (TE, FE and TFE) along the corresponding expresion for the emission current desnity by using an approximation technique to resolve the integral of Equation 3-3. ${ }^{46}$ The approximation is valid only for values for the work function, field and temperature. The computed conditions while solving for the existence of solution of Equation 3-3, define the validity regions of each type of emission. The model is also able to reproduce the Richardson-Schottky, and Fowler-Nordheim equations.

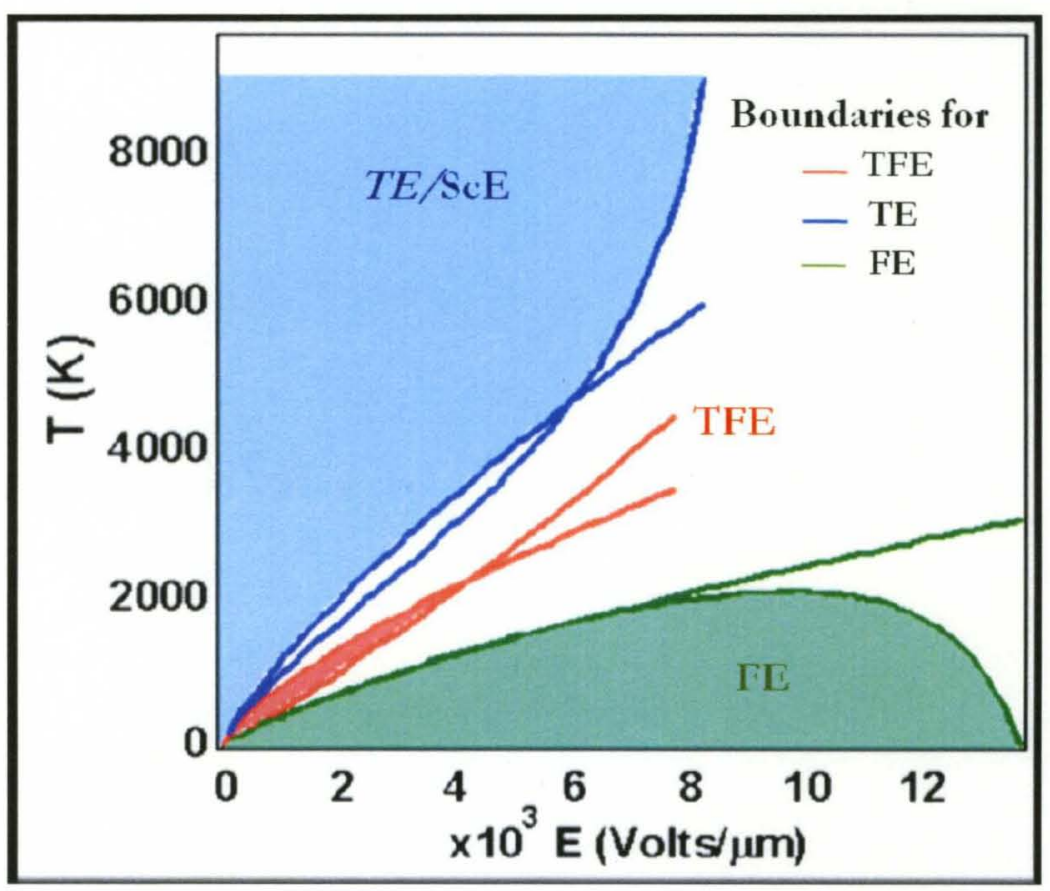

Figure 2-9: The validity regions of the three types of electron emission (TE, FE and TFE) for a $5 \mathrm{eV}$ work function cathode. ${ }^{82}$

In Figure 2-9, three regions can be identified according to the values of temperature and electric field. At high temperature and zero fields, the temperature dependence of the Fermi-Dirac distribution is behind the variations in the emission current; hence, TE predominates. Field Enhanced Thermionic Emission (FETE) takes 
place when the field strength is enough to band the potential barrier and reduce the effective work function without initiating tunneling process. Hence, FETE dominates electron emission. The TE and FETE is valid only within the blue shaded region of Figure 3-9.

At high field strength and low temperature, field dependence of the barrier shape is principally the origin for the variations in the emission current; consequently, FE dominates the electron emission and it is valid only within the green shaded region.

An intermediate region appears at moderate values of temperature and field. This region defines the Thermionic Field Emission (TFE) mode. TFE takes place when the tunneling phenomena along with the thermal excitation, significantly contribute to the total emission current. Consequently, the emission current increases with the temperature and applied field. The boundaries of the temperature and field, within which TFE takes place, are intermediate between TE and FE region. The boundaries are defined by the red curves of Figure 2-9. The TFE occurs at the narrow red shaded region which is sensitive to the changes in the parameters and practically difficult to realize.

Murphy-Good theory and boundary conditions are used to model the validity regions for the three types of emission for different materials.

In the case of FE, since the barrier width of $1 \mathrm{~nm}$ or less is enough to initiate cold emission, the validity region area shrinks down as the work function decreases. Therefore, the tunneling distance gets smaller and the easiest is the cold emission to occur. 


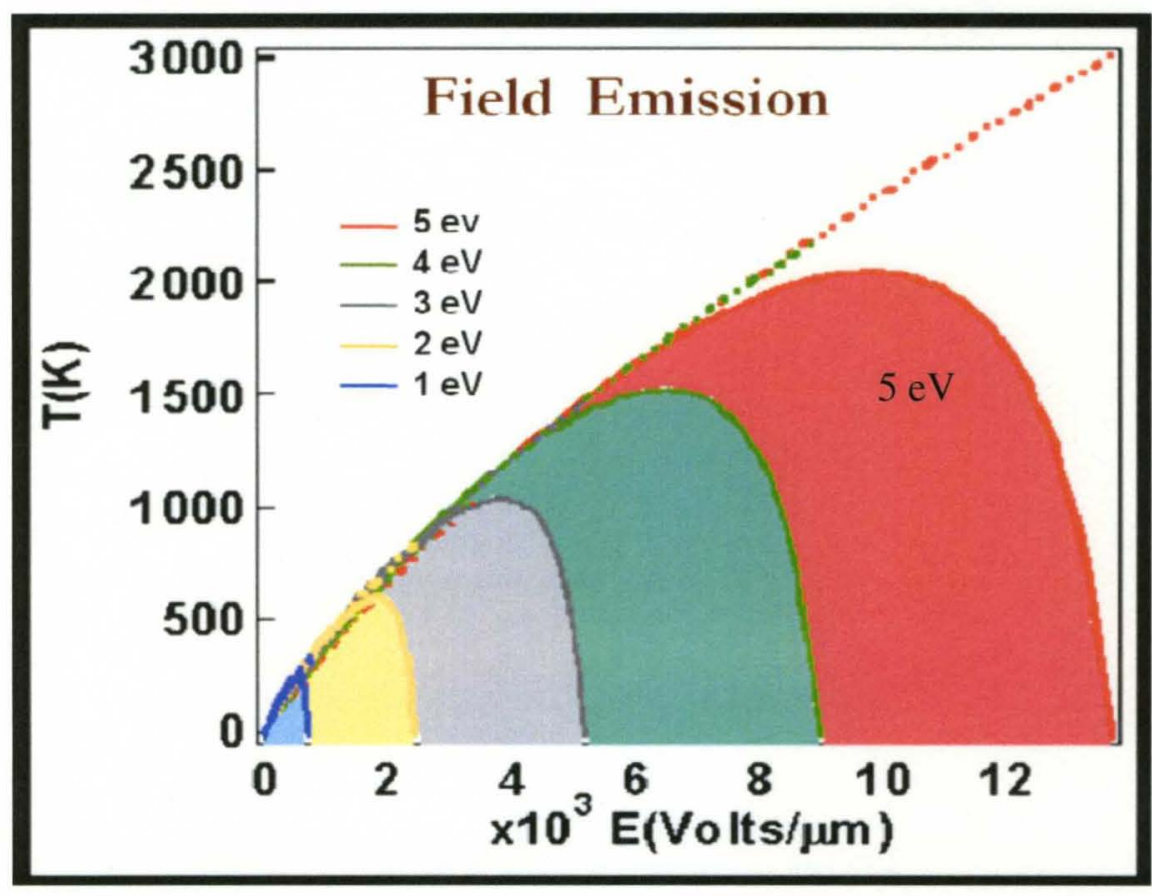

Figure 2-10: (a) FE boundaries for five different materials

(b) TE ad ScE boundaries for five different materials. ${ }^{83}$

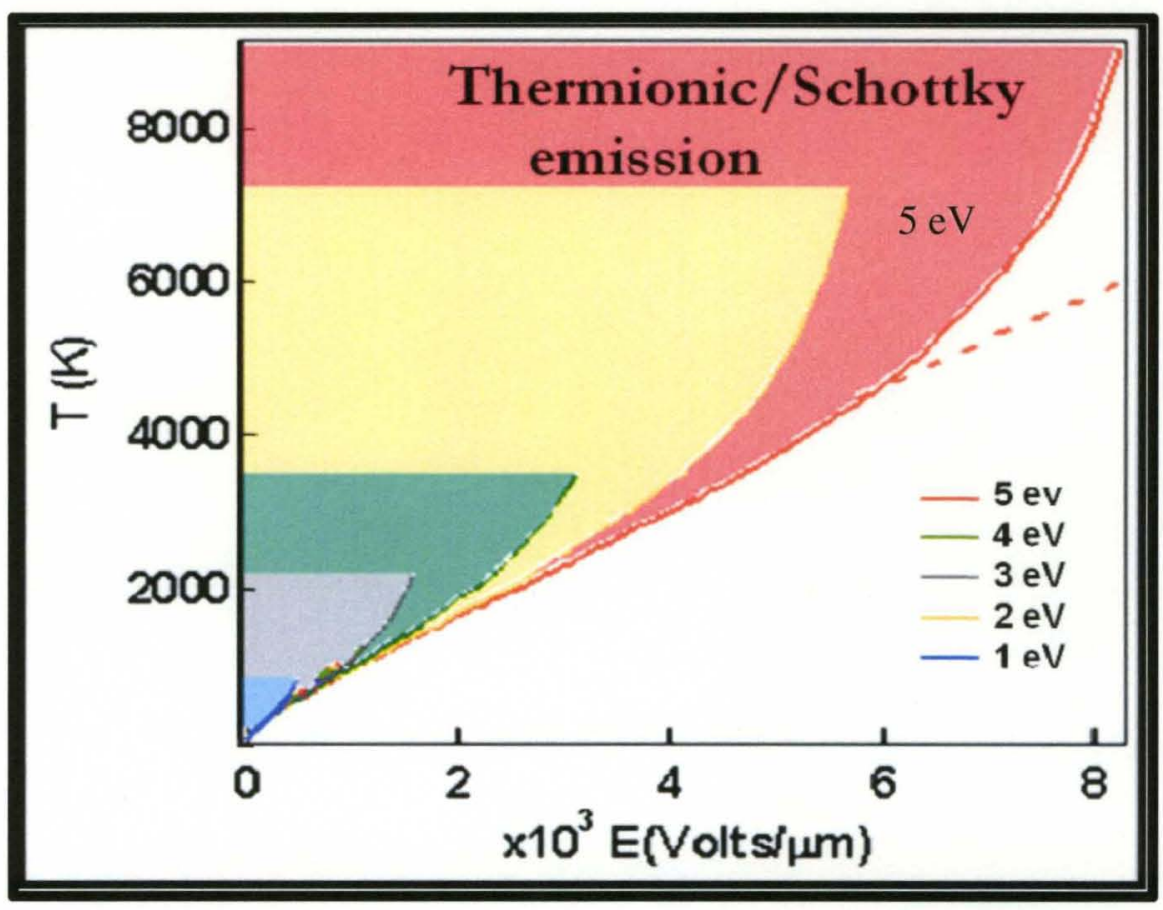

Figure 2-11: TE ad ScE boundaries for five different materials. ${ }^{84}$ 
Figure 2-10(a) displays the boundaries of the FE and the shrinking of the validity region as the work function decreases. Low work functions materials require less field strength than those of high work function. According to these models, for FE to take place; field strength of $13 * 10^{3}$ Volts $/ \mu \mathrm{m}$ could be required for a $5 \mathrm{eV}$ material, which is more than $90 \%$ than what is required to stimulate FE from a $1 \mathrm{eV}$ cathode.

Figure 2-10(b) displays the boundaries of the TE and ScE. TE takes place at zerofields, while ScE is present at the introduction of an electric field. In this case, the validity region of emission opens up with the increase of both temperature and field. The validity region is limited by the materials melting temperature and by the minimum value of temperature at which the cathode produces a detectable emission current. The lower the work function is, the narrower the validity region and the less energy is spent to extract electrons. To explain this by numbers and according to the model and current estimations, the operating temperature of a $5 \mathrm{eV}$ cathode is $85 \%$ higher than a cathode having a work function of $1 \mathrm{eV}$. 


\section{2-4-3. Field screening effect}

The behavior of a conductor in the presence of an electric field changes as soon as another conductor is placed at its proximity. This is also the case when an electric field is applied between an array of nanoemitters and an anode. Each emitter screens the field, especially when the inter-emitter distance is very small with respect to the emitter's height. The electrostatic field screening between the emitters is the decrease of the expected field amplification at the tip when a field is applied at its base. In fact, the field amplification factor drops as the inter-emitter spacing attains a distance less or equal to the emitter's height. This effect affects the field-emission properties as well as the performance of a FE device. ${ }^{14}$

An electrostatic simulation of an electric field around the emitters that are $5 \mu \mathrm{m}$ in length and sub $100 \mathrm{~nm}$ in diameter is performed. The inter-emitter distances are taken to be smaller, equal and longer than the emitter's length. Figure 2-11 illustrates the simulation results and showing the equipotential lines and the electrostatic field distribution for conducting emitters with varying inter-emitter distance. For an individual emitter, the electric field is very intense at its tip. The field enhancement is largest for well spaced emitters and decreases when the inter-emitter separation becomes comparable to the double of the emitter height. ${ }^{14}$

Based on the simulation, the following statements can be concluded:

- The super sharp tips such as nanostructures should amplify the field better than any preexisting cathodes (i.e. microfabricated emitters).

- A FE based device with highly dense emitters will operate at the lowest emission efficiency as the threshold field is high and current density is low. 
- $\quad$ Close-packed arrays of emitters having high aspect ratio are not ideal for FE applications. However, loose-packed arrays lower the emission current density. Therefore, there is a tradeoff between the aspect ratio and the emitters packing density. To produce highly efficient FE devices and optimize the emission current density one must design an emitter array where the field screening effect is effectively minimized by moderate density and aspect ratio.

The experimental data demonstrates that field emission from nanoclusters, which have small aspect ratio and are closely packed, is considerably appreciable. ${ }^{85}$

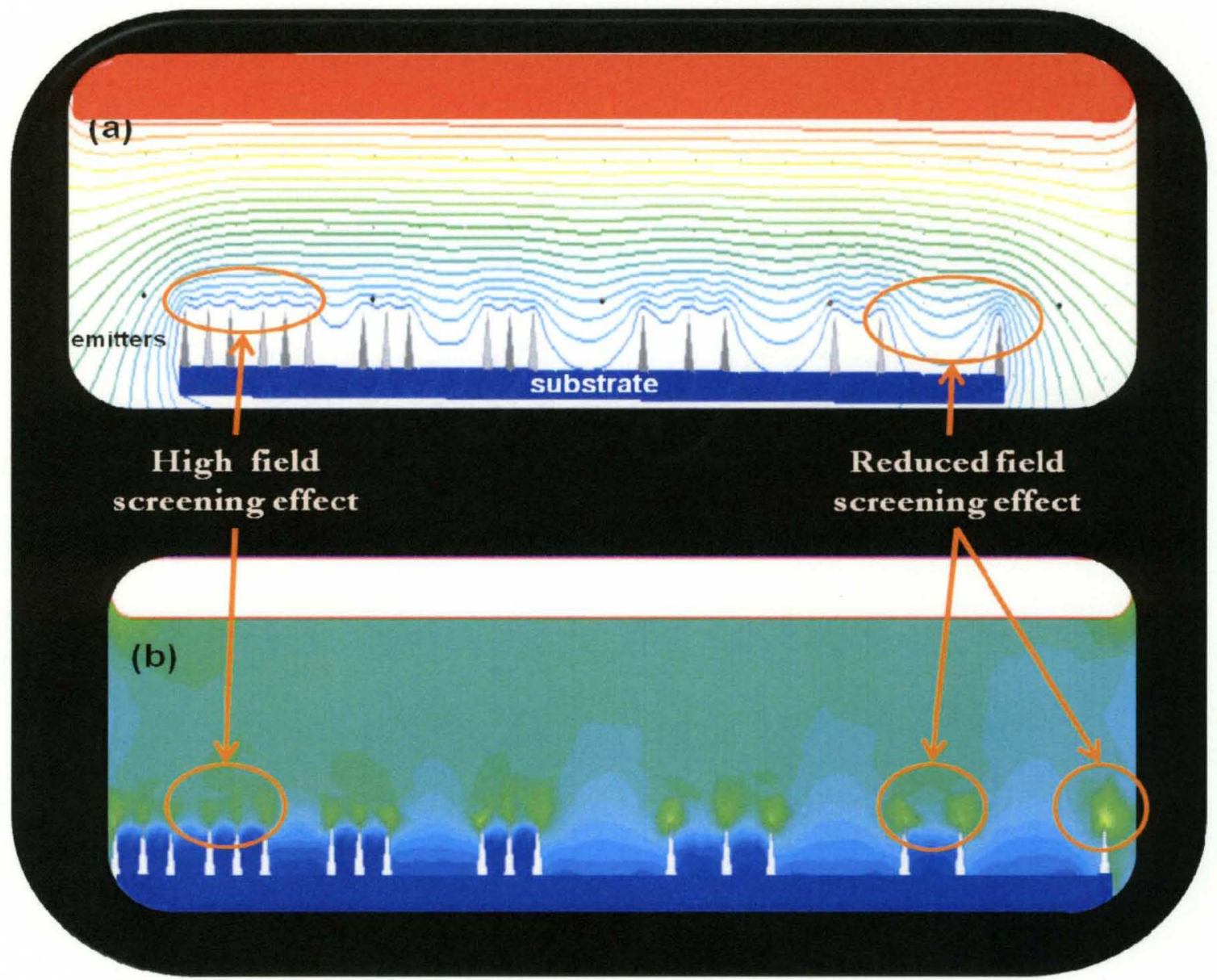

Figure 2- 12: Simulation of (a) the equipotential lines and (b) the electrostatic field distribution for conducting nanoemitters having different inter-emitter separations. ${ }^{86}$ 


\section{CHAPTER III}

\section{EXPERIMENTS}

This chapter reports on the measurements used for FE, TE and FETE from several nanostructured materials. Some of these materials are metallic or have metallic behavior, such as nanowires and metallic single wall carbon nanotubes while others are semiconducting structures, such as single and multiwall carbon nanotubes. The third category is insulators such as tungsten oxides nanowires. In this chapter, the techniques employed to synthesize or fabricate the aforementioned nanostructures, the experimental set-ups and tools used for EE characterization to optimize or discover new electron sources are presented. First, a brief discussion on materials synthesis is provided with emphasis on the novel nanostructures, CCNTs and M_SWCNT, growth processes. Then, the means by which thermionic emission and field emission from these nanostructures are investigated for bulk and In-situ measurements. Last, a description of the experimental set-up and its optimization for efficient electrons sources is included.

\section{3-1. Fabrication and synthesis of nanoemitters}

\section{3-1-1. Production of Multiwall carbon nanotubes}

Different processes generally used to grow MWCNTs include arc discharge, laser ablation and Chemical vapor deposition (CVD). ${ }^{87}$ Both arc discharge and laser ablation 
employ carbon precursor to provide the carbon sources required for the growth which is carried on at high temperatures ( $>1273 \mathrm{~K})$. CVD employs hydrocarbon gases as sources for carbon and metal catalyst particles as "seeds" of the growth. The temperature of the growth is relatively lower than the other two processes (773-1473 K).

CVD is regularly used today to grow many nanostructures. In particular, CVD is used extensively to grow CNTs directly on large area substrates. ${ }^{88}$ CVD systems can produce vertically aligned CNTs which are especially desirable for FE and FETE measurements. The first step is to prepare a thin film of nickel, iron or other metallic particles on a substrate. ${ }^{89}$ The size of the particles determines the diameter of the nanotubes. Then the substrate is heated in the furnace at around $1273 \mathrm{~K}$. Subsequently, to initiate the growth process two gases are usually driven into the furnace. One of the gases is the carbon source during the chemical reaction. The gasses diffuse and get adsorbed onto the substrate's surface where the chemical reaction takes place at the right temperature by the aid of a catalyst.

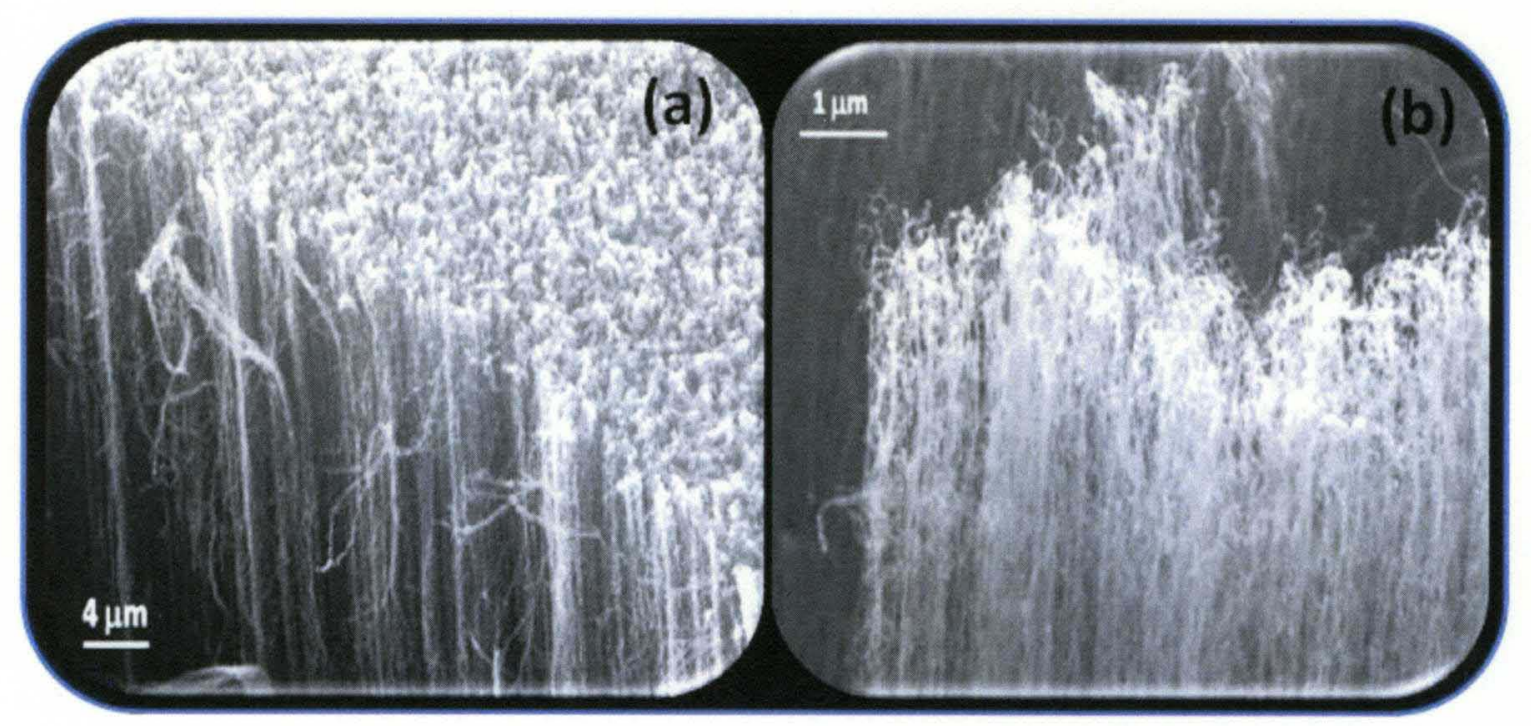

Figure 3-1 SEM images of MWCNTs vertically grown by CVD. ${ }^{90}$ 


\section{3-1-2. Synthesis of conical carbon nanotubes}

CCNTs are novel carbon nanostructures that are in the form of helical sheets wrapped into a cone making a shape of whiskers. These structures are introduced in the present work for EE characterization. The center of the CCNT consists of a hollow tube of constant diameter throughout the length of the structure. The cone tapers into a tip of diameter ranging from 10 to $30 \mathrm{~nm}$ and length of 2-20 um (Figure 3-2).

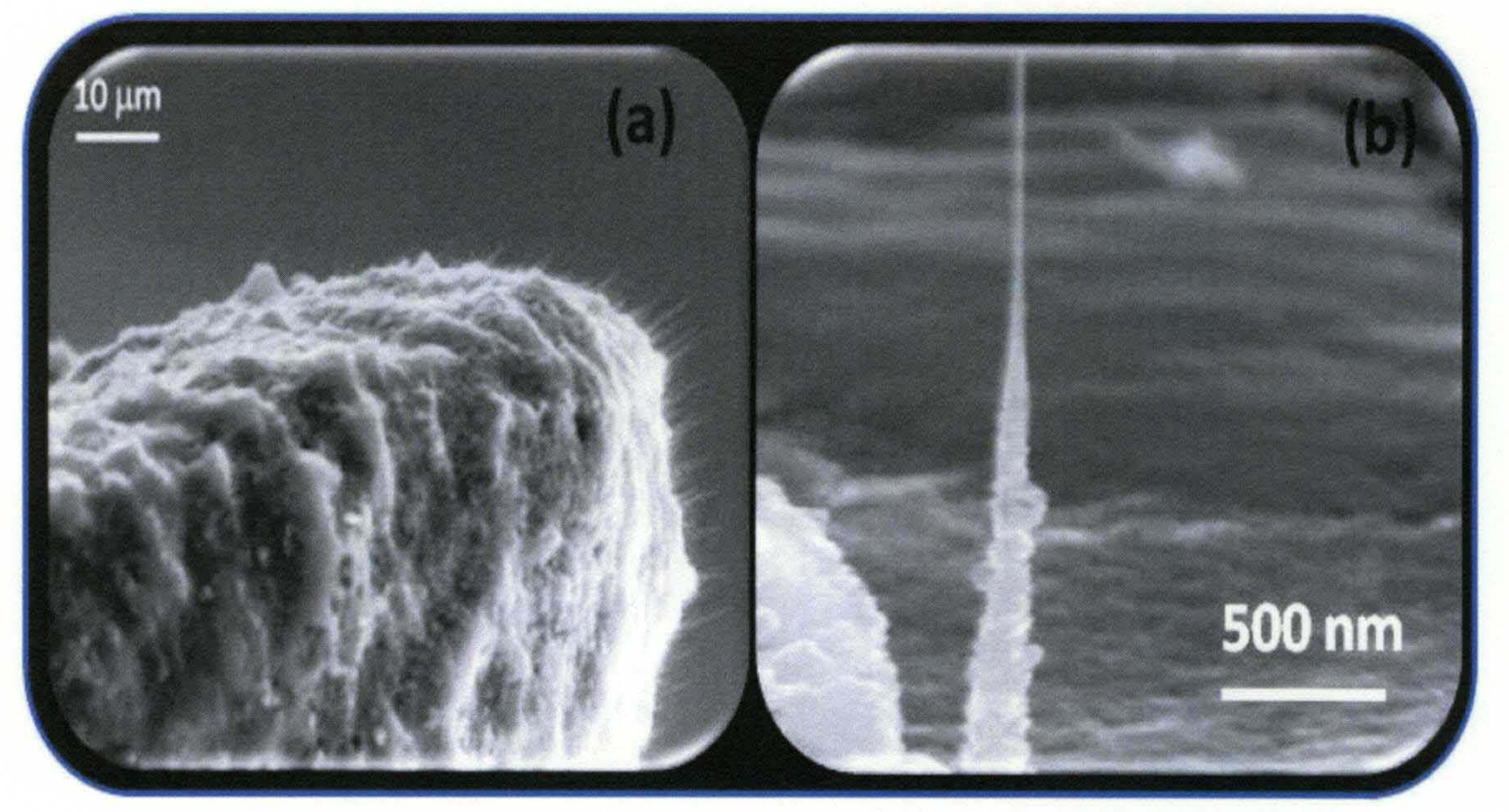

Figure 3-2 SEM image of CCNTS grown (a) at moderate density and (b) at low density together with carbon flakes at their facets. ${ }^{9}$

Mani et al presented the detailed method of synthesis of CCNTS. ${ }^{91}$ The process is somewhat similar to the CVD method used to grow carbon nanotubes. ${ }^{88}$ Usually, Platinum wire of diameter of $0.5 \mathrm{~mm}$ and $30 \mathrm{~mm}$ long are used as substrates. They are vertically placed on a graphite boat. The boat is immersed vertically into microwave plasma in a CVD reactor. The gas-phase composition is $1-2 \%$ of $\mathrm{CH}_{4} / \mathrm{H}_{2}$, which is atypical of carbon nanotubes growth. At the end of the deposition run, some regions of 
the substrate were found to be coated with a microcrystalline diamond film. These regions contain carbon whiskers that are $10-700 \mathrm{~nm}$ in diameter and up to $12 \mu \mathrm{m}$ in length are grown.

The whiskers have a pointed tip in the shape of pipette having base with submicron in size (Figure 3-2). There also was some minor faceting on the surface of the CCNTs. A Transmission Electron Microscopy (TEM) shows that these whiskers have a well-defined uniform hollow core, approximately 1-3 $\mathrm{nm}$ in diameter, extending throughout its length. The carbon bonds making up the CCNTs are $\mathrm{SP}^{2}$. In the process of growth, amorphous carbon film is deposited on a clean Pt wire and the CCNTs protrude above the film. Two steps are added to the growth process in order to produce a variety of structures including the change in the gas phase composition to vary the structural characteristics of CCNTs. Step 1 consists of carbon deposition using 1.35 vol \% methane in $200 \mathrm{sccm}$ of hydrogen followed by a deposition and etching with 1 vol \% methane in step 2. 


\section{3-1-3. Production of metallic single wall carbon nanotubes}

\section{a. Synthesis of M_SWCNTs}

SWCNTs synthesis involves the CVD method adopted in the CNTs growth. The CVD method results in high yield nanotubes having the metallic conductivity property.

The M_SWCNTs are grown from Fe nanocatalysts deposited on $\mathrm{Si} / \mathrm{SiO}_{2}$ substrate. The silica layer is $300 \mathrm{~nm}$ thick and thermally grown on a highly doped p-type Si wafer using dry oxidation method. The substrate is then immersed into a solution made out from 0.05 $\mathrm{mg}\left[\mathrm{Fe}\left(\mathrm{NO}_{3}\right)_{3} 9 \mathrm{H}_{2} \mathrm{O}\right]$ that is dissolved in a $1 \mathrm{ml}$ isopropanol solution for 1 minute. The substrate is then dipped into hexane for 10 seconds and air dried.

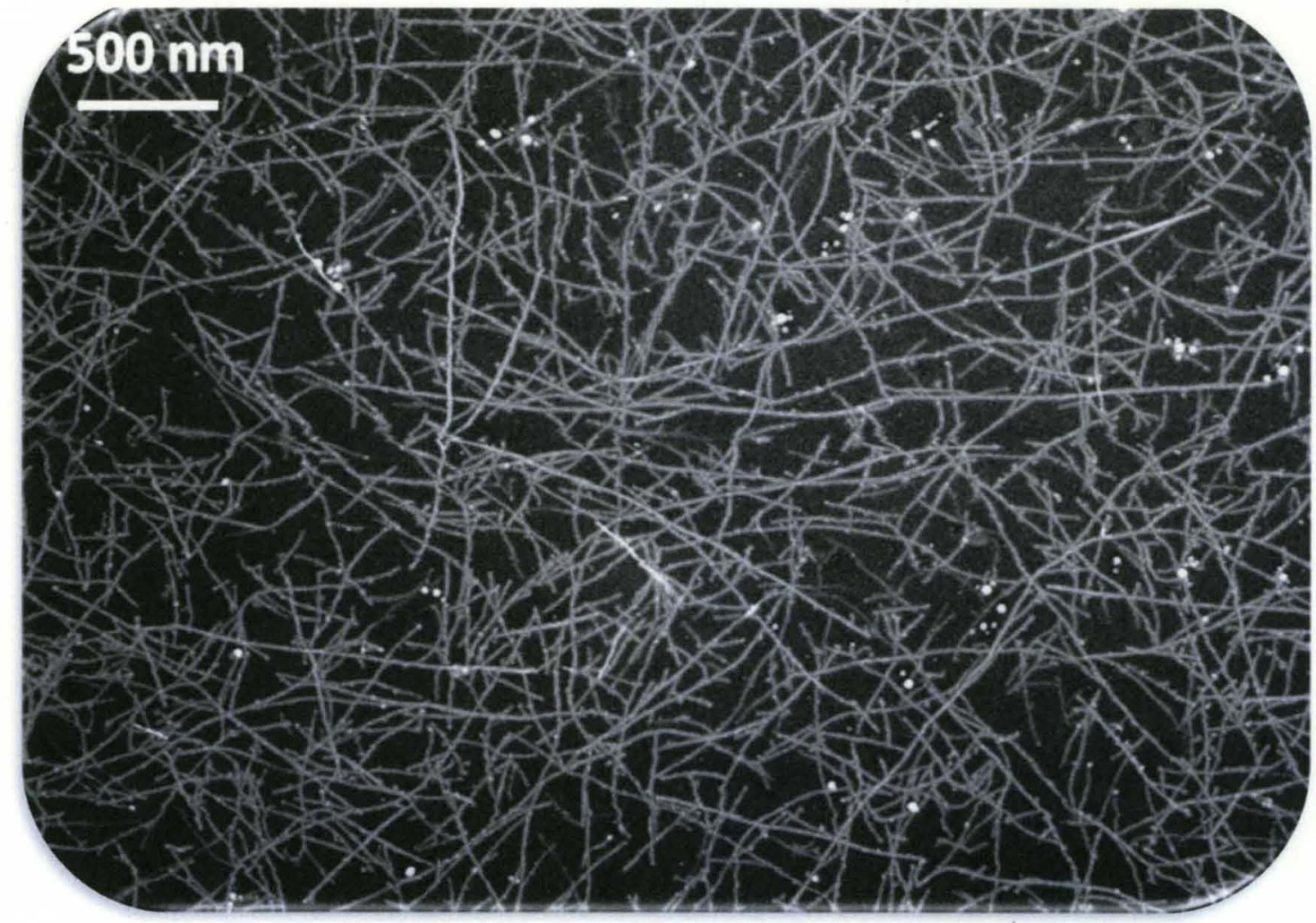

Figure 3-3: SEM image of M_SWCNTs grown by CVD system. ${ }^{92}$ 
Amorphous $\mathrm{Fe}_{2} \mathrm{O}_{3}$ particles get attached to the $\mathrm{SiO}_{2}$ surface then crystallize at 450 ${ }^{0} C$ under high purity He gas flow. Before M_SWCNT growth, the catalyst particles are reduced at $450{ }^{\circ} \mathrm{C}$ using (8:2) mixture of $\mathrm{H}_{2}$ and He gases at a flow rate of $700 \mathrm{~cm}^{3} / \mathrm{min}$. The temperature is then increased to $860{ }^{\circ} \mathrm{C}$ while gas mixture is flowing through the reactor. Methane $\left(\mathrm{CH}_{4}\right)$, the carbon source during $\mathrm{CVD}$ growth, is introduced into the reactor for $10 \mathrm{~min}$ at a flow rate of $300 \mathrm{~cm}^{3} / \mathrm{min}$, terminating the other gases streams. The furnace has been cooled down under $\mathrm{He}$ and $\mathrm{H}_{2}$ mixture. Figure 3-3 shows a SEM image of as grown M_SWNTs.

\section{b. Estimation of Semiconducting/Metallic ratio of SWCNT}

For a reasonable estimation of the ratio of metallic to semiconducting tubes, the integral intensities of the Raman radial breathing modes (RBMs) are used, which is defined as:

$$
\mathrm{R}=\mathrm{I}_{\mathrm{met}} / \mathrm{I}_{\text {sem }}
$$

Where, $I_{m e t}$ and $I_{\text {sem }}$ are the intensities of the metallic and semiconducting tubes respectively.

Each spectrum is the average of 50 individual Raman spectra and measured from different spots of the nanotube sample. The laser beam has about $1 \mu \mathrm{m}$ of diameter at the sample and the inter-spots distance of $10 \mu \mathrm{m}$. Two distinguishable regions in the RBM spectrum are observed, one in the $120-160 \mathrm{~cm}^{-1}$ range, which is assigned to semiconducting tubes $\left(S_{22}\right)$, and another band in the $160-230 \mathrm{~cm}^{-1}$ range assigned to metallic tubes $\left(\mathrm{M}_{11}\right)$ shows the Raman RBM spectra of the SWCNTs grown on the Fe catalyst using described procedure shows that for the samples RBM band of the as-grown 
tubes is dominated by metallic tubes $R=20.2$. The $G$-band spectra (Figure 3-4) displays a transitions from Lorenzian to Breit-Wigner-Fano line shapes for the corresponding sequence of the samples (Figure 3-4(b)). To obtain a reasonable quantitative estimation of the percentage of metallic tubes, a comparison of the integrated RBM peaks of the Raman spectra with the spectra of a reference sample is performed. ${ }^{93-95}$

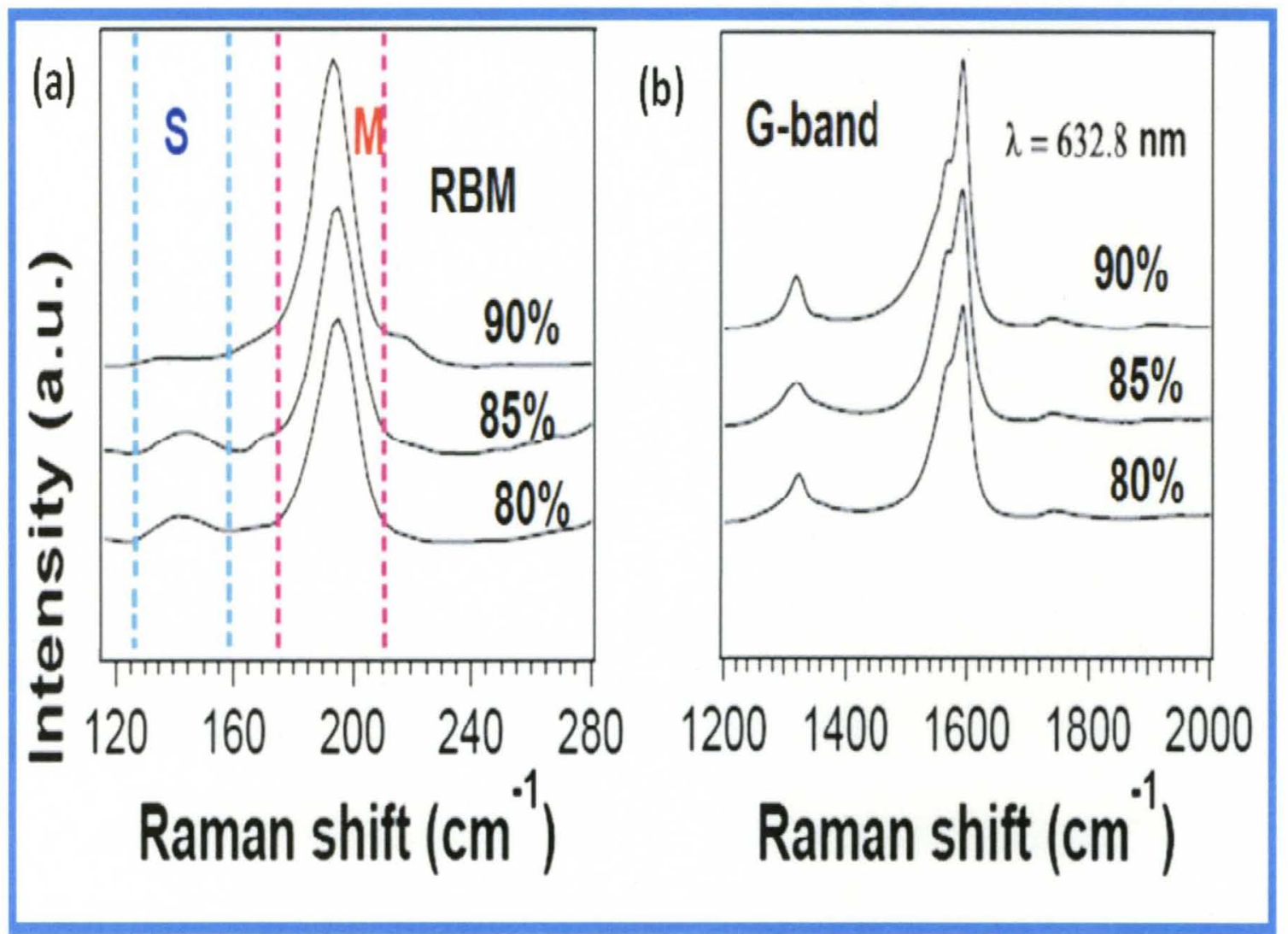

Figure 3-4: The Raman spectra of M_SWNTs. (I = 632.8-nm laser wavelength). ${ }^{96}$

The use of commercially available HiPco SWCNTs as the reference sample (37:63) ratio of metallic to semiconducting tubes was estimated based on photoluminescence measurements, which is close to those reported in at a (39:61) ratio. ${ }^{92}$ This results in a determination of about $96 \%$ metallic tube fraction in the sample with the highest $\mathrm{R}=20.2$. However, HiPco SWCNTs show a noticeably different diameter 
distribution than these samples. This may cause a large inaccuracy in this estimation, as the optical transitions are sensitive to the tube diameter. Therefore, reference samples are prepared which consist of well-dispersed individual tubes grown on the same silicon substrate under analogous conditions as the samples used in this work. 


\section{3-1-2. Production of graphene}

Graphene is a planar sheet of carbon atoms bounded together with double electron bonds $\left(\mathrm{sp}^{2}\right)$ making one atom thick film. The atoms in graphene are arranged in a honeycomb-style crystal lattice. Graphene is a basic building block for graphitic materials including carbon nanotubes. The scotch tape method is employed to isolate individual graphene planes. Graphene can be attached to a desired location for FE characterization using electrostatic deposition ${ }^{97}$ or manual brushing of nonmaterial solutions.

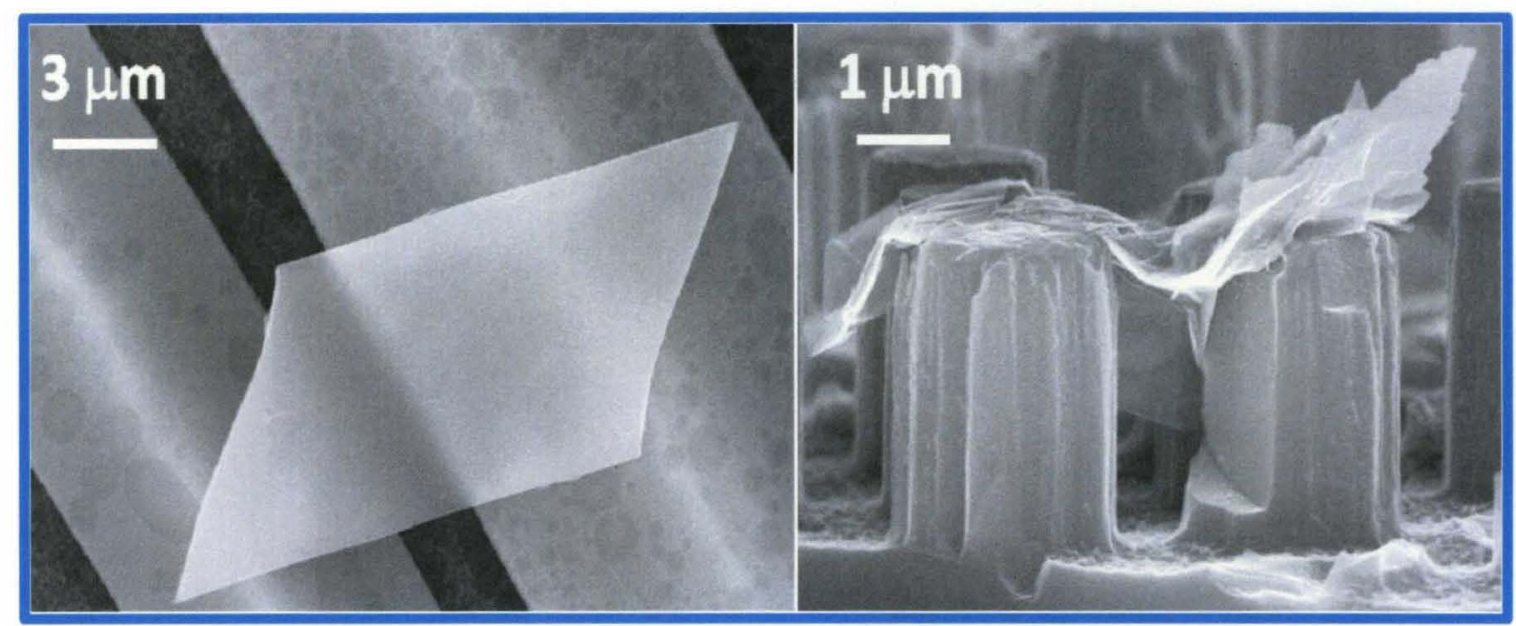

Figure 3-5: SEM picture of graphene sheets deposited on a silicon (a) trenches (b) pillars. ${ }^{98}$

Figure 3-5 is SEM images of few graphene layers deposited on a silicon trenches and pillars. The transparency of the sheets indicates the thinness of the graphene layers. The electrostatic field assists in enlivening some of the loosely bonded graphene layers from a cleaved highly pyrolitic graphite (HOPG). Monolayers up to several layers of graphene can be deposited on a desired substrate by controlling the applied voltage between 3-10 KV. The edges of the graphene sheets have two types of structures, zigzag or armchair. Zigzag structure would probably be an efficient EE sites. 


\section{3-1-3. Fabrication of nanowires}

Two types of nanowires are investigated for a possible use as cold electron emitters; e.g. gallium-silver $\left(\mathrm{Ag}_{2} \mathrm{Ga}\right)$ alloy and tungsten oxides $\left(\mathrm{WO}_{2}\right)$.

$\mathrm{Ag}_{2} \mathrm{Ga}$ nanowires form when gallium reacts with silver which at room temperature. The reaction takes place instantaneously to produce bulk nanowires. ${ }^{99}$ These kinds of nanowires are fabricated selectively on a desired location by using the method developed by Yazdanpanah et el. ${ }^{100}$ Illustration of the steps are shown on Figure 3-6 in a form of time-lapse SEM images of nanoneedle formation. ${ }^{99}$ The process is performed at or near room temperature. First the gallium (Ga) droplet is melted and, due to its strong supercooling property, it remains melted for extended periods of time at $25{ }^{\circ} \mathrm{C}$ (even though its melting point is $29.7^{\circ} \mathrm{C}$ ). The AFM tip is sputter-coated with a thin film of silver. After dipping the tip into the gallium, nanowires form in as little as a few seconds to as much as few minutes. Then, either the gallium meniscus recedes from the wire or the wire is pulled from the droplet. This process has been successfully performed using a micromanipulator while viewing the AFM tip and droplet under a scanning electron microscope (SEM), using a nanomanipulator with joystick control under a SEM, or in an AFM with limited information feedback to the operator. The aspect ratio of the formed nanowire can be controlled by room temperature monitoring and pulling speed.

The second type is tungsten oxide nanowires grown on a silicon substrate. The synthesis of $\mathrm{WO}_{2}$ consists of the chemical-vapor transport of metal oxide vapor-phase species by means of air or oxygen flow through hot filaments onto the desired substrate.

${ }^{101}$ The results show that the density of the nanowires can be varied from $10^{6}-10^{10} \mathrm{~cm}^{-2}$ by 
varying the substrate temperature (Figure 3-7). The diameter of the nanowires ranges from $20-100 \mathrm{~nm}$.
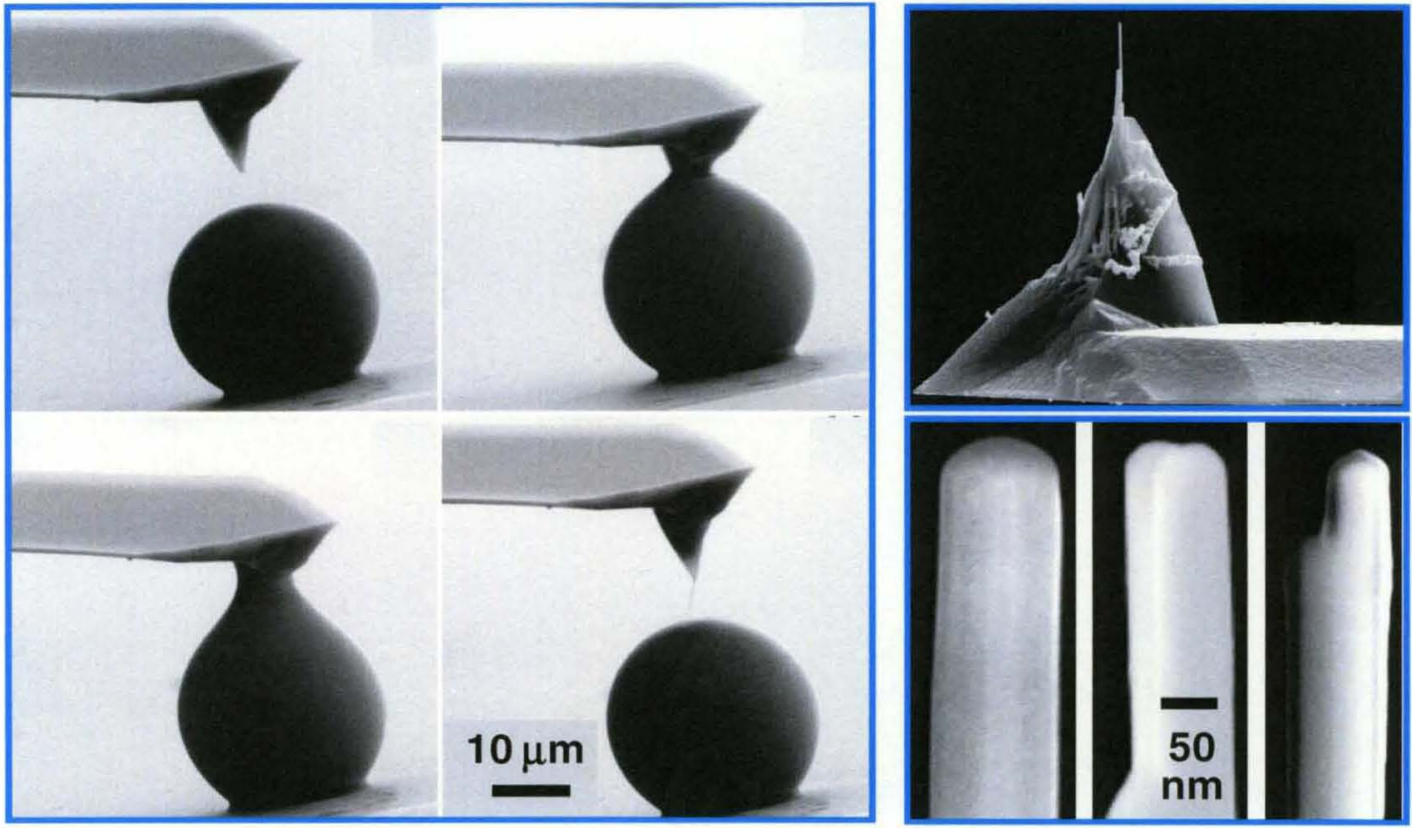

Figure 3- 6: Time-lapse SEM images of the nanoneedle formation. ${ }^{99}$

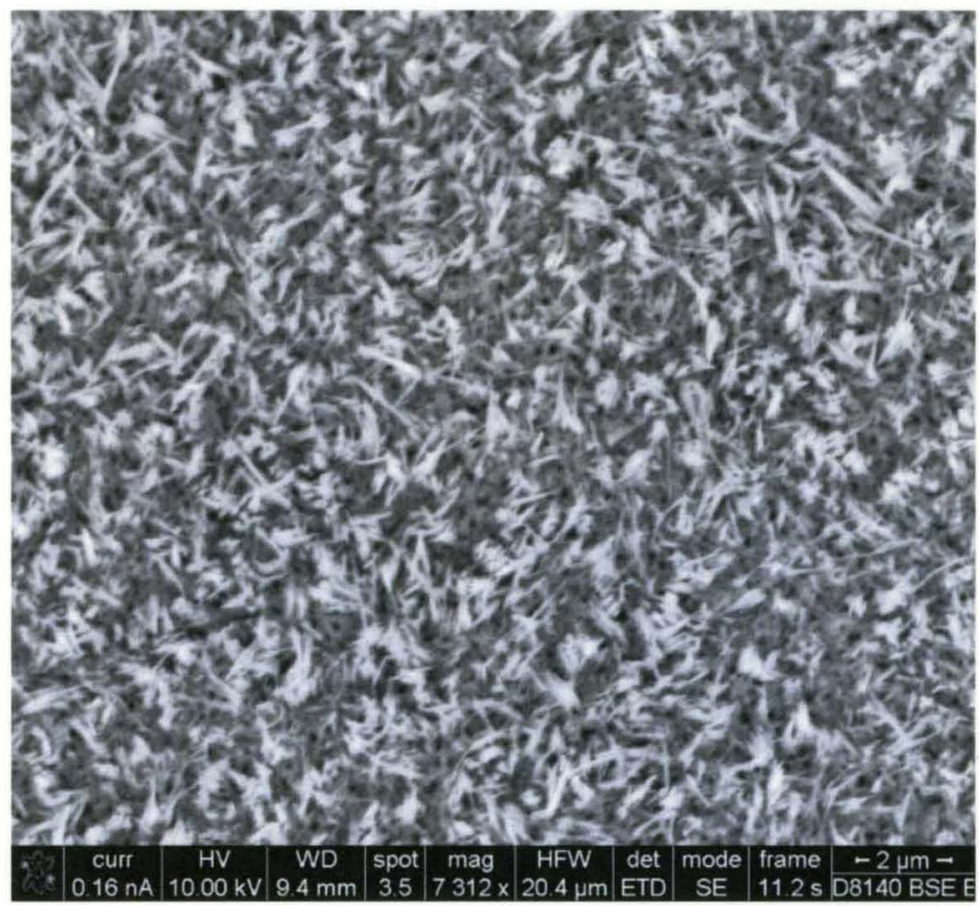

Figure 3-7: SEM image of tungsten oxide nanowires. ${ }^{101}$ 
The last type of nanowires provided for comparison is iron oxide nanowires which exhibits no detectable emission and is eliminated from further EE investigations 


\section{3-1-4. Fabrication of nanoelectrodes}

There is a need of sharp counter electrodes in order to approach and collect the emitted electrons from a desired emission site and to minimize the contribution of other

electrons from neighboring tips to the total emission current. Two main techniques of making the nano-electrodes to act as electrons collectors in the In-Situ FE experiments are demonstrated.

\section{a. Pipette pulling technique}

The first technique involve the use of the Sutter P-2000 laser based micropipette puller to make quartz nanopipettes followed by metallization using a thin film evaporation coating technique. The principle of the puller is simple. A short length of quartz glass tubing about $10 \mathrm{~cm}$ in length and $0.5 \mathrm{~mm}$ in diameter is clamped to two $\mathrm{V}$ grooved tracks. The tongs are constructed so that approximation of the handles causes the jaws to separate. An elastic band is stretched across the handles, but the jaws are prevented from separating by the capillary tubing. The tubing is locally heated at its center with a $\mathrm{CO}_{2}$ laser and the softened glass pulled to a fine capillary tip as the result of the restoring force of the elastic band. Simultaneously, with the sudden release of tension, the microflame is automatically pushed aside. Disadvantages of these nanopipettes are that they are fragile and require extra time for coating with a conductive material which is turn needs prior processing to prevent metal peeling off. 


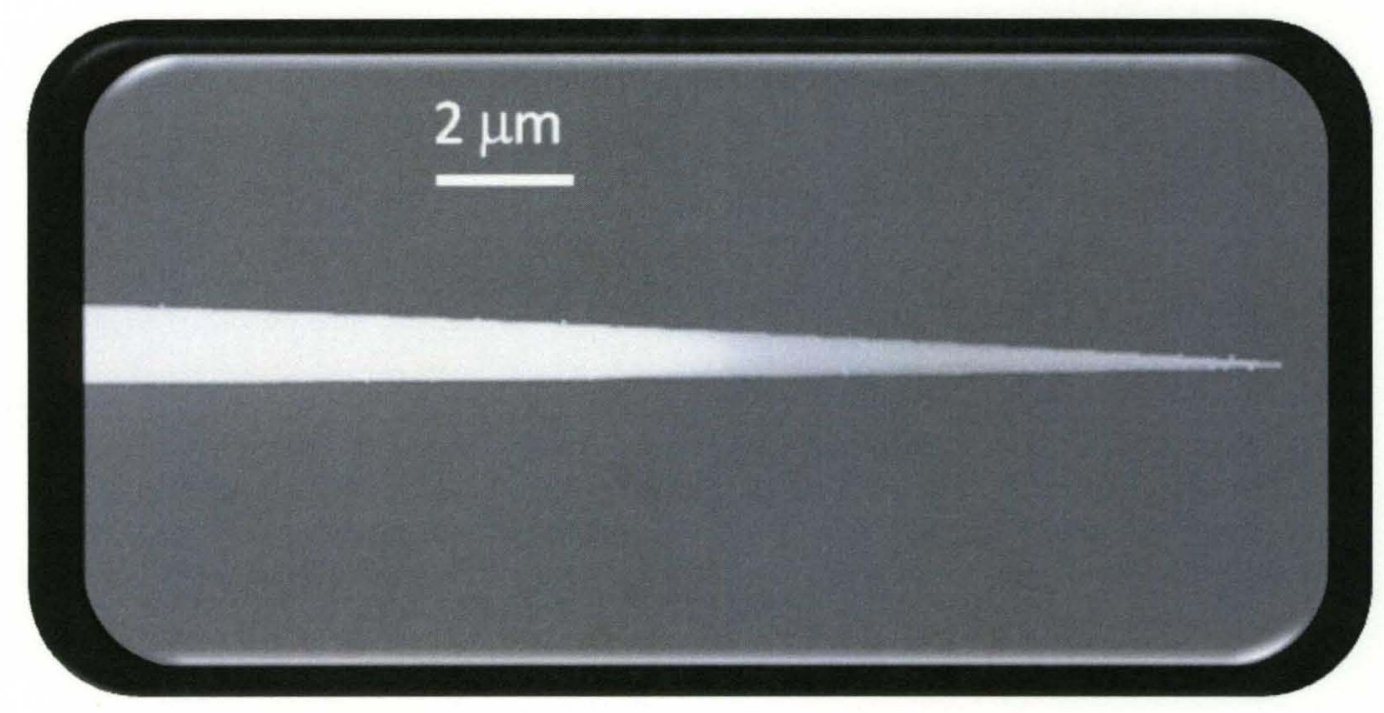

Figure 3- 8: SEM image of gold coated glass nanopipette ${ }^{102}$

\section{b. Electrochemical etching of STM tips}

The second method is the electrochemical etching of tungsten wire in a basic solution. STM tips can be prepared using several different methods. This section describes two the methods adopted in this work: (1) by cutting a wire and (2) by using an electrochemical tip etcher. A new STM tip must be prepared when first setting up for STM and also whenever the tip being used becomes damaged or oxidized.

A fast technique can be adopted to make sharp tungsten tips. A tungsten wire having diameter of $250-750 \mu \mathrm{m}$ is cut at a $45^{\circ}$ angle by means of a pair of sharp wire cutters just by gripping the free end of the wire tightly with a pair of needle-nose pliers. A sharp tip can be produced using this method tips. The disadvantage of using this technique is that the overall shape of the resulting tips is not precisely determined and several tips may appear at the cut end of the wire.

An alternative way is adopted to prepare a well defined, sharp and high aspect ratio STM tip. A $500 \mu \mathrm{m}$ diameter tungsten wire is used as the working electrode (anode) in an electrochemical cell. The counter electrode (cathode) is made of a cylindrical 
hollow graphitic block. The tungsten wire is secured within a wire-holder and positioned at the center of the counter electrode (Figure 3-8). A basic solution consisting of $2 \mathrm{M}$ of $\mathrm{KOH}$ is prepared and used as the electrolyte. Both tungsten and counter electrodes need to be partially immersed into the electrolyte which is poured into a beaker in order to close the electrical circuit for the etching current to flow through. The etching process is forced to stop at a shutoff current of 0.5 mA. Figure 3-8 displays (a) Schematic of the electrochemical cell adopted for the production of the STM probes making up the anode in an In-situ FE experiments and (b) a SEM image of the resulting electrochemically etched STM tip.

The following reaction takes place ${ }^{103}$ :

$$
\mathrm{W}+2 \mathrm{OH}^{-}+2 \mathrm{H}_{2} \mathrm{O} \quad \rightarrow \quad \mathrm{WO}_{4}{ }^{2-}+3 \mathrm{H}_{2}
$$



Figure 3-9 (a) Schematic of the electrochemical cell used to make the STM probes (b) SEM image of an electrochemically etched STM tip. ${ }^{104}$ 


\section{3-2. Experimental set-up}

A number of nanomaterials are produced in large quantity and wide variety permitting their characterization as electron point sources. The FE characterization of these nanostructures consists of studying the behavior of emitter arrays (Bulk characterization) and single emitter (In-situ characterization). Each of the bulk and In-situ investigations of nanostructures and the type of $\mathrm{EE}$ to observe require different arrangement. However, all EE measurements require that the emitters operate within vacuum environments, anode or triode configurations and vacuum-heat compatible electrical connections.

FE measurements are performed on the samples under vacuum pressure ranging from $1 \times 10^{-5}$ to $5 \times 10^{-7}$ Torr at room temperature. TE is performed at much higher temperatures up to $1500 \mathrm{~K}$ and at similar pressure conditions as FE. EE from materials

generally requires higher vacuum environments, pressure lower than $10^{-9}$ Torr needs to be achieved for better emission efficiency. A vacuum chamber meeting these requirements is under development.

\section{3-2-1. TE experiment set-up}

\section{a. Bulk Measurements}

The bulk TE characterization system is constructed using the same vacuum system utilized in the FE set-up with some additional changes. The system is outfitted with a custom made molybdenum sample holder, heater and two isolating pieces of ceramic. 


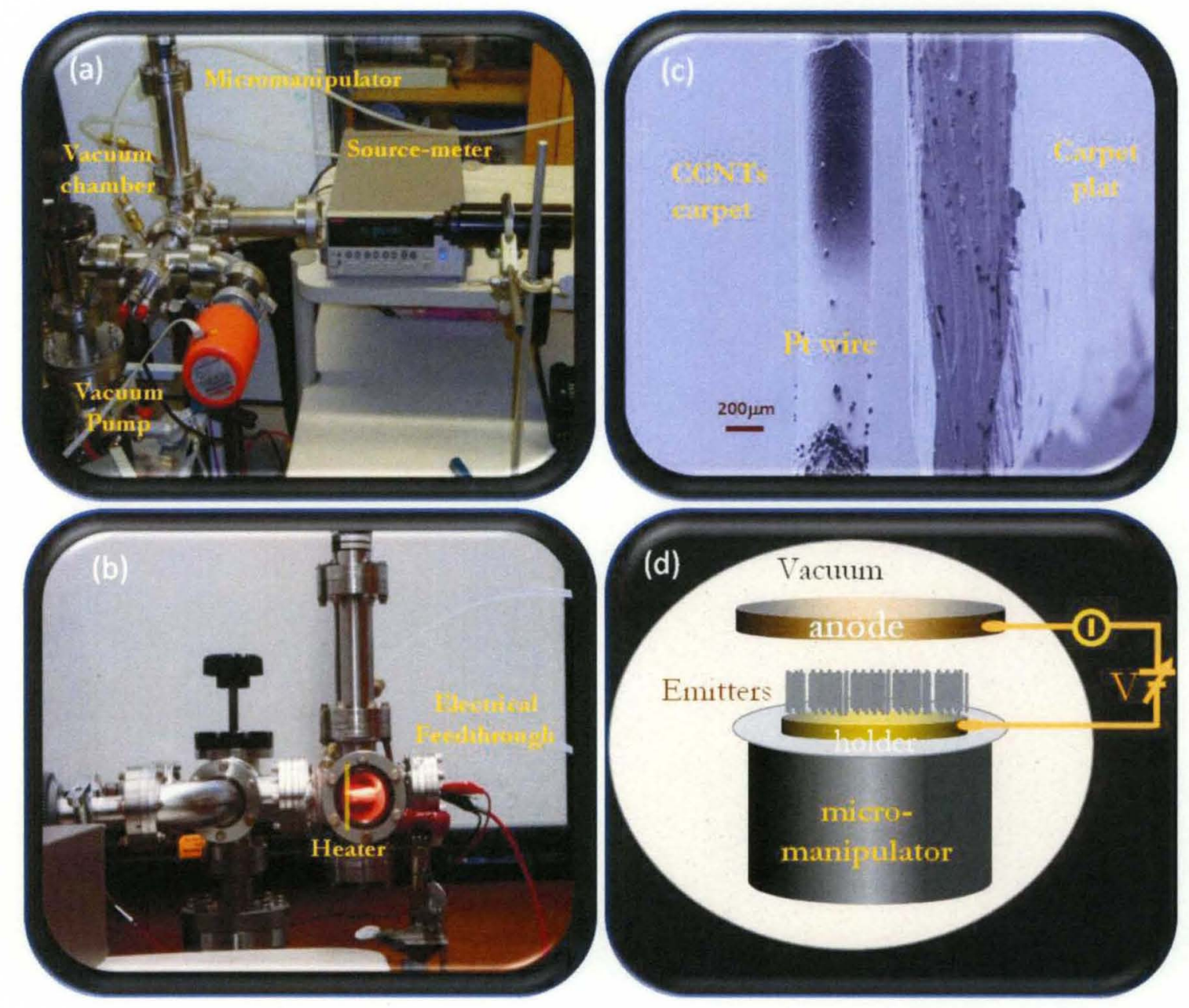

Figure 3-10: (a)Picture of the EE set-up (b) Front-view showing the glowing heater (c) SEM image of the anode-cathode (d) Schematic of the diode configuration. ${ }^{105}$

Figure 3-10 shows the system used for the bulk FE and TE characterization. It consists of the chamber, viewport the glowing heater and the optical pyrometer. The design of the sample holder used for a more stable and optimum measurements.

The sample holder is a metallic piece designed and machined to take an essential role in the FE and TE characterization. It serves two main purposes including alignment of the sample with the anode assembly and isolating the heater and electrical wires from the vacuum chamber's walls. The purpose of the first ceramic block is to insulate the heater from the sample holder while the other block serves as a cover of almost $80 \%$ of 
the heater element to reduce heat flux from reaching and increasing the temperature of the nearby anode and secures electrical connection along with the mechanical stability of the sample under tests. The electrical connection to the electrodes and heater is achieved using vacuum electrical feedthroughs and thermally isolated wires (Figure 3-9). The vacuum chamber is equipped with a glass viewport that is used as vacuum observation port and temperature measurements. An optical pyrometer instrument is set to an emissivity of 0.7 and is used to measure the temperature. Initially the optical pyrometer is used along with a K-type thermocouple for calibration/accuracy. Comparing the set of temperatures form both instruments results in identical values with minor error $\left( \pm 5^{\circ} \mathrm{C}\right)$. Temperature swept from $480{ }^{\circ} \mathrm{C}$ to $1300{ }^{\circ} \mathrm{C}$ usually in $10-20$ degrees steps.

\section{b. General requirements}

In the case of $\mathrm{TE}$, a heater capable of attaining high temperatures with small temperature steps and an electrically insulating surface is required. The heater used for TE investigation has a maximum temperature at about $1200^{\circ} \mathrm{C}$. The nuts, bolts, barrier connectors as well as any connecting metals need to have high melting temperatures and high work function in order to eliminate any significant contribution to the EE from undesired sources. The use of pyrolytic graphite and grafoil washers is essential to ensure good electrical connections and minimize stress on the heater caused by thermal expansion from the bolts and nuts used to hold the system together. 


\section{3-2-2. FE experiments}

\section{a. Bulk FE Measurements}

The characterization system is composed of a custom built vacuum chamber. The chamber has six access ports that can accommodate a variety of experimental devices, analytical instruments, viewports, feedthroughs and other accessories as needed. In addition to the turbo pump port at the base of the chamber, two other vacuum compatible feedthroughs that provide electrical connections to the electrodes, one viewport at the front of the chamber for anode-cathode alignments and observation, and one top port having vacuum compatible micromanipulator system.

Bulk FE characterization consists of placing a flat metallic electrode, which is usually larger than the anode's substrate to collect the emitted electrons, distant from the array or carpet emitters. Figure 3-10 is a schematic of the system used for FE and TE bulk characterizations. In the case of TE a heater is usually placed on the sample holder.

FE active elements i.e., MWCNTs are grown on a silicon wafer, or copper plate. CCNTs are grown on graphite foil or platinum wire. In the case of wire, a v-grooved copper plate is machined specially to be used to hold the Pt-wire substrate so that CCNTs protrude a few microns above the plate. Both sample and copper plate were placed on a custom designed sample-holder in order to outfit the custom built vacuum chamber. A precision step controller (micrometer $\sim 3.125 \mu \mathrm{m}$ step size) is used to control the movement of the counter electrode toward the FE active elements, and thus the anodecathode distance $(d)$. Zero separation $(d=0)$ is established by observing the sudden change in resistance when the anode touches the highest CCNTs. 


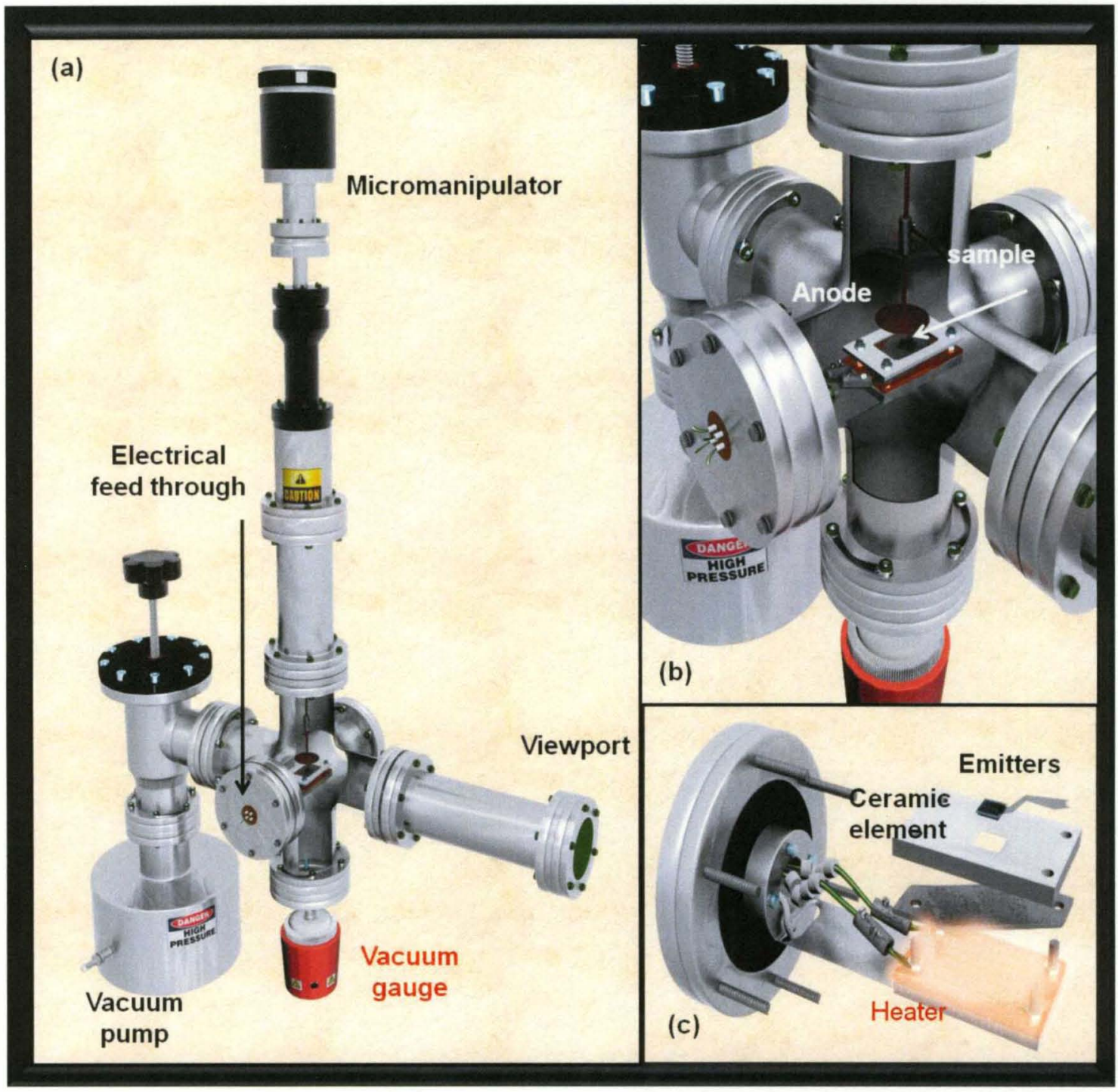

Figure 3-11: (a) Schematic of the experimental set-up used for bulk EE characterization (b) The vacuum chamber interior (c) Sample holder. ${ }^{106}$ 


\section{b. In-situ FE characterization}

A sharp STM tip is electrochemically etched to sub $10 \mathrm{~nm}$, to produce high aspect ratio structure and to approach well spaced CCNTS, MWCNTs, or the edge of graphene layers to collect the cold emitted electrons from the desired emitting sites. In the case of moderate density samples, the emission from the second-best placed tubes will not contribute significantly to the emission current since the collector is sharp enough to amass just the emitted electrons from the desired tip.

The characterization is conducted within the SEM chamber where distance (d) monitoring is secured using a Zyvex nanomanipulator system that moves freely in $(x, y, z)$ space and has coarse and fine courses with achievable precision steps as small as $2 \mathrm{~nm}$. The system has four arms each of which is equipped with a probe holder having five holes. The holes electrically connect to a breakdown electrical box. Figure 3-1 displays (a) a photograph of 4-probe manipulation system manufactured by Zyvex Nanomanipulator system equipped with four arms with 3D degrees of freedom, the inset is the low noise sample-holder for advanced electrical performance including mechanical stability. (b) is a schematic of the diode configuration used for In-situ FE characterization by the same system where just two probes are active. (c) is an SEM picture of visualizing thee diode configuration where a STM tip is brought in front of CCNT emitter within the SEM chamber.

The STM probe is secured into one of the holes using special holders to assure a mechanically stability. The probe is then brought opposite to the single emitter acting as electrons emission collector when. High resolution imaging microscopy is used in order to place the couple emitter-collector at the same $\mathrm{z}$-coordinate and within $(y, z)$ plane. 


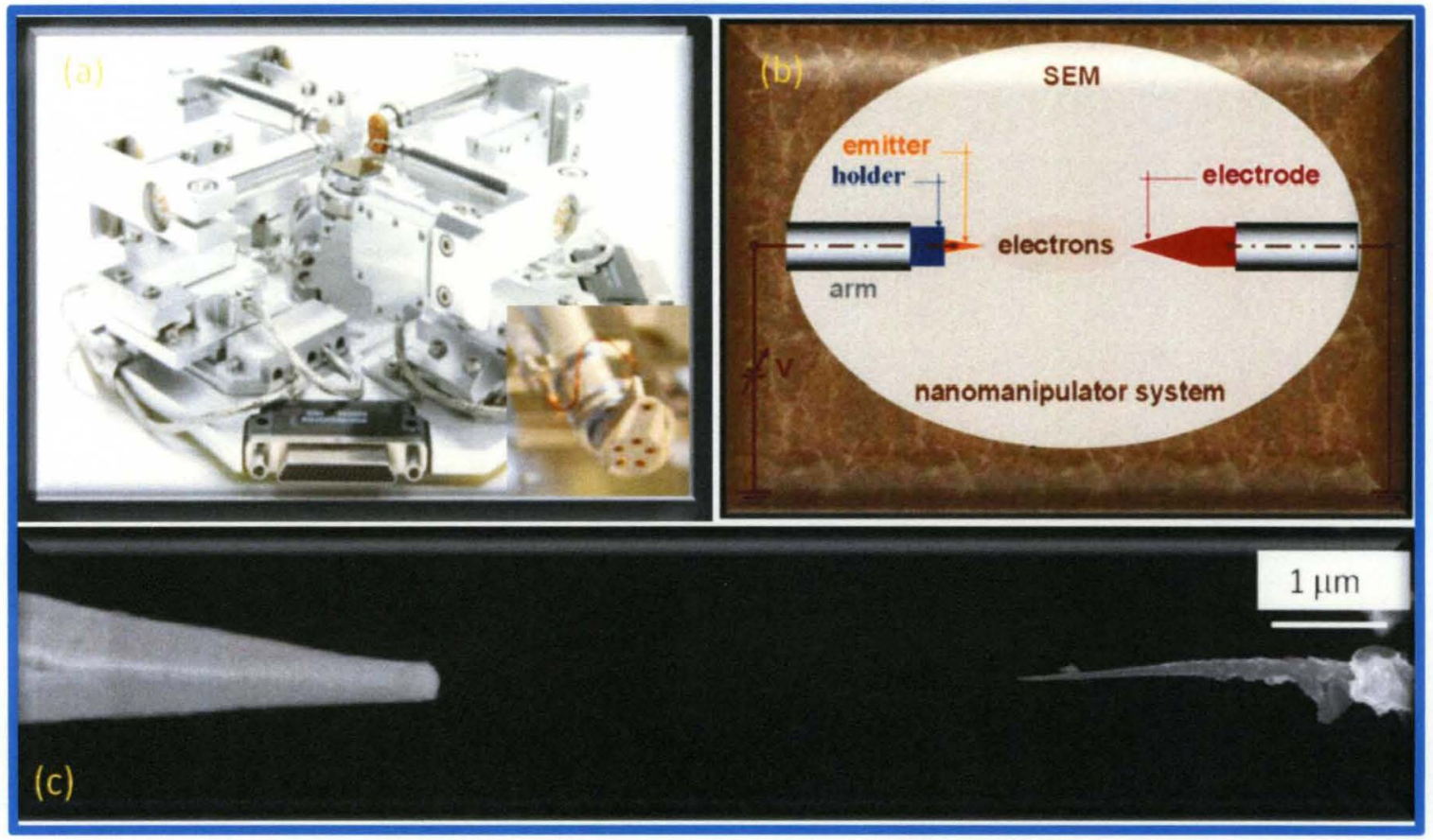

Figure 3-12: (a) Nanomanipulator system equipped with 4 arms with 3 degrees of freedom, The inset is the low noise holder for advanced electrical performance

(b) Schematic of the diode configuration used for In-situ FE characterization (c) SEM picture of STM tip in front of CCNT emitter within the SEM chamber. ${ }^{107}$

To optimize the system's performance, a new probe holder is integrated within the system to achieve low noise measurements as well as higher voltage range. The holder is outfitted with a sixth hole that is isolated from the rest and can be connected electrically directly using electrical feedthroughs. The inset of Figure 3-10 shows the shape of the low noise probe holder.

\section{c. General requirements}

In order to conduct successful FE characterization experiments high vacuum level is required. The customized system used can reach up to a pressure of $1 * 10^{-7}$ Torr, while pressure up to $1 * 10^{-6}$ Torr is usually achieved in the SEM chamber.

For each anode-cathode separation $d$ the voltage is swept from 0-500 V or 0-210 V for bulk and In-situ respectively then the current is recorded using a Keithley 6430 and 
6487 Pico-ammeter equipped with a built in variable voltage source. The emission current-voltage (I-V) characteristics are measured as a function of $d$. The obtained $\mathrm{I}-\mathrm{V}$ data are analyzed using the Fowler-Nordheim theory (Ch.II Sec.2-2). 


\section{CHAPTER IV}

\section{RESULTS AND ANALYSIS: THERMIONIC EMISSION}

In this chapter, the results and analysis of TE characterization of selected nanostructured materials are presented. These nanomaterials consist of one dimensional structures which are characterized by their interesting intrinsic properties (high aspect ratio, excellent electrical, thermal and mechanical properties). The results are outlined as follows:

- First, the EE from CCNTs, MWCNTs, M_SWCNTs, and graphene, compared to microstructures reveal significant improvement in TE.

- TE from thin films of metallic CNTs reveal superior and more stable emission than the other nanostructures.

- TE is an excellent tool to determine the work function of materials.

- The use of carbon nanostructures is characterized by the resistance against high temperature and rough vacuum environments which increase the emitters' lifetime.

- Lowering of the emitters' density had increased emission.

- The effects of the field enhancement factor and the work function on the emission are studied, as well as a discrepancy observed in the TE.

Finally, an evaluation of the parameters through which one can produce an efficient cold and hot cathode is discussed. 


\section{4-1. Thermionic emission}

In the present study direct thermionic emission measurement is not possible otherwise the use of the zero-field current density value at various temperatures is sufficient to deduce the TE properties of the emitting materials i.e. work function. The difficulty arises because thermally excited electrons tend to form an electron cloud nearby the emitting surface, giving a rise to a new potential barrier and preventing the majority of these electrons to reach the collector. Therefore a mechanism to accelerate the emitted electrons as soon as they are freed from the emitter's surface into vacuum is needed. An indirect approach of obtaining this value is adopted, for all the cathodes used, by observing the field enhanced thermionic emission (FETE) during which the barrier height and width are slightly reduced after applying an external electric field while electrons are evaporating.

The modified equation that includes the Schottky effect and governs the FETE process, called Richardson-Dushman equation, is expressed as:

$$
J=J_{0} e^{-\left(\frac{C \sqrt{E}}{K T}\right)}
$$

$J_{0}$ is referred to as the zero-field TE current density and it expressed as:

$$
J_{0}=A T^{2} e^{-\left(\frac{\Phi}{K T}\right)}
$$

Where the constant $\quad C=\sqrt{\frac{e^{3}}{4 \pi \varepsilon_{0}}}$ 
As it is seen from equation 1, the figures of merit in this case are temperature, work function and electric field. For a given material, suitable for TE, the current density increases as the electric field and/or temperature increase. In the following sections, a variation of the current versus voltage and temperature is illustrated using experimental data. These data are expressed in terms of the measured current $\left(I=J^{*} A\right)$ and applied voltage $\left(V=E^{*} d\right)$. 


\section{4-2. TE from Conical carbon nanotubes}

The CCNT samples used for the present investigations are grown on platinum wire. Figure 4-1 shows arrays of CCNTs as grown and close up view of few CCNTs having flakes at their base and sides. The CCNTs are protruding from an amorphous carbon film covering the cylindrical substrate. The growth parameters can be controlled to produce CCNTs with the desired parameters such as density and aspect ratio. CCNTs samples were used to investigate TE properties and to optimize the experimental set-up and to establish standardized method for TE as well as FETE characterization.

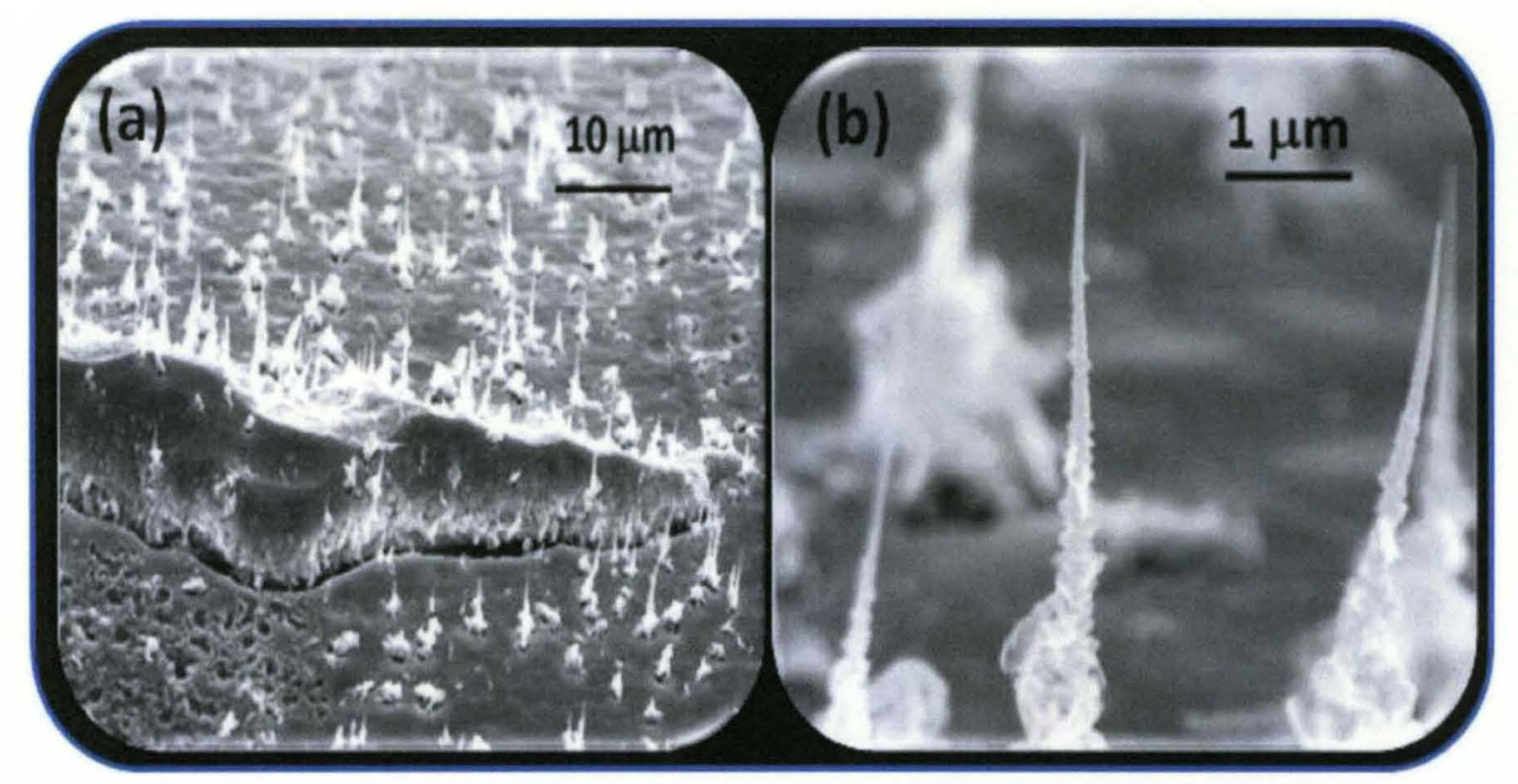

Figure 4-1: SEM images of CCNTs (a) arrays of tips (b) zoom-in image of single tips. ${ }^{108}$

Field Enhanced thermionic emission (FETE) consists of examining the combined effect of electric field along with temperature on emission from nanostructures. Three regions can generally be identified while investigating field enhanced thermionic emission from nanostructures. Each region is defined before or after a "sharp" change on the slope of the I-V curve. Figure 4-2 shows the usual behavior of CCNTs samples when 
voltage is swept from 0 to $500 \mathrm{~V}$ at temperatures ranging from 1100 to $1150{ }^{\circ} \mathrm{C}$. The current starts to increase as soon as the voltage is turned on and keeps augmenting with voltage due to the increase in the electric field present between the anode and cathode. The higher the field is, the more thermally excited emitted electrons could reach the collector. The strong electric field is able to suppress both the width and height of the potential barrier, which in turn leads to a competition between the FE and TE current.

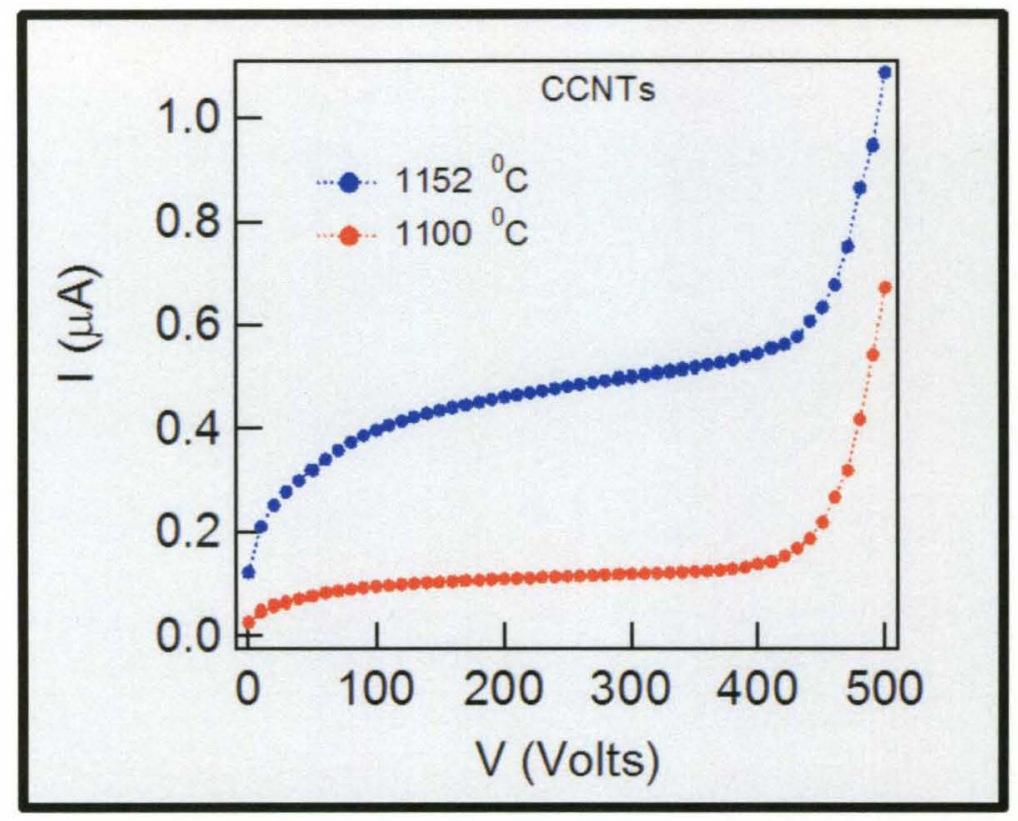

Figure 4-2: Emission current versus applied voltage of CCNTs at two different temperatures.

Next is the saturation region where most of the evaporated electrons reach the anode. The effect of temperature on emission current is apparent in this region, the higher the temperature, the higher the saturated current. The current in this region never saturates but experiences a petite positive slope as the applied field is increasing. In fact, Schottky stated that the thermionic emission entered the saturation regime at high electric fields, and the slope of I-V curves is proportional to the square root of the electric field. 
In this region, the potential barrier is reduced to produce an effective work function at which the electrons evaporation rate is quasi-constant.

The last region reveals the effect of high electric field, achieved by the local field enhancement, on the emission current behavior. The voltage swept in the present experiments reaches 1000 Volts. Consequently, the FE current appears due to the presence of an intense electric field, significantly contributing to the total current in the thermal field emission regime. Immediately, the current increases exponentially with the field and the FE becomes the predominant process of emission. The FE is mainly responsible for variations in the emission current as the ratio of FE current to TE one is more than 200. High field is observed at this point, especially when 1000 Volts is reached. This variation is illustrated in Figure 4-2.

To verify what type of emission is behind the collected current, two techniques are adopted: theoretical modeling and experimental observation of the I-V response. The electron emission theoretical modeling of the validity region for a given material is introduced in Ch.III. For a given set of electric field, field enhancement factor and work function parameters, one can predict what type of EE is predominant and estimate the outcome of the emission that is taking place. ${ }^{46}$ The second technique is based on an experimental observation where "tuning" of the anode-cathode separation is employed based on the electrical response. This is achieved by performing a room temperature FE at various anode-cathode separations. The anode-cathode distance is usually swept within the interval $12-10000 \mu \mathrm{m}$. The separation at which the field is too feeble to stimulate emission is where the TE and FETE investigations are conducted. Therefore, the main factor behind emission is heat. 
Figure 4-3 illustrates the measured current-voltage characteristic of FETE investigations of CCNTs arrays at various temperatures. The FE and thermal field emission regimes are not present in this case due to the weakness of the field. The electric field is intentionally weakened so that no contribution from FE can be observed.

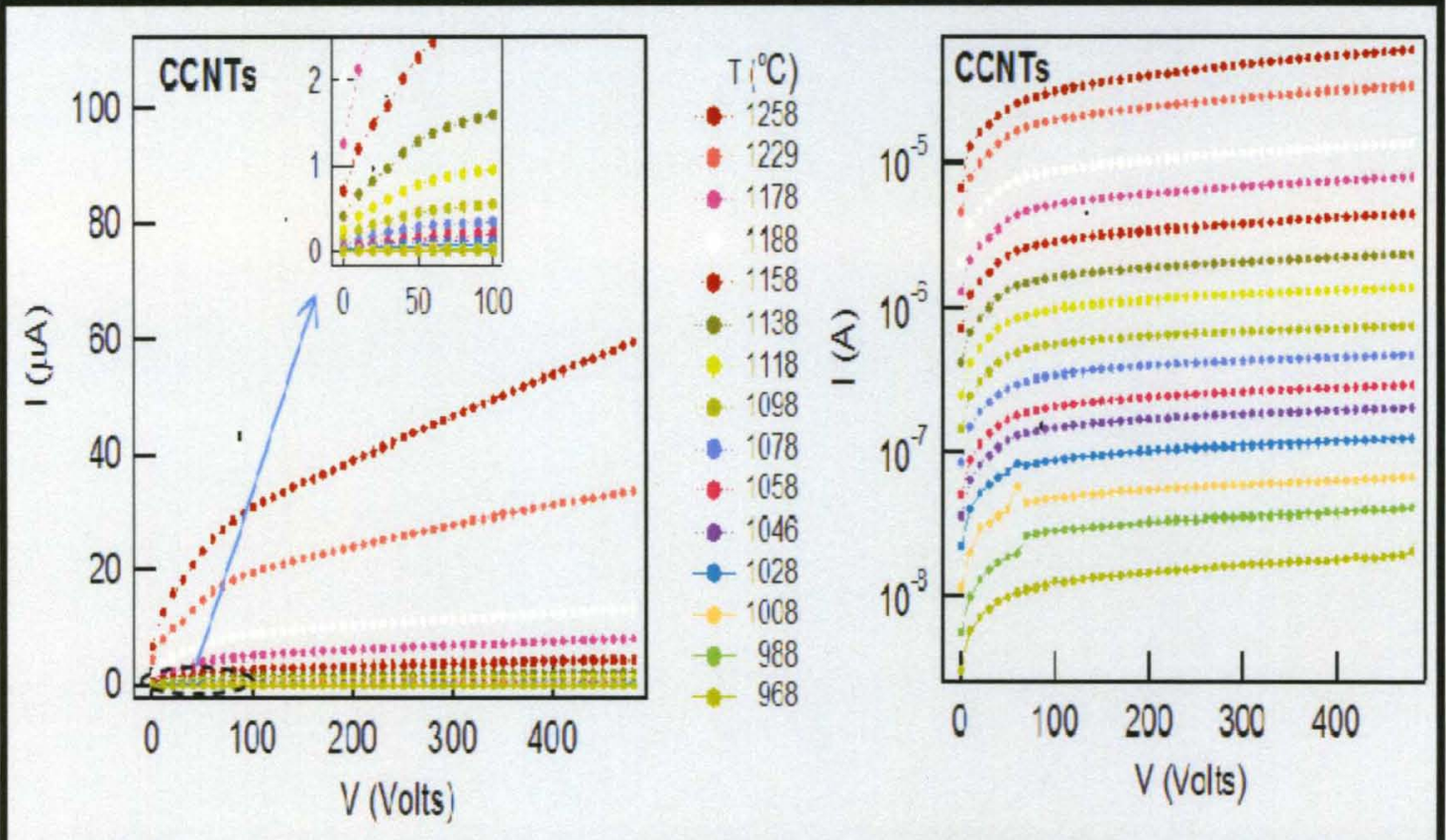

Figure 4-3: Displays the measured FETE I-V curves of the CCNTs arrays at various temperatures. (a) In linear scale, the inset is a close-up view, and (b) in Ln-linear scale.

The electric field weakness impedes the field to decrease significantly the barrier width for possible electron tunneling (emission current is comparable to the background current). A possibility of cold emission taking place in this region still exists but with insignificant contribution (FE current is a little bigger than the background current but negligible compared to the enhanced $\mathrm{TE}$ current). In this region and at higher temperatures the slope looks higher by reason of a combination of high electric field and high heat energy. As a result, a larger number of electrons have enough energy to jump over the barrier. 


\section{4-2-1. TE properties}

Once the field assisted thermionic current $I$ is measured, one needs to compute the zero-filed current $I_{0}$ by taking natural logarithm of both sides of Equation 4-1.

$$
\operatorname{Ln}(J)=\operatorname{Ln}\left(J_{0}\right)-\frac{1}{K T} \sqrt{E}
$$



Figure 4-4: TE characteristics of CCNTs (a) Natural logarithm of the emission current versus the square root of the applied voltage. (b) The Richardson plot of current versus temperature (c) The Experimental data (solid circles) and linear fitting of $\operatorname{Ln}\left(I_{0} / T^{2}\right)$ versus $(1 / \mathrm{T})$ plot.

Experimentally, the temperature dependent emission current $I(T)$ is measured as a function of the applied voltage $(V)$ at different temperatures $(T)$. From the measured data 
plotted in Figure 4-3, one can plot $\operatorname{Ln}(I)$ versus square root of $V$ as shown in Figure 4-4. By means of extrapolation, using the "saturation" region and setting $E$ to zero $(V=0)$ hence, eliminating the Schottky effect, the extrapolated current values $\left(I_{o}(T)\right)$ are derived from the intercept $\operatorname{Ln}\left(I_{0}\right)$ using:

$$
\left.\operatorname{Ln}(J)\right|_{E=0}=\operatorname{Ln}\left(J_{0}\right)
$$

Each curve of Figure 4-4(a) is used to compute the zero-field current and the corresponding temperature of emission. The resulting set of $\left(I_{0}, T\right)$ is plotted in linear scale. Plotting the Richardson plot $\operatorname{Ln}\left(I_{0}(T) / T^{2}\right) v s .(1 / T)$ should lead to a straight line. The linearity indicates that the collected electrons are due to thermionic emission from CCNTs. TE behavior is characterized by a constant slope in the Richardson plot where the slope is proportional to the work function. Figure 4-4(a) is the Richardson plot showing the variation of the zero-field TE current as a function of temperature of the CCNTs sample. Figure 4-4(b) displays the saturated emission current versus temperature plotted using Richardson analysis and its linear fitting of $\operatorname{Ln}\left(I_{0} T^{2}\right)$ versus $(1 / T)$ plot with a slope expressed as $(\Phi / K)$ (Figure $4-4(c))$.

Using the computed value for the slope from the Richardson plot, one can conclude the work function of the CCNTs. It is found that the work function of CCNTs used in this study is ranging from 3.1 to $4.2 \mathrm{eV}$. It has been reported in the literature that carbon based materials such as SWCNT, MWCNT and graphite have a work function ranging from 4.5 to $5.10 \mathrm{eV} .^{109-111}$ The difference in the work function is due either to the structure of material (i.e. single or multi-wall) or the technique employed to measure the work function. 
Ultraviolet Photoelectron Spectroscopy (UPS) is adopted as an alternative technique to measure the value of work function of CCNTs and compare it with the thermionic emission value. UPS is a technique that utilizes photo-ionization and analysis of the kinetic energy distribution of the emitted photoelectrons to study the composition and electronic state of the surface region of materials. A UPS having He-discharge lamp as a source of radiation and emitting He-I radiation of energy $21.2 \mathrm{eV}$ is used to calculate the work function of CCNTs. Figure 4-5 shows low KE slopes of the He-I spectra of the CCNTs arrays. The work function of the CCNTs is derived from the intersection of the asymptotic to the first knee of the curve of Figure 4-5 and the extrapolated background. The inset is as shown in Figure 4-5. The value of the work function measured by UPS is $4.55 \mathrm{eV}$.

Comparing the work function values obtained from TE and UPS techniques, results in $0.25 \mathrm{eV}(10 \%)$ difference. This difference can be attributed to the existence of flakes on the sides of individual pipettes, emission sites protruding out of the amorphous carbon film or ion bombardment. The occasional presence of contaminants within the vacuum chamber might be responsible for possible diffusion or chemical reactivity with nanopipettes. For better accuracy, one needs high vacuum environment plus current flushing technique to clean samples prior investigations. 




Figure 4-5: Low kinetic energy slopes of the He-I UPS spectra obtained for CCNTs. The inset displays the close-up view of the knee used to determine the work function. ${ }^{112}$ 


\section{4-2-2. Current density}

Table 4-1: displays the structural characteristics, the FETE and TE properties of the CCNTs.

\begin{tabular}{|c|c|c|c|c|c|c|c|}
\hline $\begin{array}{c}\mathrm{J}_{\mathrm{FETE} \text { max }}{ }^{* a} \\
\left(\mathrm{~mA} / \mathrm{cm}^{2}\right)\end{array}$ & $\begin{array}{c}\mathrm{J}_{\mathrm{TE} \text { max }}{ }^{\left.{ }^{*} \mathrm{~b}\right)} \\
\left(\mathrm{mA} / \mathrm{cm}^{2}\right)\end{array}$ & $\begin{array}{c}\mathrm{T}_{\mathrm{T}}{ }^{*}{ }^{\mathrm{c})} \\
\left({ }^{0} \mathrm{C}\right)\end{array}$ & $\begin{array}{c}\Phi \\
\mathrm{eV}\end{array}$ & $\begin{array}{c}\text { Density } \\
\left(\mathrm{cm}^{-2}\right)\end{array}$ & $\begin{array}{c}\text { Aspect } \\
\text { Ratio }\end{array}$ & $\begin{array}{c}\text { Radius } \\
(\mathrm{nm})\end{array}$ & $\begin{array}{c}\text { CCNTs } \\
\text { Type }\end{array}$ \\
\hline 160 & 30 & 900 & 4.2 & $\sim 2 * 10^{8}$ & 1000 & 10 & Array \\
\hline
\end{tabular}

${ }^{* a)}$ Maximum FETE current density.

*b) Maximum TE current density.

${ }^{* c)}$ Turn-on temperature corresponding to an emission current of $1 \mathrm{nA}$.

Table 4-1 displays the results of CCNTs' TE and FETE characterization as well as the properties of the conical nanotubes. From an area of $0.001 \mathrm{~cm}^{2}$, an emission current density of $160 \mathrm{~mA} / \mathrm{cm}^{2}$ and $30 \mathrm{~mA} / \mathrm{cm}^{2}$ is extracted by FETE and TE when the temperature of the substrate has reached $1258{ }^{\circ} \mathrm{C}$. The emission current density of this magnitude and at this modest temperature is among the best thermionic cathodes ever reported.

The Maximum FETE current density that is extracted from 'as grown' CCNTs is $\sim 160 \mu \mathrm{A} / \mathrm{cm}^{2}$ Obtained at $1258{ }^{0} \mathrm{C}$. Pure TE current density emitted from these emitters is approximately $30 \mu \mathrm{A} / \mathrm{cm}^{2}$. This current density value can be enhanced by optimizing the anode-cathode separation and the anode's geometry. Figure 4-6 illustrates the I-V characteristics of CCNTs investigated at $1390 \mathrm{~K}$ at two different anode-cathode separations. Comparison of the curves of Figure 4-6 demonstrates that the separation can be controlled using a micromanipulator to increase the emitted current while eliminating or reducing any meaningful contribution from the field emission. In addition, designing a 
new anode with different shape, e.g. semi-cylindrical, can lead to the collection of more emitted electrons and hence, optimize the total emitted electrons.

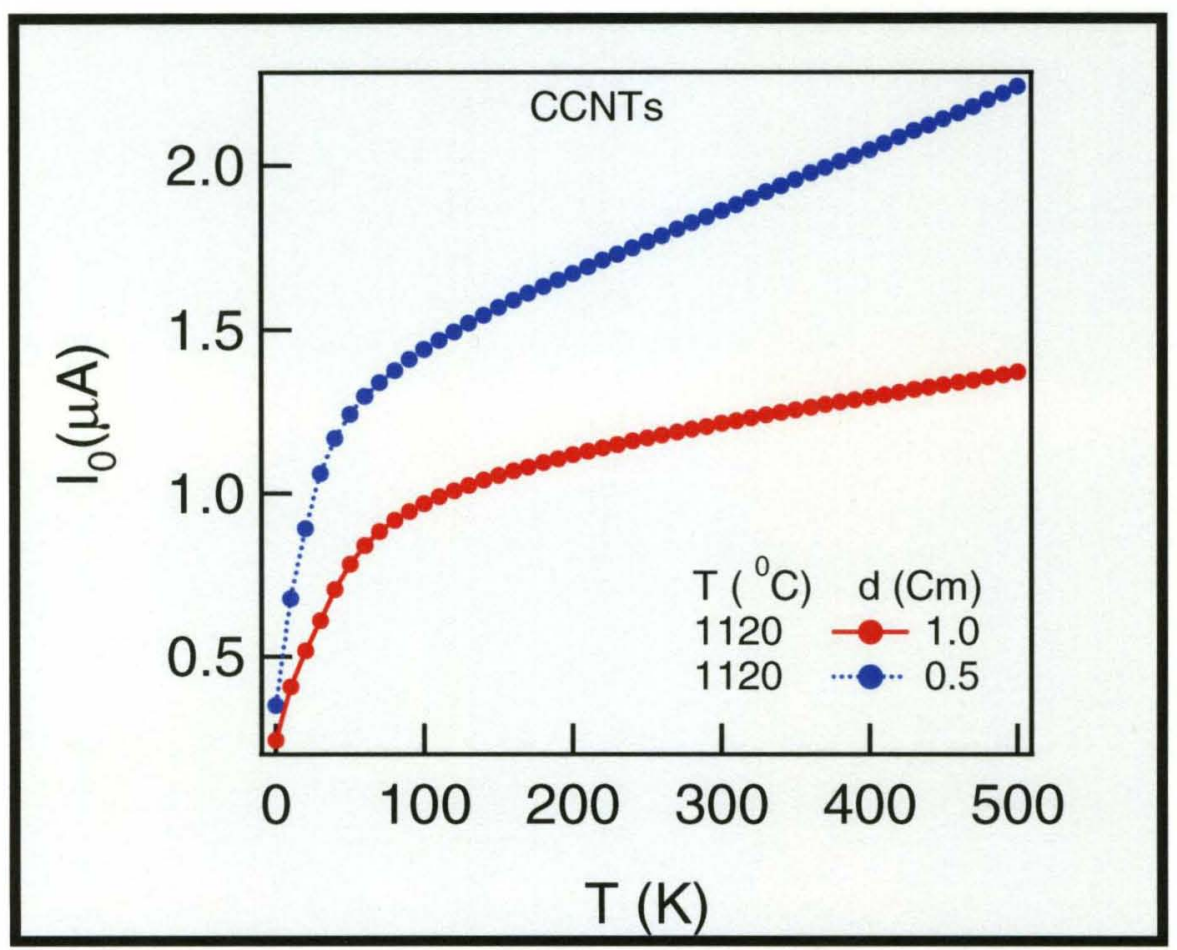

Figure 4-6: I-V characteristics of CCNTs sample at two different anode-cathode separations. 


\section{4-3. Thermionic emission from semiconducting multiwall carbon nanotubes}

MWCNTs are the next hot cathodes investigated in the present study. Figure 4-7 is SEM image of semiconducting MWCNTs used for TE measurements. The MWCNTs samples consist of vertically aligned tubes, having high density and high aspect ratio with an average tip diameter of $60 \mathrm{~nm}$.

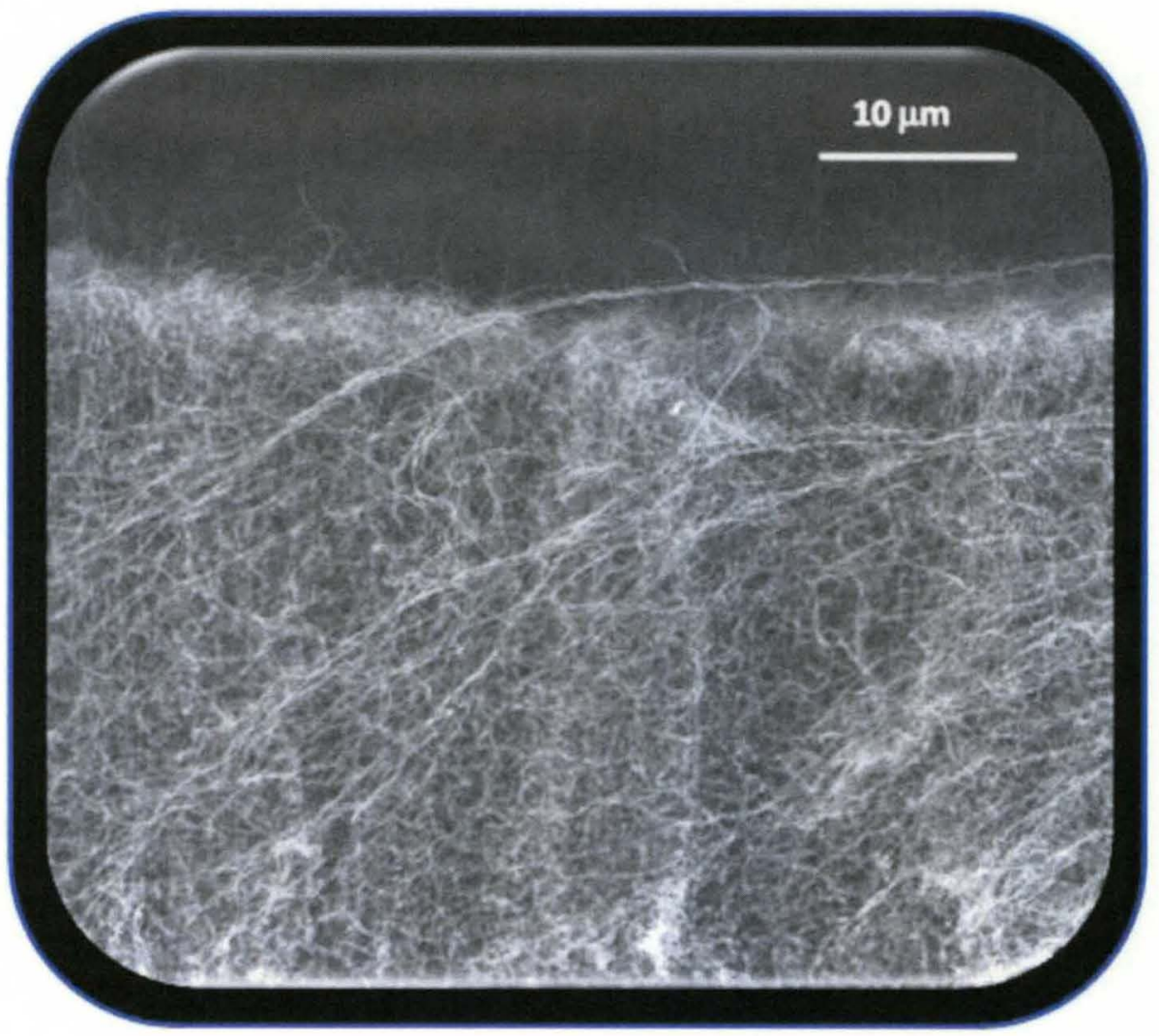

Figure 4-7: SEM images of MWCNTs. ${ }^{113}$

MWCNTs having variety of geometries and intrinsic properties including semiconducting and metallic CNTs in a temperature range of 773-1433K are investigated. They exhibit excellent $\mathrm{TE}$ emission properties as compared to the conventional TE emitters like tungsten; with lower emitting temperatures along with high melting temperature, high emission current and consistent emission characteristics. 
Two main categories of CNTs are under characterization, the first one is semiconducting MWCNTs grown by microwave plasma CVD with high density and vertical alignment. The second group is a thin film of metallic SWCNTs that are grown by CVD on Si substrate.

MWCNTs are expected to produce extremely high current density by reason of their high packing density and high aspect ratio. However, the samples used in this work produced lower current densities. This is attributed to the extremely high CNT density due to which the nanotubes are observing high field screening. Therefore very weak local field enhancement at the tips (which impede the bending of the potential barrier) is seen by the electrons. Consequently, no substantial reduction the in the work function is detected. As a result, few electrons are able to jump over the relatively high barrier by thermal excitation only. The pure TE maximum current density obtained from these samples is around $4 \mu \mathrm{A} / \mathrm{cm}^{2}$.

The experimental data obtained for MWCNTs characterization showing the FETE at different temperatures is plotted in Figure 7(a). Figure 7(b) shows the TE behavior of MWCNTs and the knee at which it turns-on. Using the linear fitting of Richardson plot, the slope of $\operatorname{Ln}\left(I_{0} / T^{2}\right)$ versus $(I / T)$ is computed. A straight line is obtained while fitting the TE experimental data according to the Richardson analysis. The linearity (Figure 7(c)) is indicative of TE of MWCNTs and the corresponding slope is -56226. Therefore, the derived work function for MWCNTs is $4.8 \pm 0.03 \mathrm{eV}$. This value is in good agreement within the published range of MWCNTs work function $(4.95 \mathrm{eV}) .{ }^{11}$ 


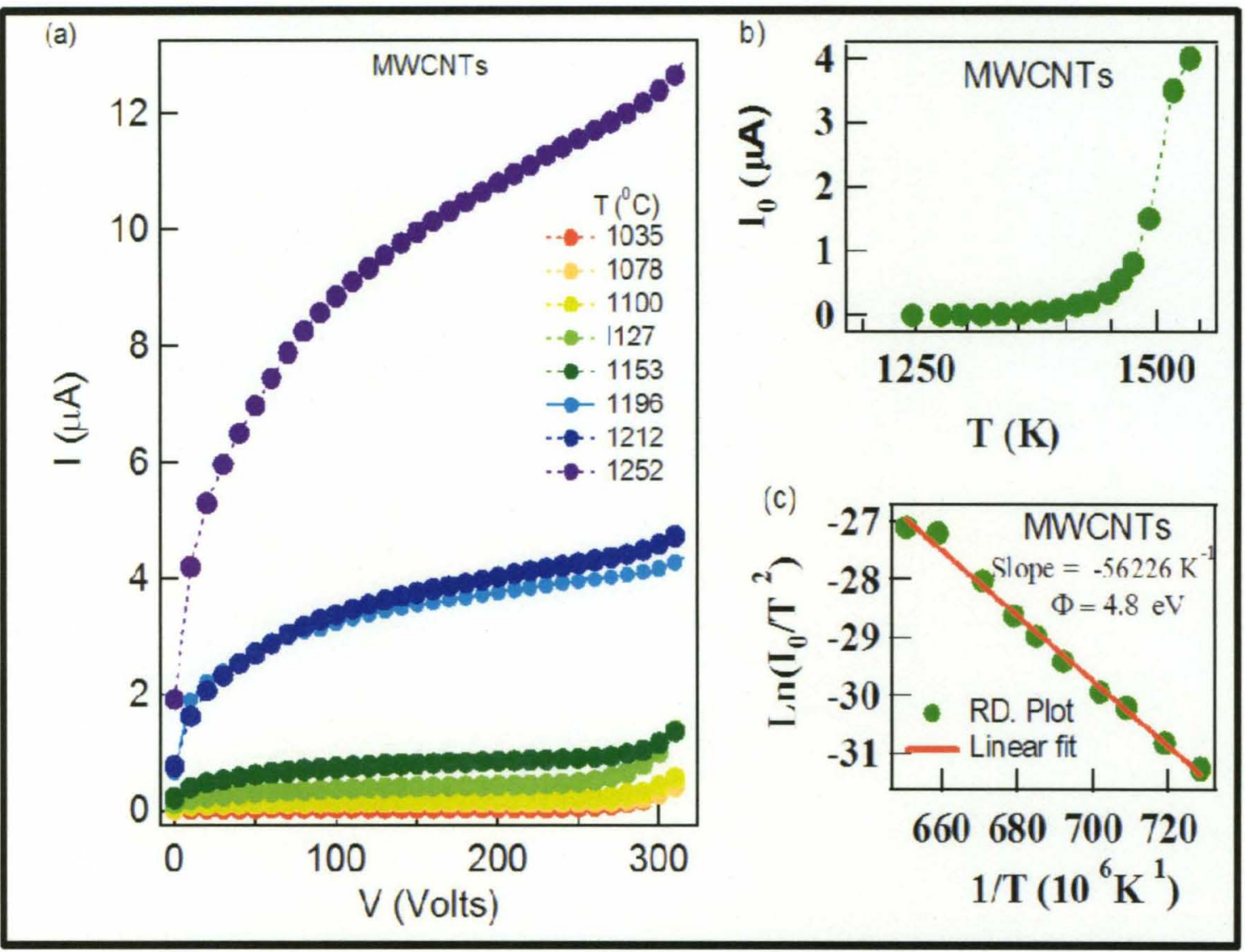

Figure 4-8: (a) Natural logarithm of the emission current versus square root of the applied voltage. TE characteristics of MWCNTs sample (b) The Richardson plot (c) The Experimental data (squares) and linear fitting of $\operatorname{Ln}\left(I_{0} / T^{2}\right)$ versus $(1 / T)$ plot.

Table 4-2: The structural characteristics, and the FETE and TE properties of the MWCNTs carpet sample used in this study.

\begin{tabular}{|c|c|c|c|c|c|c|c|}
\hline $\begin{array}{c}\mathbf{J}_{\text {FETE }} \\
\left(\mu \mathrm{A} / \mathbf{c m}^{2}\right)\end{array}$ & $\begin{array}{c}\mathbf{J}_{\mathrm{TE}} \\
\left(\mu \mathrm{A} / \mathrm{cm}^{2}\right)\end{array}$ & $\begin{array}{c}\mathbf{T}_{\mathrm{T}} \\
\left({ }^{0} \mathbf{C}\right)\end{array}$ & $\begin{array}{c}\boldsymbol{\Phi} \\
( \pm \mathbf{0 . 0 3 e V})\end{array}$ & Density & $\begin{array}{c}\text { Area } \\
\left(\mathbf{c m}^{2}\right)\end{array}$ & $\begin{array}{c}\text { Aspect } \\
\text { Ratio }\end{array}$ & $\begin{array}{c}\text { Radius } \\
(\mathbf{n m})\end{array}$ \\
\hline 13 & 4 & 1000 & 4.8 & $9 * 10^{9}$ & 1 & 10000 & 60 \\
\hline
\end{tabular}


Table 4-2 shows the results obtained after MWCNTs' TE and FETE characterization along with their properties. Both TE and FETE current densities are much lower than those obtained from CCNTs - even though their emitting area is considerably larger. The temperature of emission at which MWCNTs produce $4 \mu \mathrm{A} / \mathrm{cm}^{2}$ is $1250{ }^{\circ} \mathrm{C}$ which is relatively higher. The poorer TE properties of MWCNTs can be attributed to different factors. The most obvious one is the higher work function of MWCNTs. For macroscopic material with a work function of $4.8 \mathrm{eV}$ to produce significant current density, a temperature higher than $2000{ }^{\circ} \mathrm{C}$ needs to be reached (chapter 2). In contrast, for nanomaterials having a high aspect ratio of 10000 (Which is the case here), field enhancement should contribute to the lowering of the effective work function increasing the current density and improving the TE properties. High emitters' density and low field screening effect is accounted for such weak TE properties. This type of sample reveals the effect of the dense emitters on the overall performance of nanoemitters.

Different techniques have been adopted based on different physical causes to measure the work function of solid surfaces; resulting in a discrepancy in obtained values for MWCNTs. Surface conditions such as cleanliness and chemical reactivity with contaminants can justify this difference. TE of electrons is one of the preferred methods that use the experimental TE I-V curves to compute the value for work function. This process is preferred among a good number of groups due to its simplicity and exigency of removing amorphous carbon and other contaminates by thermal annealing. ${ }^{52}$. 


\section{4-4. Thermionic emission from Metallic SWCNTS}

Investigations and search for suitable nanostructured materials lead to the next candidates of hot cathodes by which promising results of FE and TE are developed and reported in the present study. At the present time, no reported work has indicated that the FE, TE or FETE characterization of M_SWCNTs. Further investigations of these novel nanostructures such as energy power spectroscopy, electrical and thermal properties need to be perceived in order to reveal other causes of this excellent and par suite lead to a new generation of nanoemitters.

Figure 4-9 shows a thin film of M_CNTs that are laying on a silicon substrate, grown by CVD and found to be single walled tubes. In M_CNTs with their metallic properties can be an excellent EE based device.

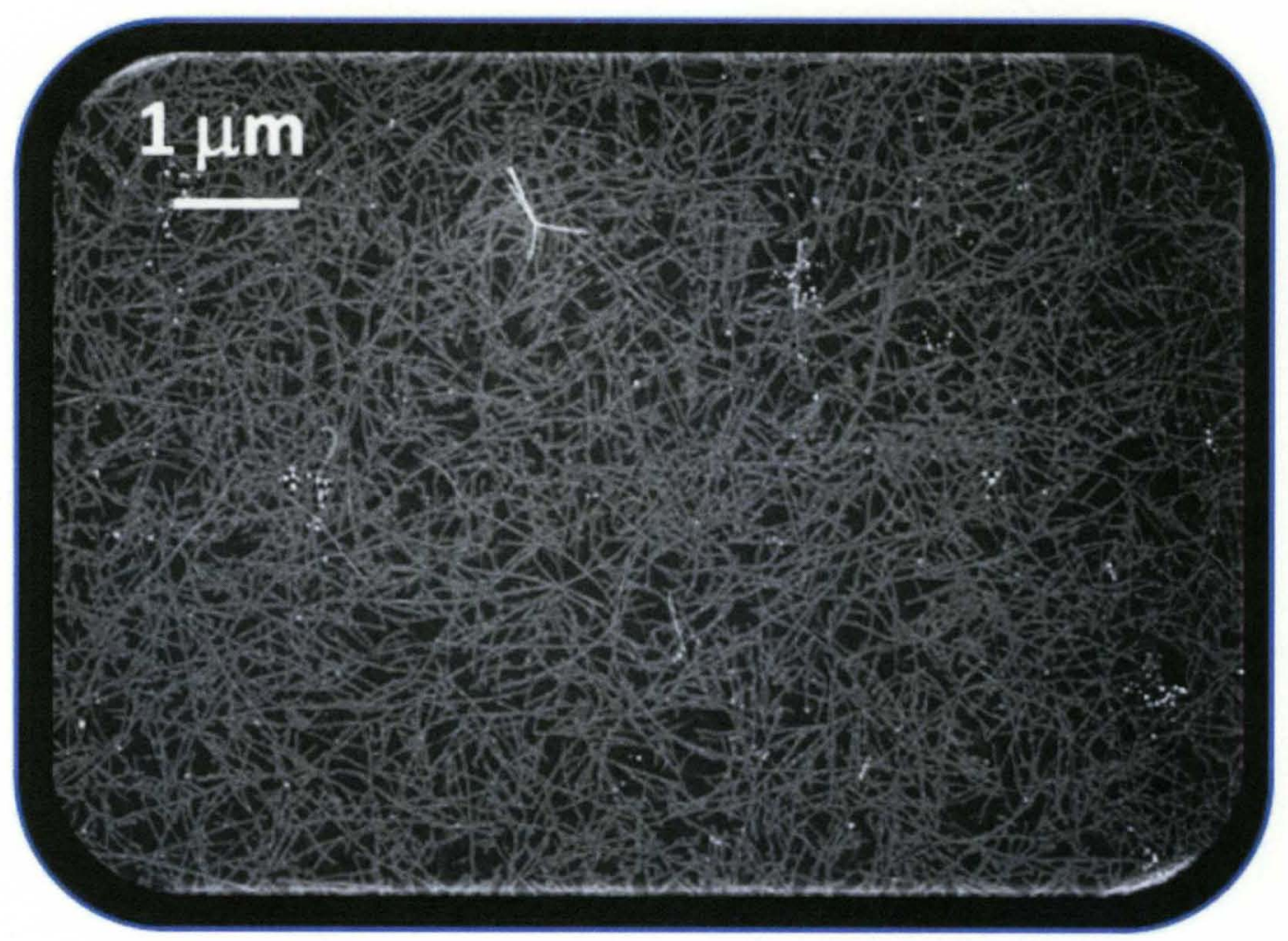

Figure 4-9: SEM image of M_SWCNTs grown by CVD system. ${ }^{114}$ 
The samples used consist of a dispersed film containing SWCNTs. The SWCNTs have an average diameter of 1-2 $\mathrm{nm}$ and length of $10 \mu \mathrm{m}$. The Raman spectroscopy shows that $\sim 96 \%$ of the tubes are metallic.

Figure 4-10 presents the FETE current versus the applied voltage for M_SWCNTs. During this characterization the voltage is swept up to 1000 Volts and leads to the appearance of a short saturation region that looks like a step at 200 Volts and starts to shift towards the $\mathrm{Y}$-axis as the temperature increases. Figure 4-9 illustrates this observation as the $\mathrm{x}$-axis is plotted in Ln-scale.
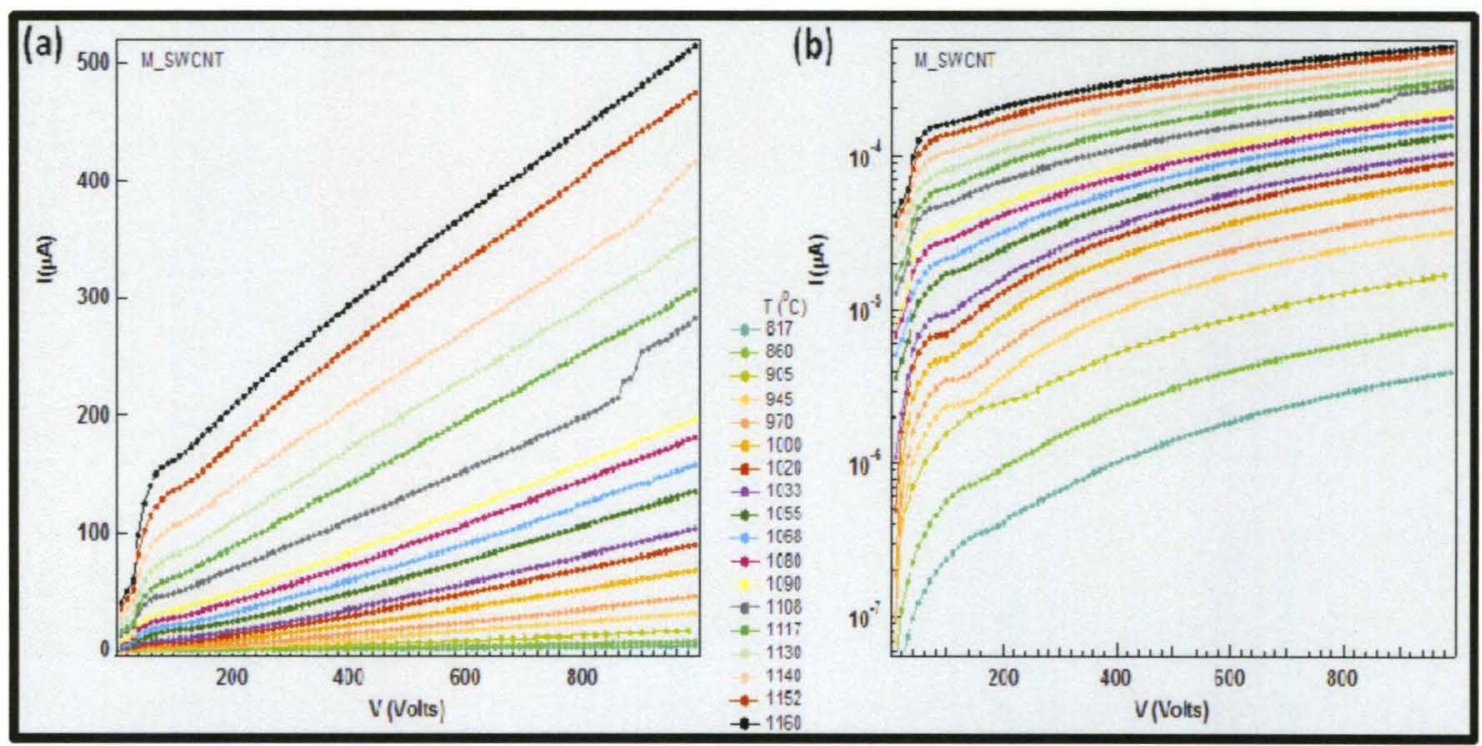

Figure 4-10: The measured FETE I-V curves of the M_SWCNTs arrays at various temperatures. (a) In linear scale, the inset is close-up view and (b) in semi-Ln scale. ${ }^{115}$

M_SWCNTs exhibit good EE properties starting with the FE regime that starts to contribute to the emission at about 200 Volts corresponding to a field of 1.1 Volts/ $\mu \mathrm{m}$ at $350 \mathrm{~mm}$ anode-cathode separation, then a maximum FETE and zero-field TE current densities of $0.5 \mathrm{~mA} / \mathrm{cm}^{2}$ and $100 \mu \mathrm{A} / \mathrm{cm}^{2}$ respectively. In addition, the emission starts 
turning on at temperatures as low as $620{ }^{\circ} \mathrm{C} .1 \mu \mathrm{A}$ is easily achievable by heating the substrate to $1000{ }^{0} \mathrm{C}$. The Work function of $\sim 4 \mathrm{eV}$ is calculated for M_SWCNTs. Since M_SWCNTs are embedded in a thin film without geometrical advantages, they are expected to have very low field enhancement. However, FE starts to contribute to the EE at early voltages.

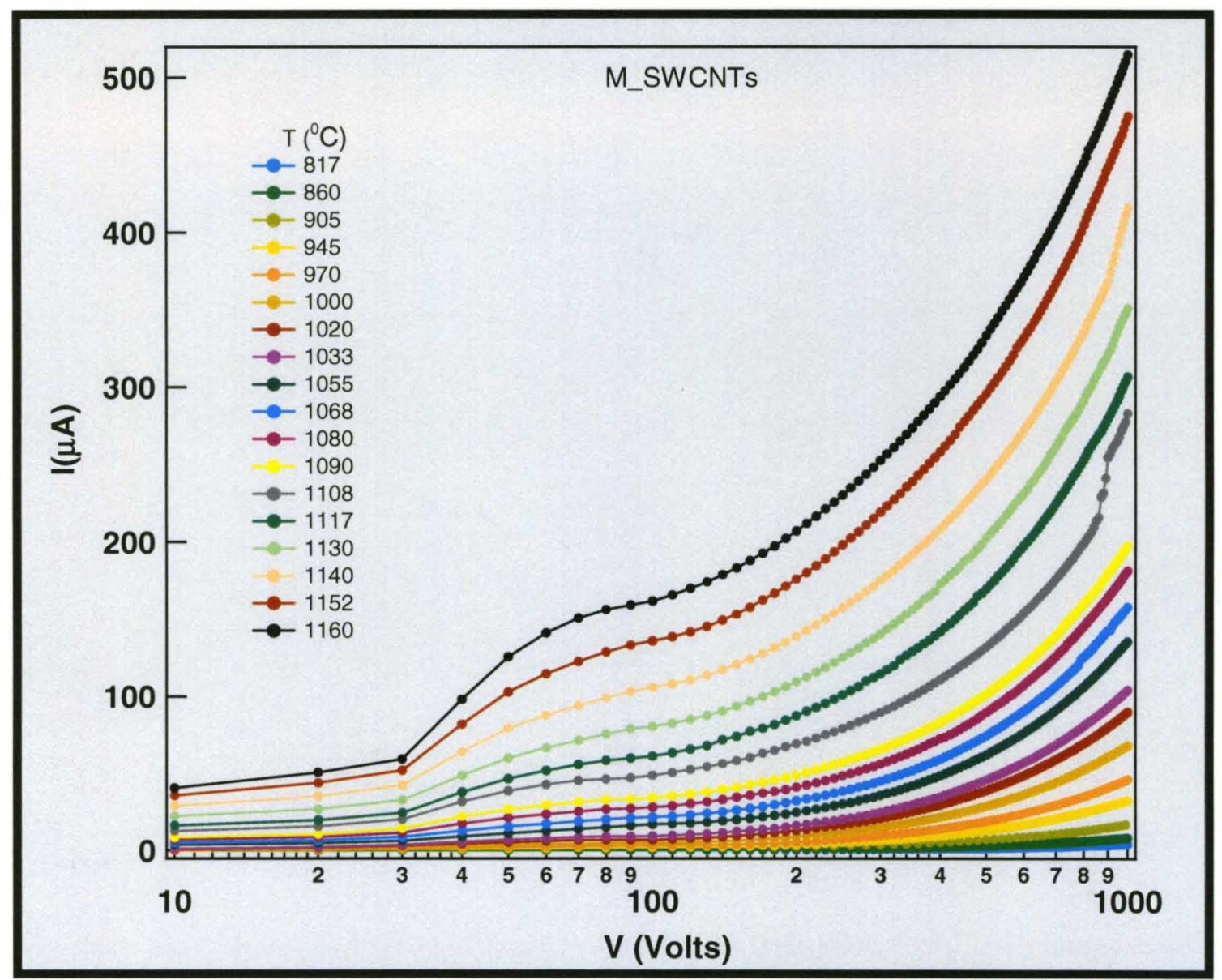

Figure 4-11: displays the measured FETE I-V curves of the M_SWCNTs arrays at various temperatures in linear-Ln scale. ${ }^{115}$

The results of FETE from M_SWCNTs are also analyzed using the Richardson equation and plot. The results are plotted in Figure 4-11. The influence of the field and 
temperature on the thermionic emitted electrons is evident through the competition between the field and temperature within the interval [100, 1000 Volts].
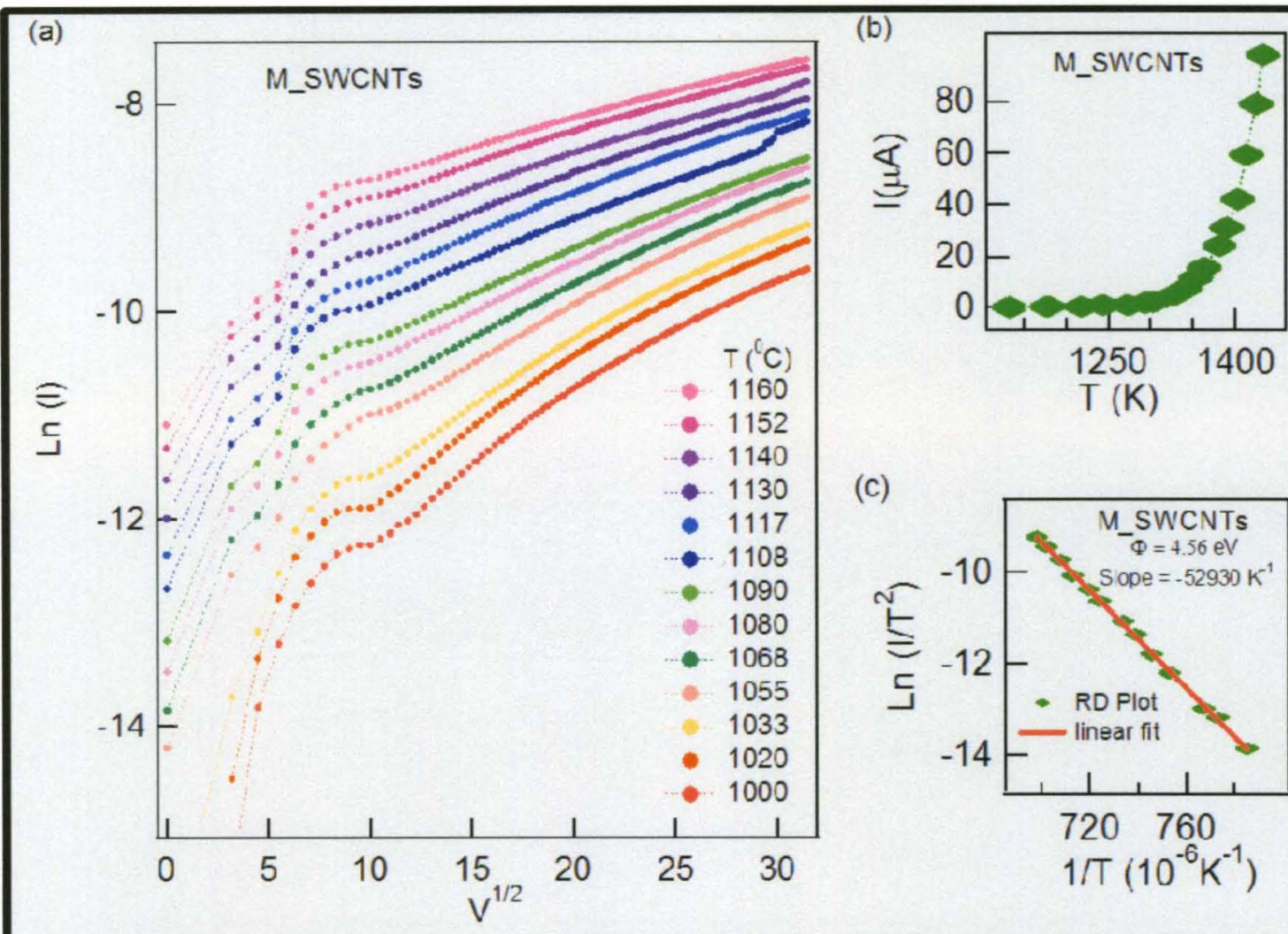

(c)

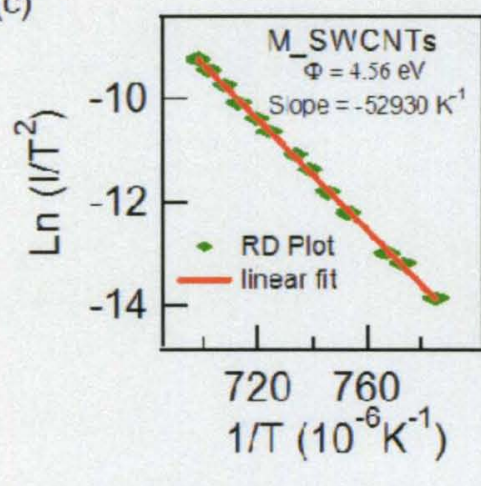

Figure 4-12: (a) Natural logarithm of the emission current versus square root of the applied voltage. TE characteristics of M_SWCNTs sample (b) The Richardson plot (c) The Experimental data (squares) and linear fitting of $\operatorname{Ln}\left(I_{0} / T^{2}\right)$ versus $(1 / T)$ plot. $^{115}$

Table 4- 3: The structural characteristics, and the FETE and TE properties of the M_SWCNTs film sample used in this study.

\begin{tabular}{|c|c|c|c|c|c|c|c|}
\hline $\begin{array}{c}\mathbf{J}_{\mathrm{FETE}} \\
\left(\mu \mathrm{A} / \mathrm{cm}^{2}\right)\end{array}$ & $\begin{array}{c}\mathbf{J}_{\mathrm{TE}} \\
\left(\mu \mathrm{A} / \mathrm{cm}^{2}\right)\end{array}$ & $\begin{array}{c}\mathbf{T}_{\mathrm{T}} \\
\left({ }^{0} \mathbf{C}\right)\end{array}$ & $\begin{array}{c}\Phi \\
(\mathrm{eV})\end{array}$ & $\begin{array}{c}\text { Density } \\
\left(\mathrm{cm}^{-2}\right)\end{array}$ & $\begin{array}{c}\text { Area } \\
\left(\mathrm{cm}^{2}\right)\end{array}$ & $\begin{array}{c}\text { Aspect } \\
\text { Ratio }\end{array}$ & $\begin{array}{c}\text { Radius } \\
(\mathbf{n m})\end{array}$ \\
\hline 515 & 100 & 500 & 4.56 & $10^{9}$ & 1 & $>1000$ & 10 \\
\hline
\end{tabular}


As grown M_SWCNTs exhibit good TE properties compared to the other structures evaluated in the present study, starting with onset temperatures as low as 500 ${ }^{0} \mathrm{C}$, maximum current density higher than $0.5 \mathrm{~mA} / \mathrm{cm}^{2}$ and work function of $\sim 4.56 \mathrm{eV}$. 


\section{4-5. Comparison}

Table 4-4 summarizes the resulting structural characteristics, the FETE and TE properties of the three CBNSs that are morphologically dissimilar and have different densities and aspect ratios.

CCNTs produce the highest FETE and TE current which reflects their lowest work function and the tendency of electrons to jump over the potential barrier present at the vacuum interface. The CCNTs moderate emitters' density reduces the field screening effect and accounts for the lowering of the effective work function.

Although the emission currents from M_SWCNTs are not as high as those from

CCNTs, they have the lowest onset temperature $\left(500{ }^{\circ} \mathrm{C}\right)$. This may be due to the metallic behavior of the M_SWCNTs and low field screening effect and maybe high thermal conductivity. The low emission current could be explained by the transport within the film which may reduce the anticipated emission current. However, the metallic behavior can cause the tubes as well as low field screening effect and maybe high thermal conductivity. The fact that M_SWCNTs are laying laterally may have increased the surface area for TE and enhance emission. If this is the case, M_SWCNTs may become potential candidates as electrons point sources.

On the other hand, EE from MWCNTs is the poorest regardless of the high density, high aspect ratio and larger emitting surface. In fact, dense emitters having work function of $4.8 \mathrm{eV}$ are expected not to have good emission at temperatures less than 2000 ${ }^{\circ} \mathrm{C}$. 
Table 4- 4: A Comparison of the TE and FETE properties and the structural characteristics of CBNs.

\begin{tabular}{c|c|c|c|c|c|c|c|c|c|c}
\hline Material & $\begin{array}{c}\mathrm{J}_{\text {FETE }} \\
\mu \mathrm{A} / \mathrm{cm}^{2}\end{array}$ & $\begin{array}{c}\mathrm{J}_{\mathrm{TE}} \\
\mu \mathrm{A} / \mathrm{cm}^{2}\end{array}$ & $\begin{array}{c}\mathrm{T}_{\mathrm{T}} \\
{ }^{\circ} \mathrm{C}\end{array} \begin{array}{c}\Phi \\
\pm 0.03 \mathrm{eV}\end{array}$ & $\begin{array}{c}\text { Density } \\
10^{\circ} \mathrm{cm}^{-2}\end{array}$ & $\begin{array}{c}\text { Area } \\
\mathrm{cm}^{2}\end{array}$ & $\begin{array}{c}\text { Aspect } \\
\text { Ratio }\end{array}$ & $\begin{array}{c}\text { Radius } \\
\mathrm{nm}\end{array}$ & Type \\
\hline CCNTs & 160 & 30 & 900 & 4.2 & $\sim 0.2$ & 0.001 & 1000 & 10 & carpet \\
\hline MWCNTs & 13 & 4 & 1000 & 4.8 & $\sim 9$ & 1 & $>10000$ & 60 & carpet \\
\hline M_SWCNTs & 515 & 100 & 500 & 4.56 & $\sim 1$ & 1 & $>10000$ & 10 & film \\
\hline
\end{tabular}

In addition to SWCNTs and MWCNTs, CCNTs and M_SWCNTs are considered as novel cathodes and could become the new generation of nanoemitters of VNE devices. Further surface treatment can improve EE properties and efficiency.

More investigations such as energy power spectroscopy, electrical and thermal properties need to be undertaken in order to reveal causes of excellent EE of M_SWCNTs. 


\section{CHAPTER V}

\section{RESULTS AND ANALYSIS: FIELD EMISSION}

In this chapter, the results and analysis of $\mathrm{EE}$ characterization of selected nanostructured materials are presented. These nanomaterials consist of one and two dimensional structures which are characterized by their interesting intrinsic properties (nano-size, aspect ratio, electrical, thermal and mechanical properties). Several results are outlined as follows:

- First, the FE from CCNTs, MWCNTs, M_ SWCNTs, and graphene, compared to microstructures and sub 100 nanometers structures reveal significant FE improvement due to:

a) The small size of the emitting sites resulted in high packing density and significant current density increase.

b) High local field enhancement: control of the growth in the form of alignment and emitters' density resulted in the reduction of field screening effect as well as optimization of the field emission proprieties.

c) Field enhancement lowering of the work function resulted in low emission turn-on temperatures and higher current density even from $5 \mathrm{eV}$ work function materials. 
- The use of carbon nanostructures is characterized by the resistance against rough vacuum environment which in turn can extend the lifetime of the nanoemitters.

- Lowering of the emitters' density with respect to their height had increased emission.

- Next, the comparison of the emission from different types of CNTs to the emission from nanowires illustrates the impact of the increased field enhancement factor.

- Lastly, the correlation between the CCNTs morphology and emission, in which FE from different lengths and densities are compared.

The effects of the field enhancement factor and the work function on the emission are studied, as well as a discrepancy observed in some of the FE results.

Finally, an evaluation of the parameters through which one can produce an efficient cold electron emitter is discussed. 


\section{5-1. FE characterization of tungsten oxide nanowires}

Tungsten oxide nanowires are characterized by reason of their extensive use as active elements of FE and TE based devices, moreover, a comparison between metallic based nanostructures with $\mathrm{CBNs}$ is needed for evaluation purposes.

A sample with an area of $1.5 \mathrm{~cm}^{2}$ is loaded into the vacuum chamber and with similar conditions used with the other structures.

Figure 5-1 represents the FE I-V characteristics of tungsten oxide nanowires and the corresponding F-N plots. The I-V curve exhibits high slope at the operating voltage interval and slow saturation behavior starting at $20 \mathrm{Volts} / \mu \mathrm{m}$.

The FE investigations reveal poorer emission than the CNTs with a higher turn-on electric field of $10 \mathrm{Volts} / \mu \mathrm{m}$ and lower maximum current density of $6.66 \mu \mathrm{A} / \mathrm{cm}^{2}$ at 25 Volts $/ \mu \mathrm{m}$. Although the optimization of this kind of structures through controlling the growth parameters and the density is possible, CBNs seems to have better properties and exhibit improved FE than nanowires. In addition, some kind of metallic nanowires can suffer oxidation affecting their lifetime which might limit their applicability.

Other groups have achieved good results while characterizing tungsten oxides nanowires as field emitters; a current density of $1 \mathrm{~mA} / \mathrm{cm}^{2}$ at a threshold electric field of $22 \mathrm{~V} / \mu \mathrm{m}$ and other good properties are reported elsewhere. ${ }^{16,117}$ However, the turn-on and threshold field produced from nanowires are still too high to be considered for many applications such as FEDs. 


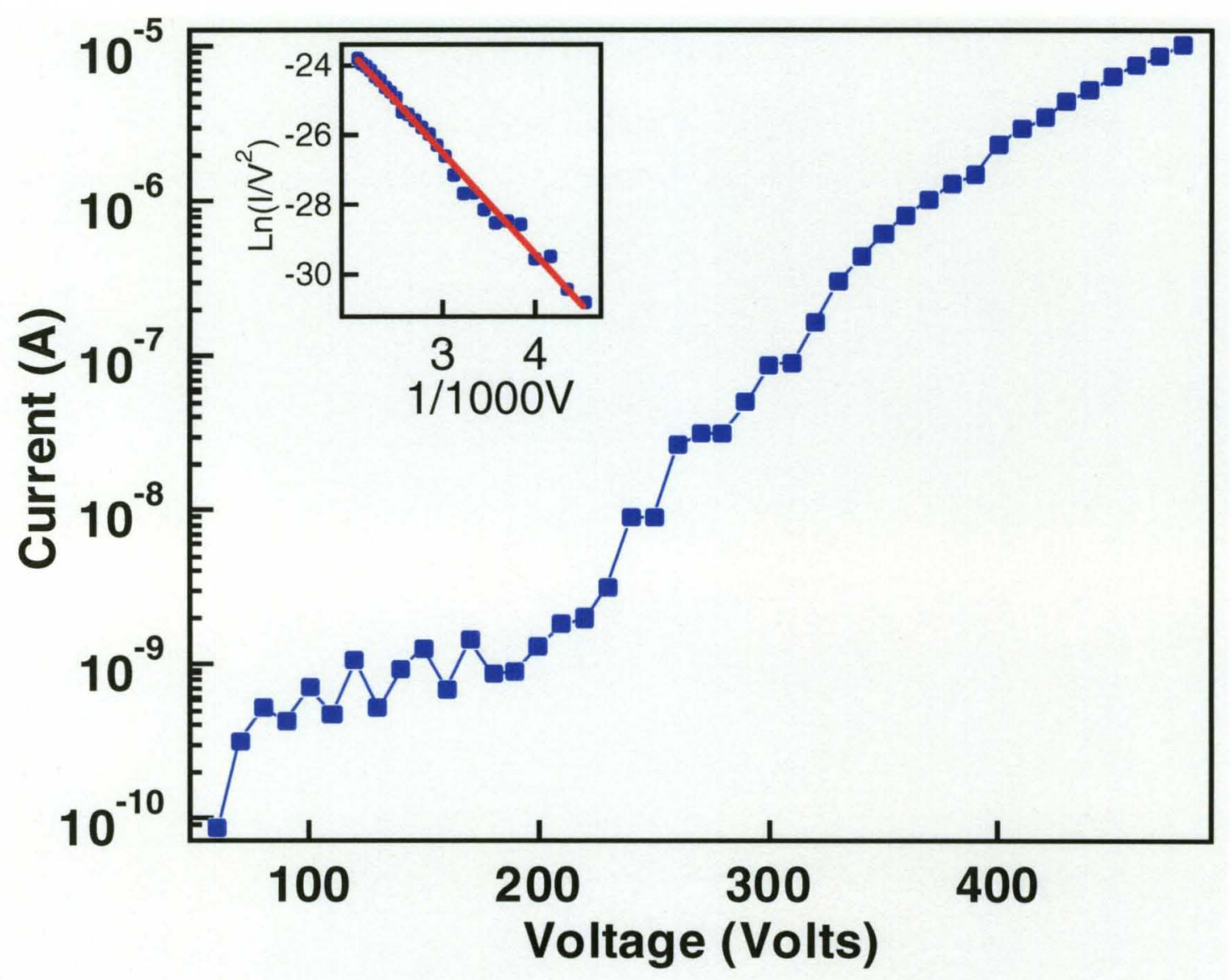

Figure 5-1 FE I-V curves and the inset is the corresponding F-N plot of a tungsten oxide nanowires carpet. 


\section{5-2. Characterization of Semiconducting MWCNTs:}

Field emission from Carbon Nanotubes (CNTs) has been explored for potential applications varying from flat panel displays to miniature scanning electron microscope columns. ${ }^{118}$ CNTs are known to have most of the favorable properties as field emitters such as high aspect ratio, good mechanical, electrical, thermal and chemical properties, and ability to be grown as vertical arrays with controlled density. ${ }^{19,120}$ The dependence of field emission properties of carbon nanotubes on (i) diameter (ii) length (iii) density/sparsity (spacing between neighboring tubes) (iv) alignment/randomness, (v) wall defects, and (vi) surface absorbates has been explored to a greater depth. ${ }^{121}$ Higher values of $\beta$ results in higher local electric field (low turn on fields) as well as high current densities, which is extremely desirable in many applications such as X-ray devices.

Although high $\beta$ values $(2,500-10,000)$ and low turn-on fields $(0.8-1.5 \mathrm{~V} / \mu \mathrm{m})$ have been reported for single walled carbon nanotubes (SWCNTs) and multi walled carbon nanotubes (MWCNTs), very limited work has been dedicated to their exploitation

as hot cathodes. ${ }^{122-124} \mathrm{CNTs}$ encouraging FE properties are expected to be among the candidates for the future electron sources due their high aspect ratio and local field enhancement. Their applicability as efficient electron emitters for applications in electron, X-ray sources and energy conversion devices is also possible.

Figure 5-2 is a SEM image of dense and super tall MWCNTs grown by PECVD. The sample consists of $1 \mathrm{~cm}$ tall nanotubes which are closely packed with a high density of $3 * 10^{9}$ nanotube/ $\mathrm{cm}^{2}$, an average length of $1 \mathrm{~cm}$ and an average diameter of $\sim 100 \mathrm{~nm}$. 


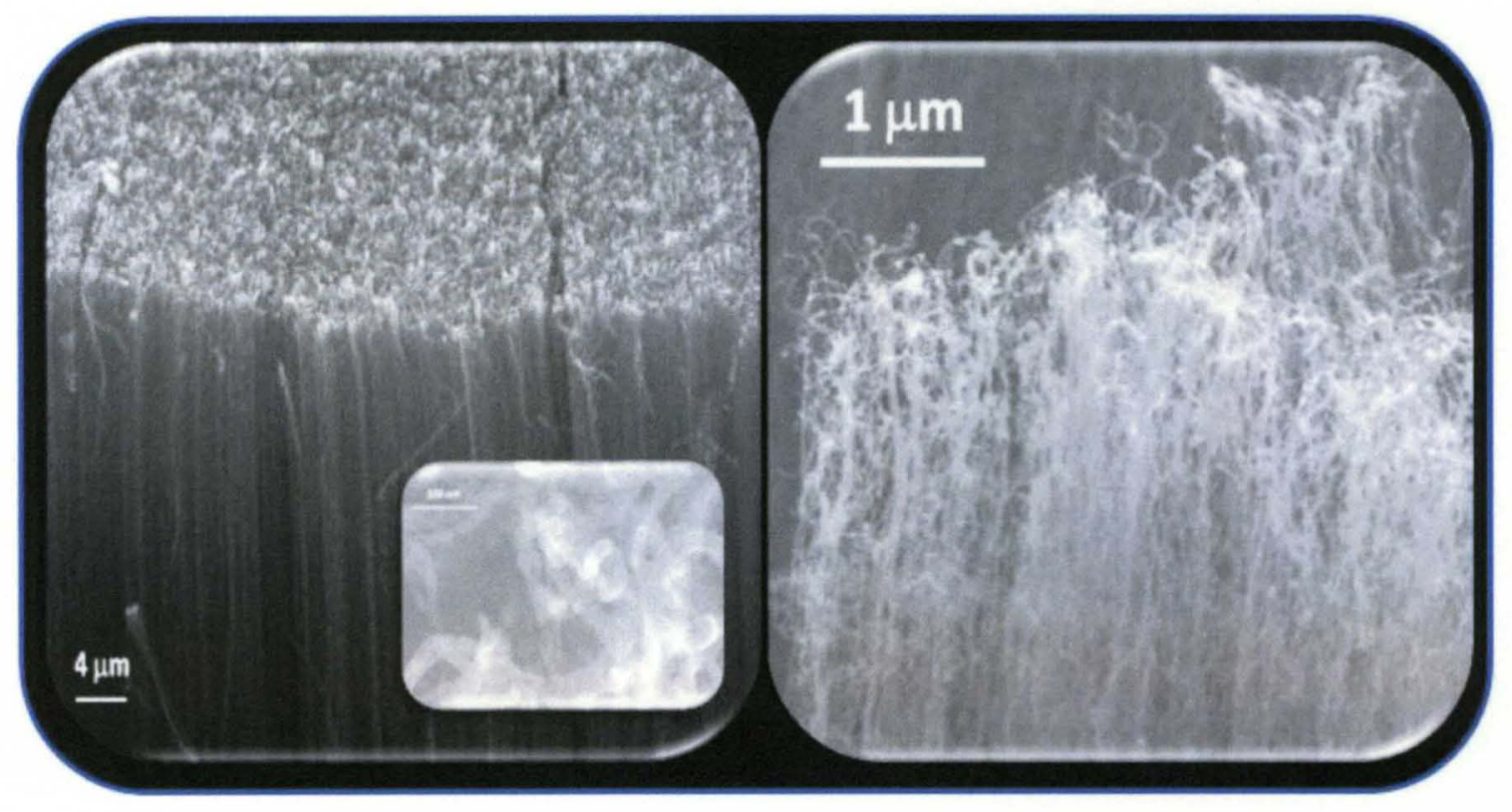

Figure 5-2: (a) SEM images of MWCNTs carpets, (b) Top view. ${ }^{125}$

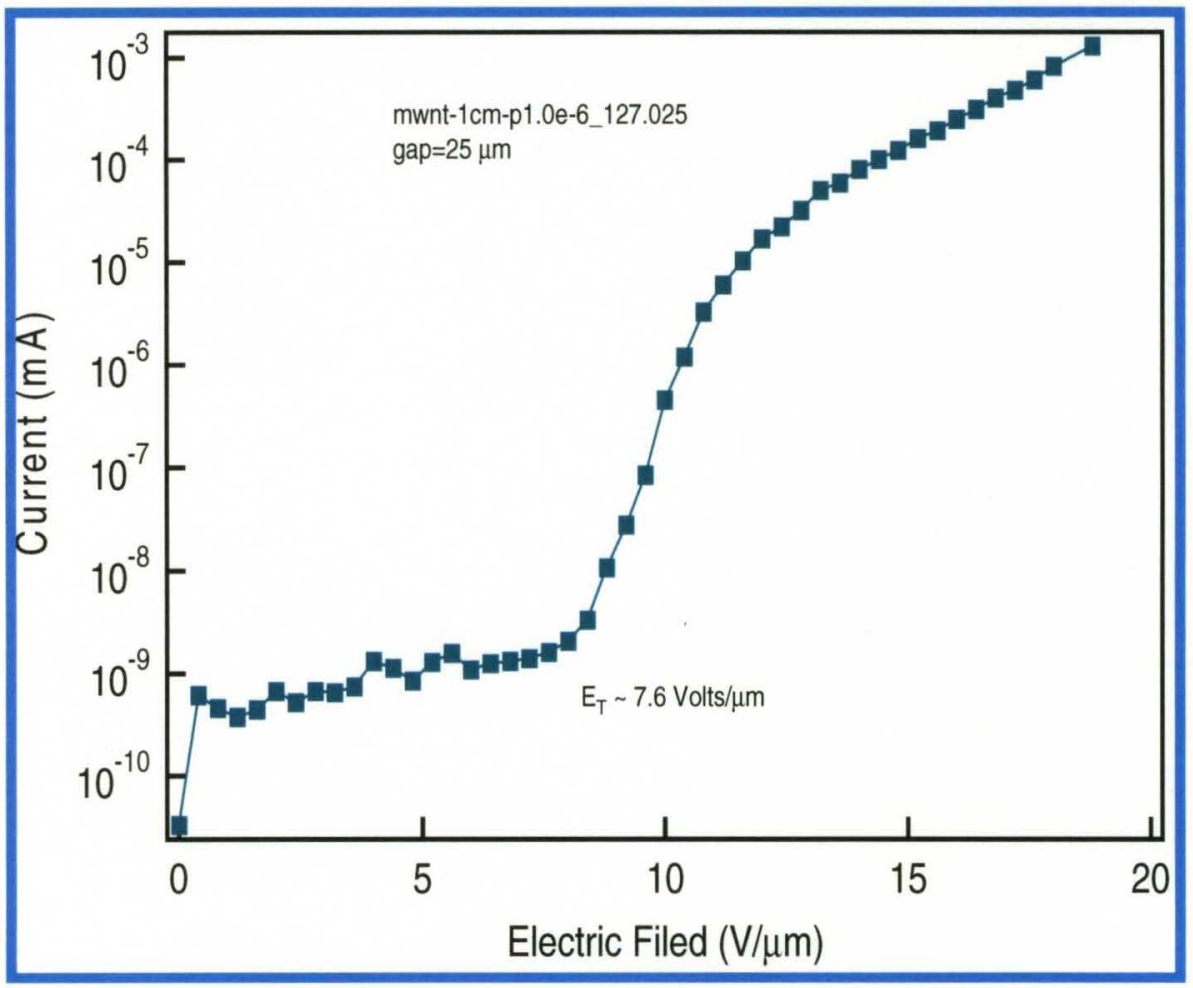

Figure 5-3: I-V curves of a super long carpet of MWCNTs. 
Figure 5-3 illustrates the room temperature FE behavior of MWCNTs at vacuum pressure $\sim 10^{-7}$ Torr. The I-V curve shows three regions having different slopes. The first one is the region of no emission and only the background current is recorded. A sharp change in the slope appears indicating the detection of emission current. The corresponding turn-on electric field is $7.6 \mathrm{~V} / \mu \mathrm{m}$. According to the models initiated by Murphy and Good, and duplicated in the present study for multiple materials including MWCNTs, the field amplification should play a significant role in lowering the turn-on electric field. As it is mentioned in the previous chapters, to a first approximation, the field enhancement factor is proportional to the aspect ratio of the emitter.

When the applied voltage is further increased, a second knee appears and the slope changes rapidly at $12 \mathrm{Volts} / \mu \mathrm{m}$ (within the voltage interval of $300-500 \mathrm{Volts}$ ). During this step, the current increases by two orders of magnitudes at a slower rate and the corresponding F-N plot also reflects the presence of a knee by observing a change in its slope. This region is usually referred to as the saturation.

The values obtained for the turn-on and operating voltages is relatively high and the emission can be considered as poor. The poor properties can be attributed to the existence of high field screening effect observed by the neighboring tubes. Since the tubes are adjacent to each other, one should expect poor emission properties from such sample even with higher threshold fields. Nevertheless, the field enhancement factor obtained using F-N analysis is 1400; moreover, the current extracted from this sample reached few milliamperes. According to the theory and to modeling, no enhancement can be seen from adjacent nanotips except at the edges of the nanotubes carpet. 
To understand the reason behind this inconsistency, image spectroscopy of the samples under investigation is performed. In addition, two samples with similar densities and different heights are characterized for FE behavior (Figure 5-2). According to Figure 5-2, the non uniformity and high density is clearly depicted and many individual and bundles of nanotubes are protruding from the rest of the carpet. Emission from highly dense CNT cathodes can be dominated by edge emission and hot spots due to the concentration of the electrical field at edges and protrusions of the CNT carpet. Therefore, protrusions and edges can easily dominate the emission process leading to poor emission properties.

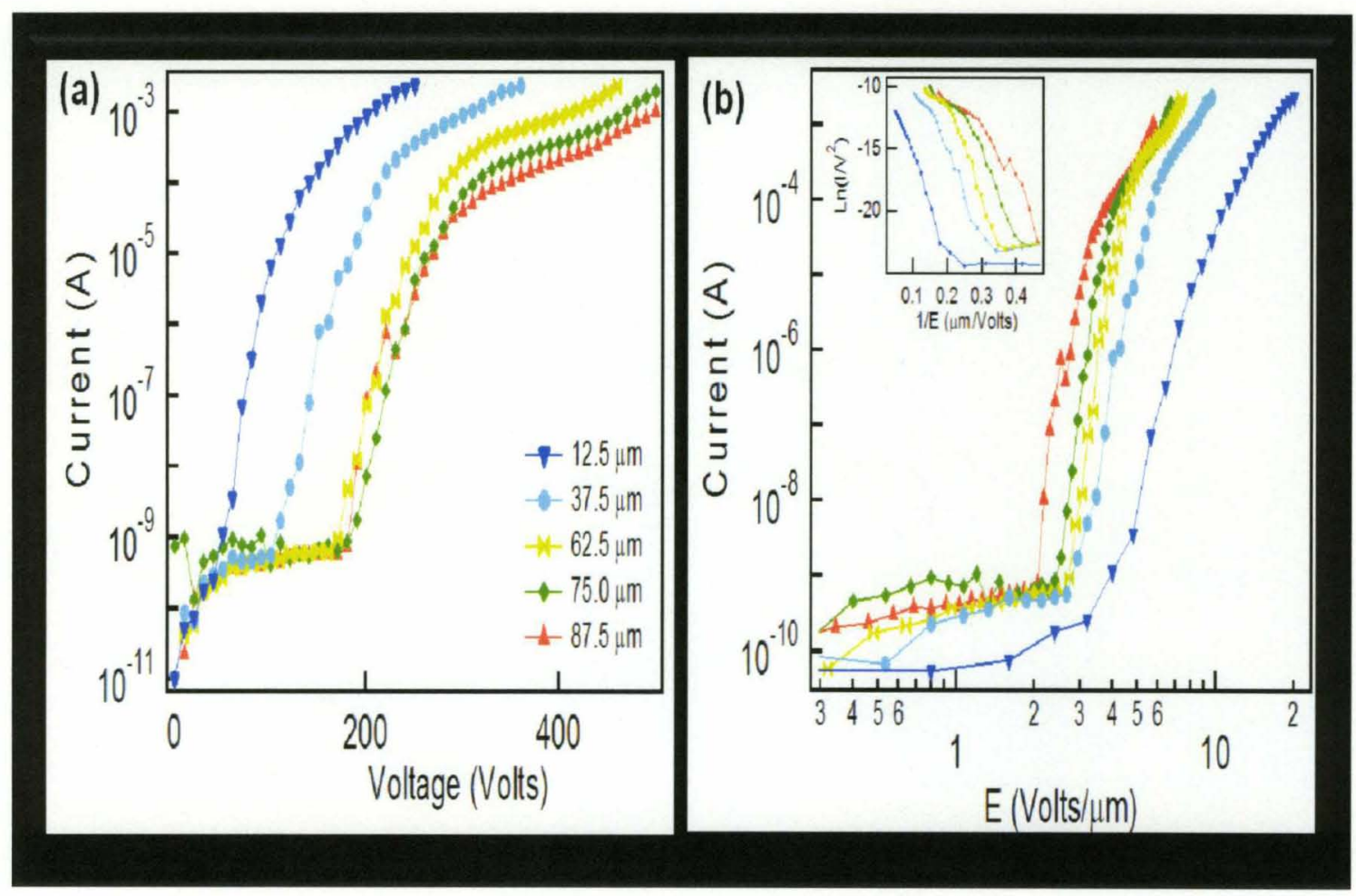

Figure 5-4: (a), (b) FE characteristics and the inset is the corresponding F-N plot of a super long MWCNTs carpet. ${ }^{126}$ 
Figure 5-4 presents the FE behaviors of super long MWCNTs at different separations, in linear and logarithmic scales, and the corresponding F-N plot. It is clear that there is instability in both the I-V and the F-N plots, especially at higher separations (lower fields) by reason of the carpet's no uniformity and high field screening effect.

Figure 5-5(a) displays the I-V characteristics of a shorter MWCNTs sample at various anode-cathode separations. The same behavior is observed where three regions are present for each separation. At higher separations, little instability in the emission current is observed at the emission operating voltage. Even though this sample is shorter than the previous one, it exhibits better FE properties including better stability and higher emission current. Therefore, the aspect ratio advantage can be nullified through the use of highly dense emitters and the field screening affect is not taken into consideration. Figure 5-5(b) shows the FE response of the same sample during 10 hours emitting period. The emission starts to fluctuate at higher rate and the current dropped by $\sim 30 \%$ to show better stability at $0.3 \mathrm{~mA}$.
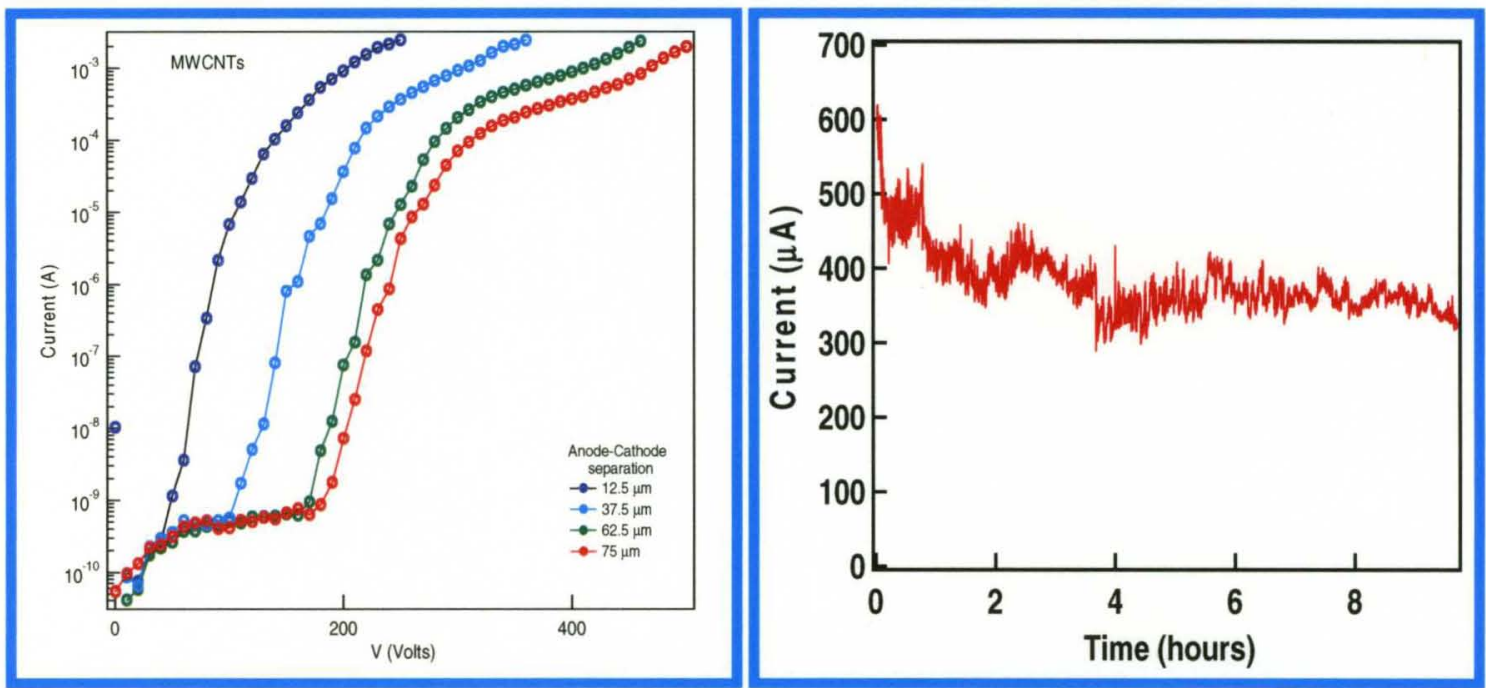

Figure 5-5: (a) I-V curves of a short MWCNTs carpet at different distances and (b) Emission current versus time. ${ }^{127}$ 


\section{5-3. FE characterization of CCNTs}

\section{5-3-1. Bulk characterization}

Several CCNT arrays with different morphological characteristics (tip radius, aspect ratio, density and wall structure) are synthesized using variations in the growth process parameters. The field emission characteristics for a CCNT array sample with a tip radius of $5 \mathrm{~nm}$, moderate inter-emitters distance with a density of $10^{8} / \mathrm{cm}^{2}$ and having the highest aspect ratio exhibited a low turn-on electric field $(<0.7 \mathrm{~V} / \mu \mathrm{m})$ and a high field enhancement factor $(\beta>7,500)$. The reduced emission characteristics from other samples are attributed either to the presence of field screening effect resulting from higher CCNTs density and due to the corresponding tip and wall structures.

Long CCNTs grown vertically with different densities, together with their tapered morphologies can be well separated at their tips..$^{91,128,129}$ Tapered emitters are expected to be mechanically more stable than a constant diameter nanotube of the same tip diameter. Also, tapered carbon structures, due to their increasing cross sectional area away from the tip, increase the thermal transport and are likely to sustain greater current densities than carbon nanotubes. Field emission measurements on CCNTs grown with metal catalysts have been reported. ${ }^{130,131}$ However, the extent of the above studies is limited and the structures studied do not represent the ideal conical morphology due to the presence of metal contamination at their tip. In the present work, several CCNT array samples were synthesized by adjusting growth parameters that control aspect ratio, density and wall structures in order to find the optimum condition for better electron emitters. The results show that the performance of the CCNT arrays is dependent upon their morphology and can match or exceed that reported for SWCNTs and MWCNTs ${ }^{122-124}$. 
In order to verify the origin behind the poor emission from dense and super long CNTs, as well as optimize the design of an efficient FE based device, further investigations are carried out where samples having well defined densities, aspect ratios and morphologies are employed. Five different samples of CCNTs arrays are grown on $500 \mu \mathrm{m}$ diameter platinum wire. FE measurements are performed on each sample within vacuum chamber at a pressure of $10^{-7}$ Torr. Measurements are performed on the wire with and without CCNT at 5 different distances $(d)$ by sweeping the voltage from 0-500 Volts while recording the change in current using the Keithley 6487 Pico-ammeter.

The variation in time scales used for both the process steps during the synthesis of CCNTs resulted in variation in the density, length and the morphology of the CCNT arrays. The experimental conditions employed and the resulting FE characteristics of CCNTs for sample\#1-3 were summarized in Table 5-1. Increase in the time of step 1 increases the density and length, with $\sim 80 \%$ of the CCNTs within a few $100 \mathrm{~nm}$ of their average length. Increasing the duration of step 2 increases the etching of the CCNTs and also reduces the density of CCNTs. Figure 5-6 shows the SEM images of these three samples of CCNT array grown on a platinum wire. The corresponding insets present the enlarged view of individual CCNT tips, which show the absence of any metal catalyst at the tip. Measurement on a Pt wire coated with just amorphous carbon and no CCNTs, results in no detectable emission current. Therefore, the detected current is due to FE from CCNTs. 
Table 5-1: Experimental conditions, the resulting structural characteristics, and the field emission properties of the three CCNT array samples used in this study.

\begin{tabular}{|c|c|c|c|c|c|c|c|c|c|}
\hline \multirow{3}{*}{$\begin{array}{c}\text { Sample } \\
\#\end{array}$} & \multicolumn{3}{|c|}{$\begin{array}{l}\text { Experimental } \\
\text { conditions }\end{array}$} & \multicolumn{3}{|c|}{$\begin{array}{l}\text { Structural } \\
\text { properties }\end{array}$} & \multicolumn{3}{|c|}{$\begin{array}{c}\text { Field emission } \\
\text { properties }\end{array}$} \\
\hline & \multicolumn{2}{|c|}{$\begin{array}{l}\text { Process time } \\
\quad(\mathrm{min})\end{array}$} & \multirow{2}{*}{$\begin{array}{l}\text { Power } \\
\text { (W) }\end{array}$} & \multirow{2}{*}{$\begin{array}{l}\text { Density } \\
\left(10^{8} / \mathrm{cm}^{2}\right)\end{array}$} & \multirow{2}{*}{$\begin{array}{c}\text { Average } \\
\text { length } \\
(l) \\
(\mu \mathrm{m})\end{array}$} & \multirow{2}{*}{$\begin{array}{l}\text { Average } \\
\text { radius } \\
(r) \\
(\mathrm{nm})\end{array}$} & \multirow{2}{*}{$\beta_{\max }{ }^{*}$} & \multirow{2}{*}{$\begin{array}{c}E_{T}^{\left.*{ }^{*}\right)} \\
(\mathrm{V} / \mu \mathrm{m})\end{array}$} & \multirow{2}{*}{$\begin{array}{l}I_{\max }{ }^{* c)} \\
(\mu \mathrm{A})\end{array}$} \\
\hline & Step 1 & Step 2 & & & & & & & \\
\hline 1 & 120 & 150 & 900 & 2.2 & $9.0 \pm 0.1$ & $5 \pm 1$ & 7600 & 0.66 & 520 \\
\hline 2 & 30 & 165 & 980 & 4.5 & $7.0 \pm 0.1$ & $13 \pm 1$ & 2313 & 1.5 & 320 \\
\hline 3 & 15 & 165 & 980 & 0.7 & $4.0 \pm 0.1$ & $12 \pm 1$ & 1324 & 2.3 & 200 \\
\hline
\end{tabular}

*a) Maximum field enhancement factor measured.

*b) Lowest measured turn on electric field.

${ }^{* \mathrm{c})}$ Maximum current corresponding to threshold electric field of $3.6 \mathrm{~V} / \mu \mathrm{m}$

Table 5-1 illustrates the experimental conditions, the resulting structural and the field emission properties of the three CNP array samples used in this study. Experimental conditions consist of the growth process time and the microwave power employed for the synthesis of all five CCNT samples. Structural characteristics include the average length, estimated density, and the average radius of curvature at the tip of each CCNT sample from SEM image analysis. The results section includes the maximum $\beta$, the lowest $E_{T}$, the maximum emission current per single CCNT. 


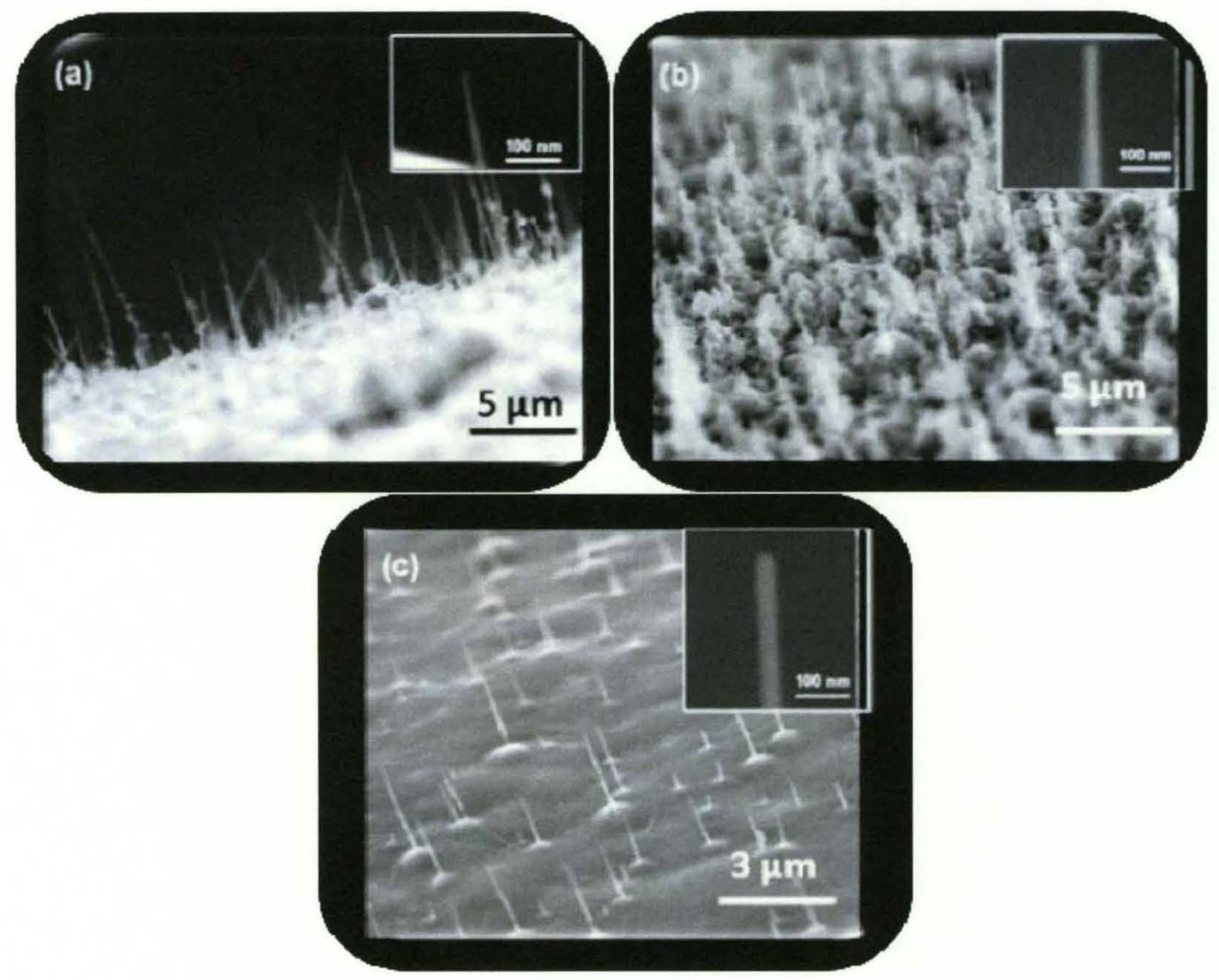

Figure 5- 6: SEM images of three samples of CCNTs with insets showing the enlarged view of the corresponding CCNT tip of (a) sample\#1 (b) sample\#2 and (c) sample\#3. ${ }^{108}$

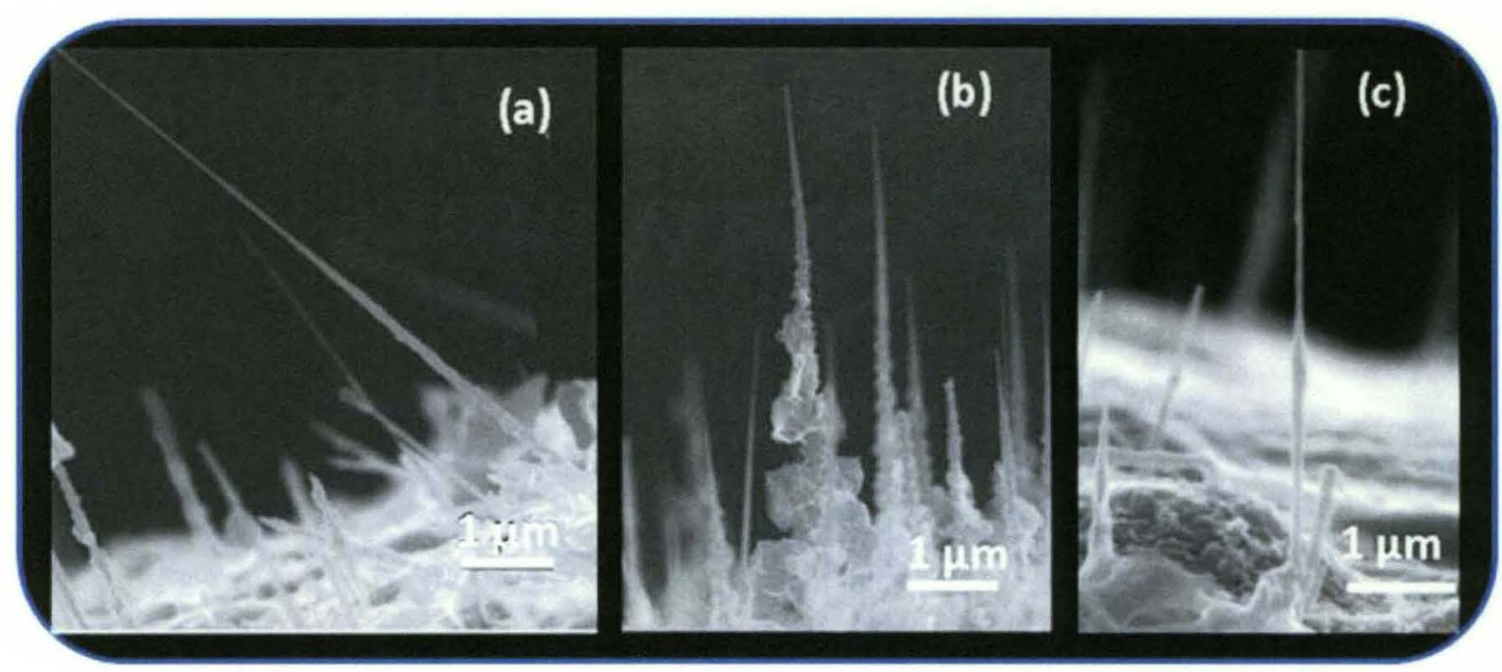

Figure 5- 7: SEM images of the individual CCNT illustrating the variation in the wall structure and aspect ratio, (a) sample\#1 (b) sample\#2 and (c) sample\#3. ${ }^{108}$ 
Figure 5-7 shows the SEM images of the individual CCNT of these three samples clearly depicting the variations in the wall structure and morphology. Sample \#1 with the longest carbon deposition step, has CCNTs with the highest aspect ratio (Figure 5-7(a)), moderate density and also the smallest tip radius estimated from SEM image spectroscopy (Figure 5-7) indicated in Table 5-1. Sample \#2 has CCNTs with lower aspect ratio and larger tip diameter than sample\#1, in addition to the presence of carbon flakes along the length of each CCNT as depicted in Figure 5-7(b). Sample \#3 with longer etching step and a shorter deposition time resulted in CCNTs with the lowest aspect ratio and uneven etching as clearly seen in Figure 5-7(c). CCNTs of sample\#1 with small tip radius, optimum density and high aspect ratio accounts for the enhanced field emission characteristics as described in the following analysis.

The emission current $(I)$ is measured as a function of the applied electric field $(E=V / d)$ for sample\# 1 at each of the five inter-electrode distances $(d)$ as shown in Figure 5-8(a). According to the F-N equation, the electric field $\left(E_{\text {eff }}\right)$ at the tip of CCNT produces an emission current density $(J)$ :

$$
J=\frac{A\left(E_{e f f}\right)^{2}}{\Phi} e^{-\left(\frac{B \Phi^{3 / 2}}{E_{\text {tff }}}\right)}
$$

Where $E_{\text {eff }}$ denotes the local electric field present at the tip of CCNT.

Figure 5-8(a) shows the I-V characteristics produced by sample\#1 that consist of two knees; the first one appears where a sudden change in that the emission current is detected. Then the current begins to saturate at a second knee for sample\#1. This observed saturation may be associated with heat induced changes at the tip. ${ }^{132}$ 
(a)

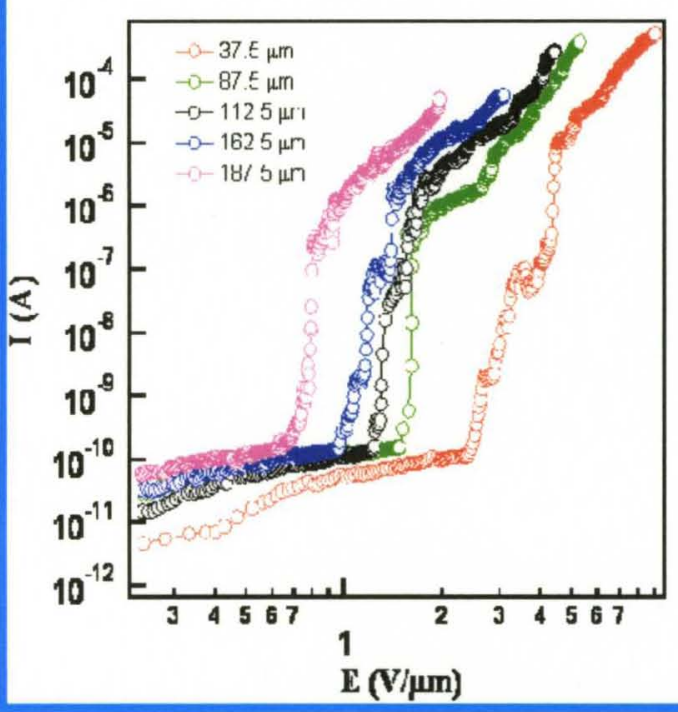

(b)



Figure 5-8: (a) I-V curves and (b) the corresponding F-N plot of CCNTs. ${ }^{108}$

The maximum electric field reported for multiwall carbon nanotubes is $\sim 8 \mathrm{~V} / \mathrm{nm}$ before undergoing tip failure (due to deformation, evaporation, thermal runaway or arcing). ${ }^{133}$ The estimation of the current density in CCNTs samples is complicated by the curved platinum support. However, effective electric fields $\left(\beta E_{a p p}\right)$ of $\sim 8 \mathrm{~V} / \mathrm{hm}$ and current densities greater than $3 \mathrm{~A} / \mathrm{cm}^{2}$ are reached at the second knee for sample\#1 at a distance of $187.5 \mu \mathrm{m}$, without experiencing thermal runaway or tip failure.

Figure 5-8(b) shows the corresponding F-N plots of $\ln \left(J / E^{2}\right)$ vs. $1 / E$ at each $d$ value. This plot represents the emission current region between the two knees of Figure 5-8(a). The linearity of the plot is indicative of the field emission in the operating current regimes. The slope of this linear plot is given by $\left(\frac{B \Phi^{3 / 2}}{\beta}\right)$. The value for the work function used to compute the values for $\Phi$ is taken to be $4.8 \mathrm{eV}$ based on the TE measurement on the CCNTs. 


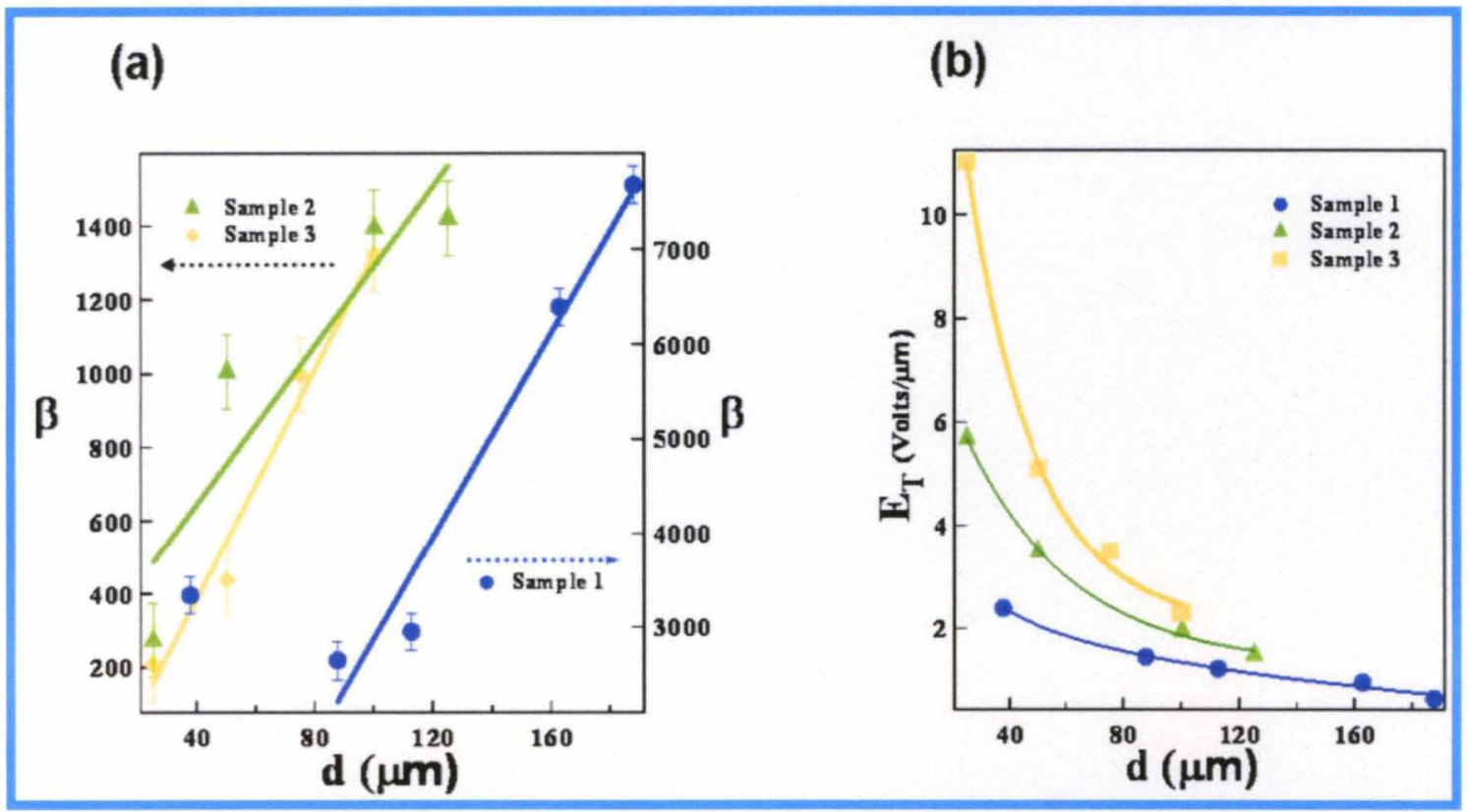

Figure 5-9: Plots of (a) field enhancement factor $\beta$ and (b) turn-on electric field as a function of distance for samples 1-3.

Among the three samples, sample\#1 has the highest value of $\beta(7,600)$ as shown in Figure 5-9(a) which enables the CCNT array to continue to emit up to $d=187.5 \mu \mathrm{m}$ (for the maximum source voltage available) Figure 5-9(b). This high value of $\beta$ is due to a combination of factors such as small radius of curvature at the tip, high aspect ratio, moderate emitter density of CCNTs in sample\#1 and the increased distance $(d)$. In the previous study on CCNTs terminated with nickel catalyst particles, the field enhancement factor as low as 80 have been reported. ${ }^{12}$ The value of $\beta$ for samples 2 and 3 ranges from $2,000-3,000$. Sample 3 has the poorest emission properties of the three samples due to the formation of amorphous carbon along the side walls of CCNT resulting from the prolonged etching which minimizes the edge plane emission sites. ${ }^{134}$ Figure 5-9(a) shows a linear dependence of experimentally derived $\beta$ on the distance $d$, plotted for each of the three samples. The value of $\beta$ determined by FE study is large when compared to the geometrical enhancement factor given by $h / r$, where $h$ is the length of the CCNT and $r$ is 
the radius of curvature at the tip. This deviation was also observed earlier for highly dense carbon nanotube arrays which demonstrated that $\beta$ depends on the inter-electrode distance $(d)$ and other factors such as tip radius, aspect ratio ${ }^{135}$ and field screening effect. $^{14}$ In the present case this discrepancy can be attributed to the structural characteristics of these conical morphologies with open edges on the outer surface acting as emission sites. The effects of the surface adsorbates also cannot be ruled out. ${ }^{136}$ Figure 5-9(b) shows that turn-on electric field ( $E_{\mathrm{T}}$, electric field corresponding to emission current of $1 \mathrm{nA}$ ) decreases with increasing distance $(d)$ for all three samples, reaching a value as low as $0.7 \mathrm{~V} / \mu \mathrm{m}$ (Figure 5-9(a)) for sample\#1 at the maximum possible separation distance, which can be accounted for the high $\beta$ value. This low turn-on electric field, value is comparable to the best values reported for SWNTs and MWNTs. $^{122-124}$

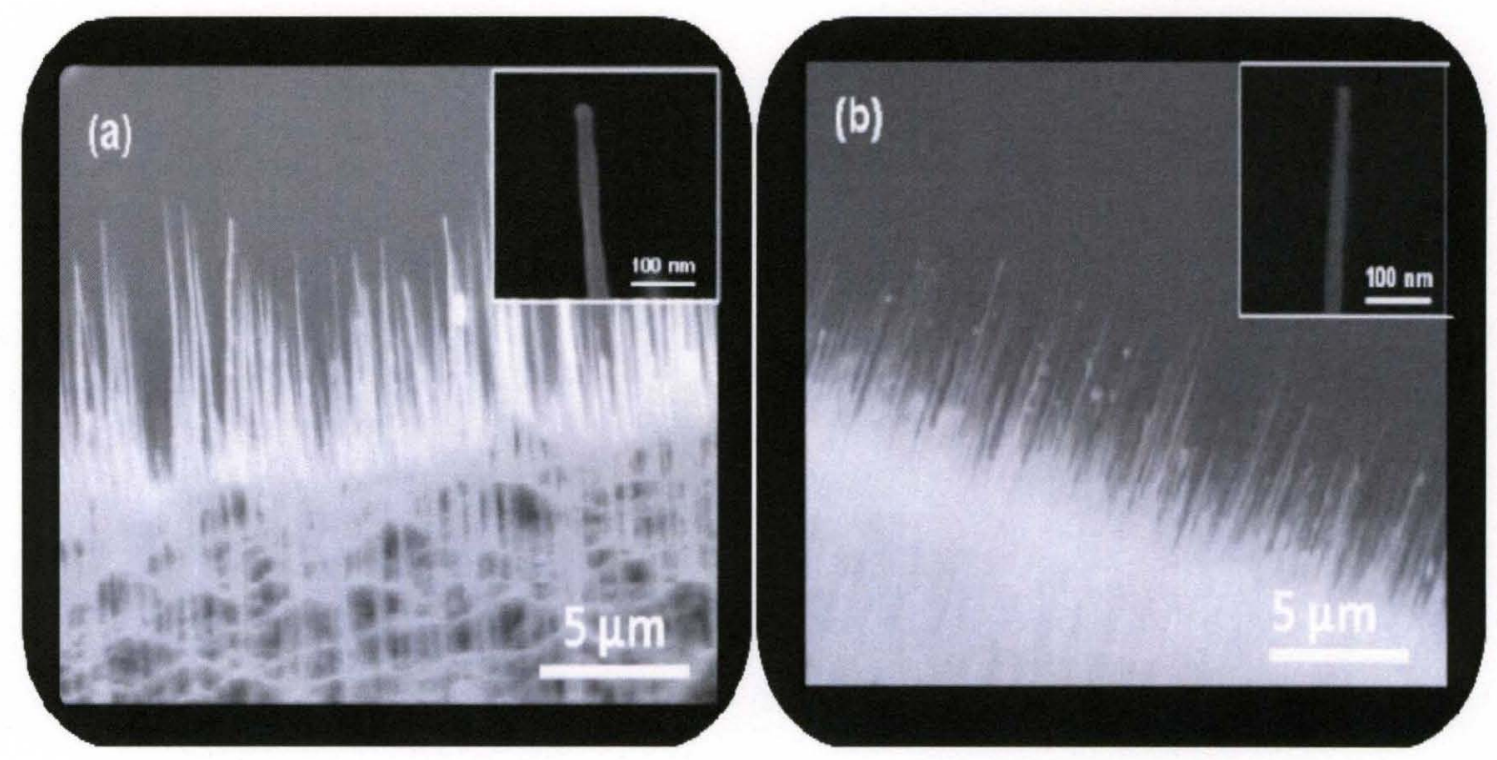

Figure 5-10: SEM images of CCNT arrays of (a) sample 4 (b) sample 5, with insets showing the enlarged view of the corresponding CCNT tip. ${ }^{108}$ 
Among the five samples, samples 4 and 5 are varied in the CCNTs density while other structural characteristics such as radius of curvature at the tip, length, and wall structure are maintained the same. Figure 5-10 shows the SEM images of (a) sample 4 and (b) sample 5 where the increased density of sample 5 is clearly seen. Figure 5-11 shows that sample 4 has better emission properties compared to sample 5 , which can be attributed to the reduced field screening affect provoked by the proximity of neighboring emitters due to lower density. In fact, the inter-emitter distance should be at least 1-2 times the emitter length to reduce significantly the field screening effect. ${ }^{14}$ The interemitter distance of sample 4 is about half of the emitter's average length. Therefore, according the simulation and references, the emitters still carry on some screening to each other. $^{14}$

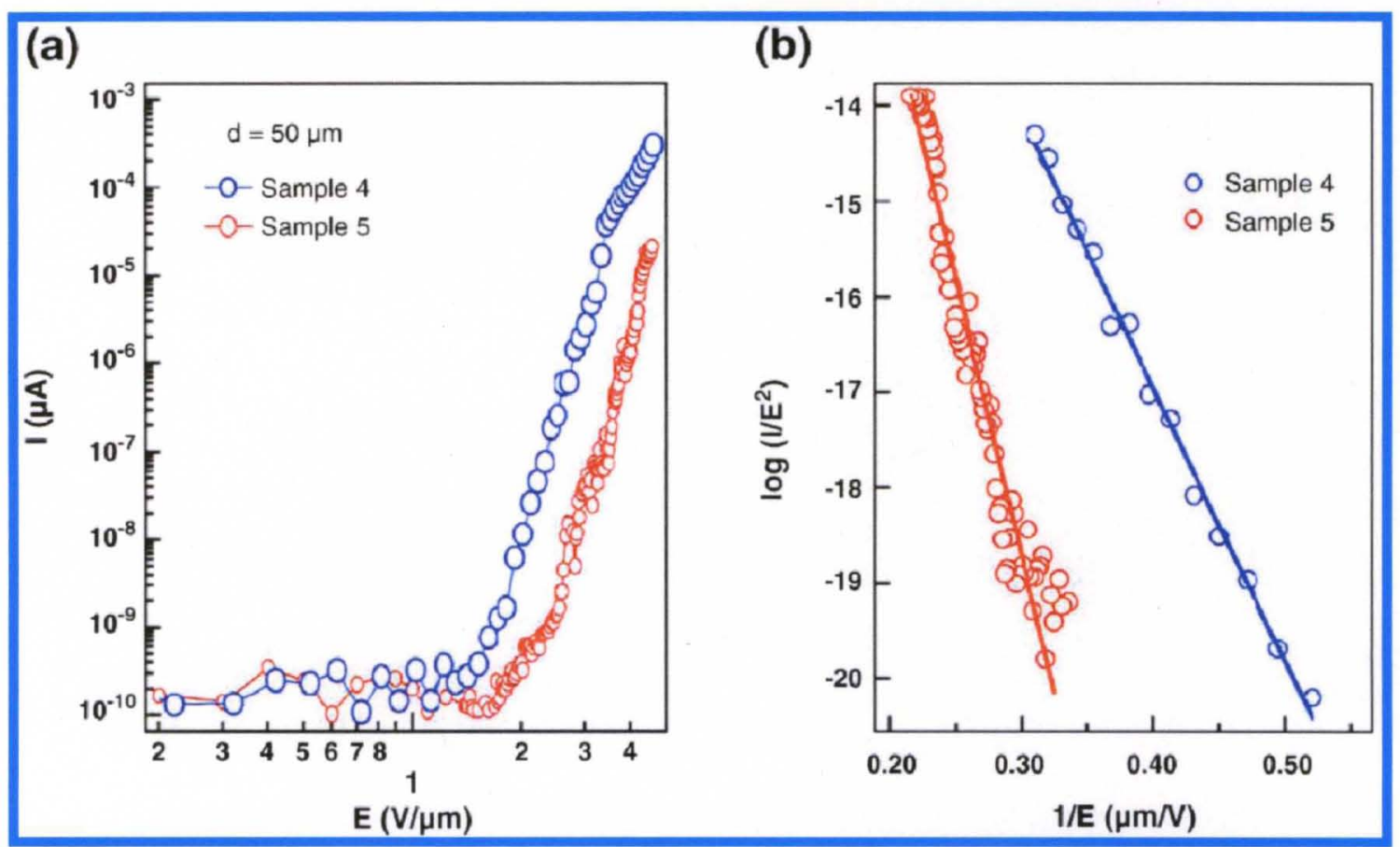

Figure 5-11: The I-V characteristics of sample 4 and 5 (a) Current vs. the macroscopic electric field (b) corresponding F-N plots. ${ }^{108}$ 
The structural characteristics, deduced from SEM imaging spectroscopy and emission characteristics of sample 4 and 5 are shown in Table 5-2. With comparable radii of curvatures, length, and while the emitters densities are different, the obtained value for $\beta$ of sample\#1 is more than the double of the one of sample\#5. In addition, the current density at $3.6 \mathrm{~V} / \mu \mathrm{m}$ is 15 times larger in the case of sample\#4.

Table 5-2: Shows a comparison of the resulting structural characteristics and the field emission properties of the two CCNTs array samples that are morphologically similar with different densities

\begin{tabular}{|c|c|c|c|c|c|c|}
\hline \multicolumn{3}{|c|}{$\begin{array}{c}\text { Structural } \\
\text { properties }\end{array}$} & \multicolumn{7}{c|}{$\begin{array}{c}\text { Field emission } \\
\text { properties }\end{array}$} \\
\hline Sample \# & $\begin{array}{c}\text { Density } \\
\left(10^{8} / \mathrm{cm}^{2}\right)\end{array}$ & $\begin{array}{c}\text { Average } \\
\text { length }(1) \\
(\mu \mathrm{m})\end{array}$ & $\begin{array}{c}\text { Average } \\
\text { radius }(\mathrm{r}) \\
(\mathrm{nm})\end{array}$ & $\beta_{\max }^{(a)}$ & $\begin{array}{c}E_{T}^{(b)} \\
(\mathrm{V} / \mu \mathrm{m})\end{array}$ & $\begin{array}{c}I_{\max }^{(c)} \\
(\mu \mathrm{A})\end{array}$ \\
\hline $\mathbf{5}$ & 15 & $7.0 \pm 0.1$ & $12 \pm 1$ & 2920 & 1.72 & 307 \\
\hline
\end{tabular}
${ }^{\text {(a) }}$ Maximum field enhancement factor measured.
(b) Lowest measured turn on electric field.
(c) Maximum current corresponding to threshold electric field of $3.6 \mathrm{~V} / \mu \mathrm{m}$ 


\section{5-3-2. In-situ characterization of CCNTs}

In situ FE characterization of individual CCNT provides useful information on the parameters affecting the performance of emitters, on the background and emission optimization. The morphology and shape of the emitter have direct influence on FE properties of cathodes. In situ FE standardized technique is established and proven to be an additional tool for characterization and understanding the FE behavior of a single nanostructure through the determination of multiple properties. This study reveals the capabilities of single CCNT to emit electrons such as the maximum current density per tip, the turn-on filed of individual emitter and the packing density's upper limit for a better and more efficient field emission device. In order to achieve the aforementioned properties, a sharp STM tip is fabricated to approach a single CCNT in a diode configuration set up, as the SEM picture of Figure 5-12(a) presents.
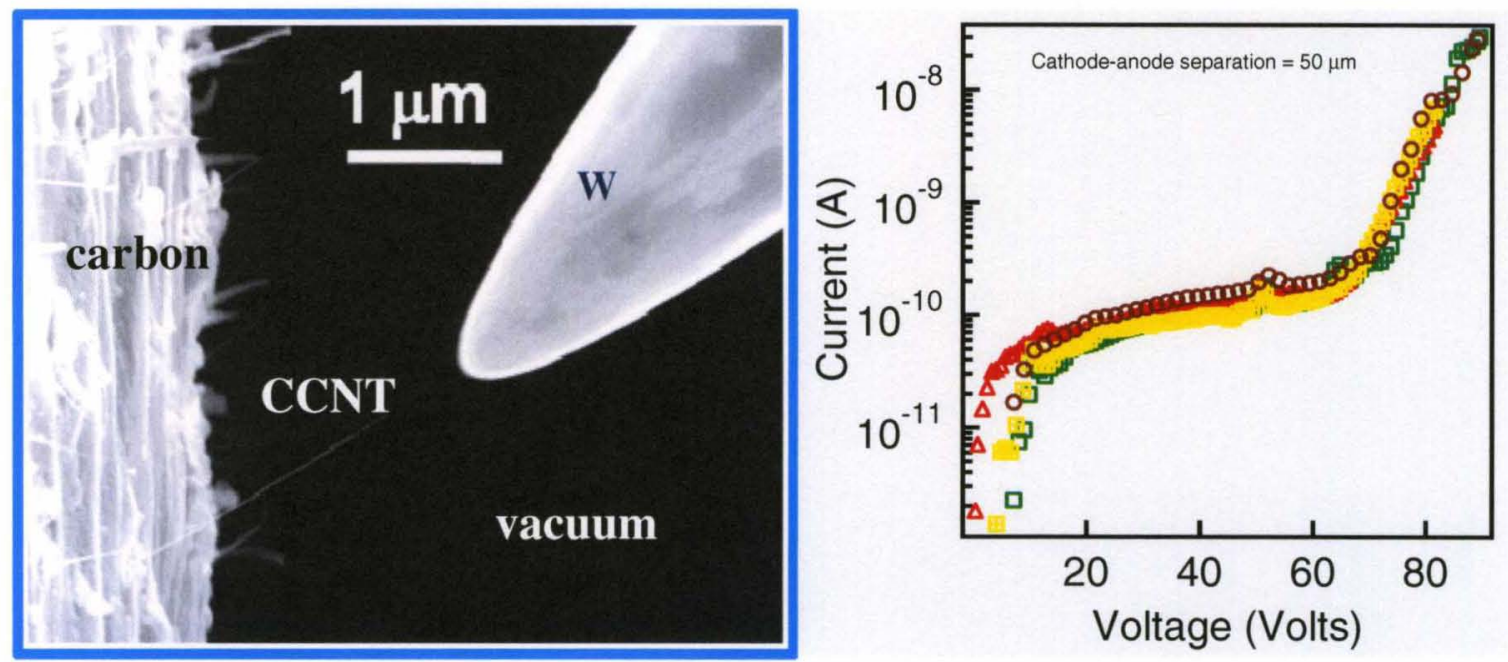

Figure 5- 12: (a) SEM image of a single CCNT in a FE diode configuration next to STM tip (b) I-V characteristics of an individual CCNT at $50 \mu \mathrm{m}$ separation. ${ }^{137}$ 
FE characterization of single CCNT is carried out within the SEM chamber at a vacuum of $2 * 10^{-6}$ Torr, FE is then performed at a separation of $d=50 \mu \mathrm{m}$. An electrochemically etched STM probe, acting as the counter electrode, approaches the tallest single CCNT, usually located in the least dense area of the sample. The voltage is swept from 0 to 100 Volts at $50 \mu \mathrm{m}$ separations and resulted in $0.1 \mu \mathrm{A}$ emission current. Figure 5-13(b) shows multiple curves of the emission current versus the applied field of a single CCNT emitter. These curves confirm the reproducibility and continuity of emission from the same structure. In addition, to eliminate and purify the emitting surface from any preexisting contaminants and achieve an improved emission current, the abovementioned steps can also be adopted to flush the emitting area. According to the curves, there is little instability in the emission current and the I-V characteristics are not perfectly identical, which might be caused by presence of contaminants, due to the rough vacuum conditions, or other emitting sites such as the edges of the graphitic sheet making up the tube and carbon flakes.

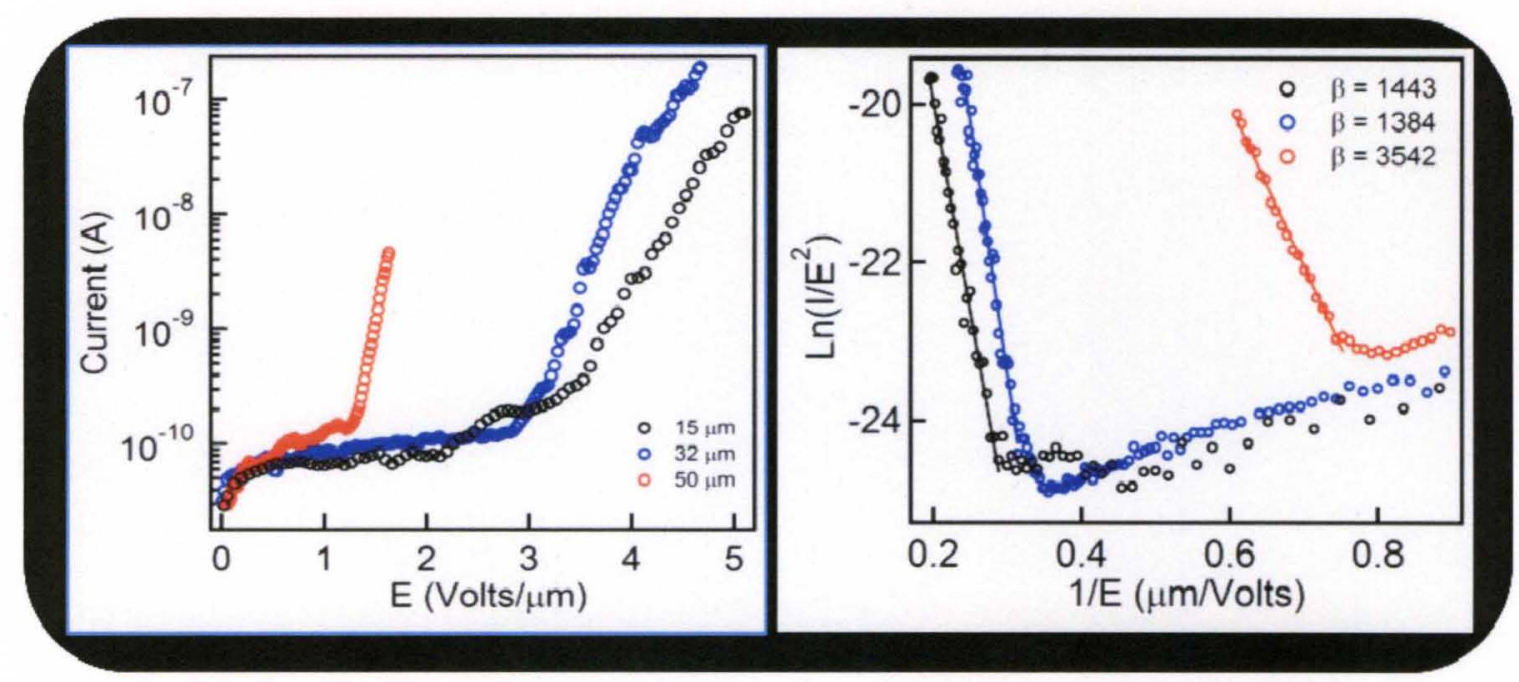

Figure 5-13: (a) I-V curves and (b) the corresponding F-N plot of an individual $\mathrm{CCNT}^{138}$ 
Figure 5-13(a) shows the I-E curves representing the emission current versus the applied electric field for three different CCNT samples that have comparable lengths and radii of curvature. Figure 5-13 (b) is the corresponding F-N plot for each CCNT; it displays a high linearity of the $\operatorname{Ln}\left(I / E^{2}\right)$ versus $1 / E$.

Table 5- 3. Experimental conditions, the resulting structural characteristics, and the field emission properties of the three individual CCNTs.

\begin{tabular}{|c|c|c|c|c|c|c|c|c|}
\hline Sample & $\begin{array}{c}h \\
(\mu \mathrm{m})\end{array}$ & $\begin{array}{c}d \\
(\mu \mathrm{m})\end{array}$ & $\begin{array}{c}E_{T} \\
(\mathrm{~V} / \mu \\
\mathrm{m}) \\
\end{array}$ & $\beta$ & $\begin{array}{l}\boldsymbol{r}_{\text {meas }} \\
(\mathbf{n m})\end{array}$ & $\begin{array}{c}\boldsymbol{r}_{\text {exp }} \\
(\mathbf{n m})\end{array}$ & $\begin{array}{c}I_{\max }(\mathbf{n A}) \\
@ \\
E(\mathbf{V} / \mu \mathrm{m})\end{array}$ & $\begin{array}{c}I_{\max } / \mathbf{c n p} \\
(\mathbf{n A})\end{array}$ \\
\hline 1 & 6 & 15 & 3.3 & 1443 & 5 & 4.55 & $\begin{array}{c}74 \\
\text { (a) } 5.1\end{array}$ & 570 \\
\hline 2 & 6 & 32 & 2.8 & 1384 & 10 & 6.95 & $\begin{array}{c}186 \\
\text { (a) } 4.67\end{array}$ & 610 \\
\hline 3 & 6 & 50 & 1.23 & 3542 & 6.5 & 4.0 & (a) 1.63 & 51 \\
\hline
\end{tabular}

Table 5-3 summarizes the results of FE investigations of three individual CCNTs, measured radius of curvature using image spectroscopy, and the results of the data analysis. The change of radius is due to the change in the etching time during the growth process. Sample \#1 and sample \#3 have comparable radii.

For instance, the critical current that a single CCNT sustains before thermal runway or emitter's failure is an important parameter for evaluating the cathodes' limits. The value for the critical current that an individual CCNT supports is $\sim 2.9 \mu \mathrm{A} / \mathrm{CCNT}$ 
corresponding to a current density of $\sim 1 \mathrm{GA} / \mathrm{m}^{2}$. This value is much less than the current density of $10^{4} \mathrm{GA} / \mathrm{m}^{2}$ achieved by Wang et al. at anode-cathode separation of $300 \mathrm{~nm} .^{132}$

Second, the lower the radius of curvature the lower is the turn-on field. There is a direct dependence of the turn-on field on emitter geometry. Figure 5-14 illustrates change in I-E curve's behavior of an individual CCNT characterized at $100 \mu \mathrm{m}$ separation. Two knees are visible in the graph indicating response of the emitter to the applied voltage. The first one is the turn-on field while the second one indicates changes to the emitter's geometry or morphology. It is similar to a saturation regime with fluctuation in the emission current.

Third, by means of field screening effect and the maximum current that can be extracted from a single emitter, one can determine the packing density and the maximum expected current density achievable using nanostructure array emitters.

Figure 5-14 shows the electrical response of an individual CCNT when the electric field is applied and high emission current is extracted through FE process. As the emission current response is very sensitive to the shape and surface of the emitting area, a change in I-E characteristics is an indicative of the change in tip's shape and/or diameter. This tip induced changes are seen on the emission current where the saturation regime starts. Therefore, the emitter's has a direct influence on the FE properties. Sharper tip produces low turn-on field and low threshold field due to their higher field enhancement factor of $(\beta=3542)$. However, relatively larger diameter pipette can produce higher current densities. 


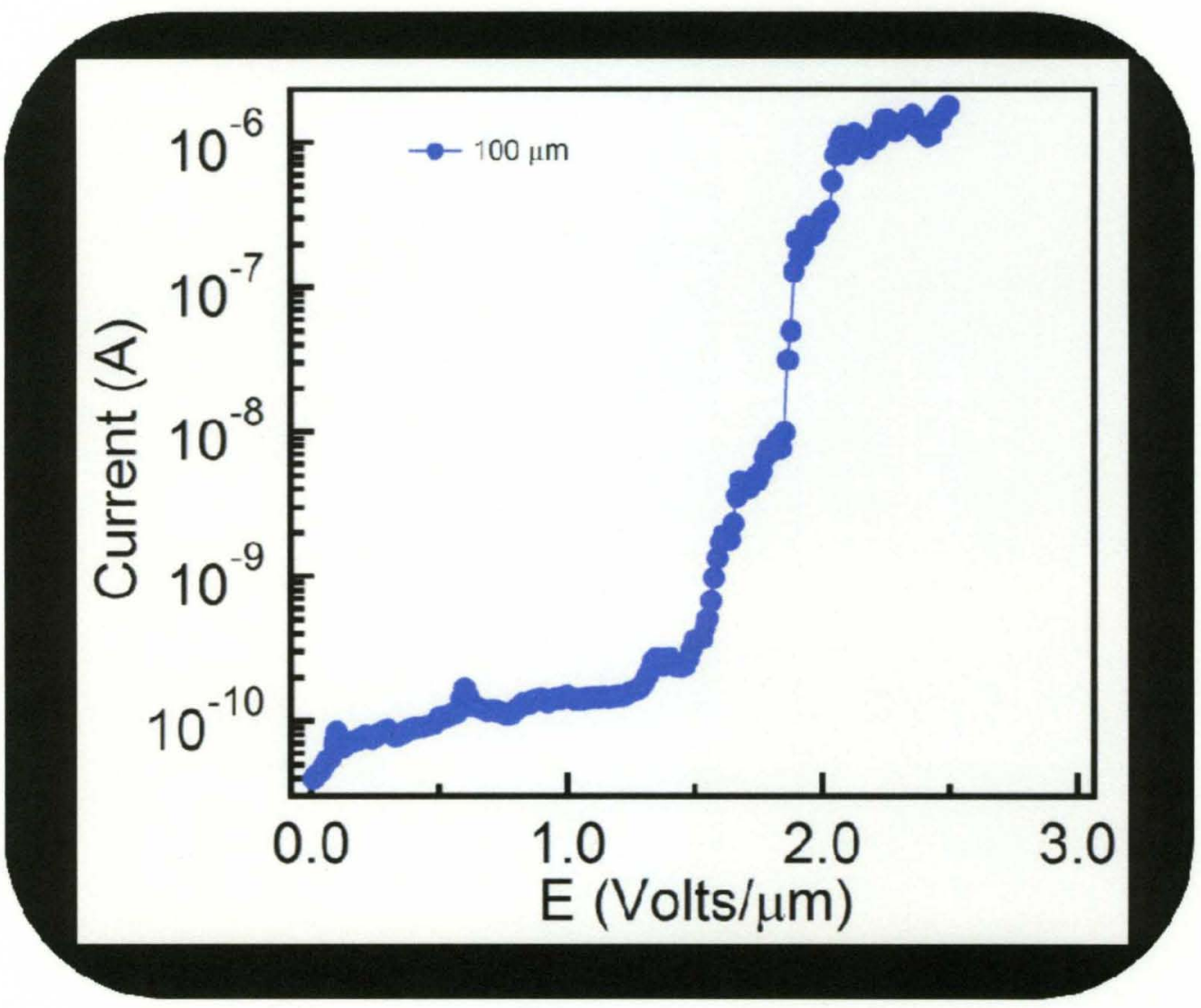

Figure 5- 14: I-E characteristics of an individual CCNT at $100 \mu \mathrm{m}$ separation. 


\section{5-4. FE Characterization of M_SWCNTs}

The M_SWCNTs samples investigated consist of a thin film made of randomly lying single walled carbon nanotubes on a substrate and are $90 \%$ metallic. Unlike the previously introduced nanostructures which are one dimensional structures that are freely standing in a three dimension space, M_SWCNTs thin film are on a 2 dimension space (placed parallel to the substrate and anode planes). The nanotubes cover the whole substrate with an average density of $10^{9} \mathrm{~cm}^{-2}$.
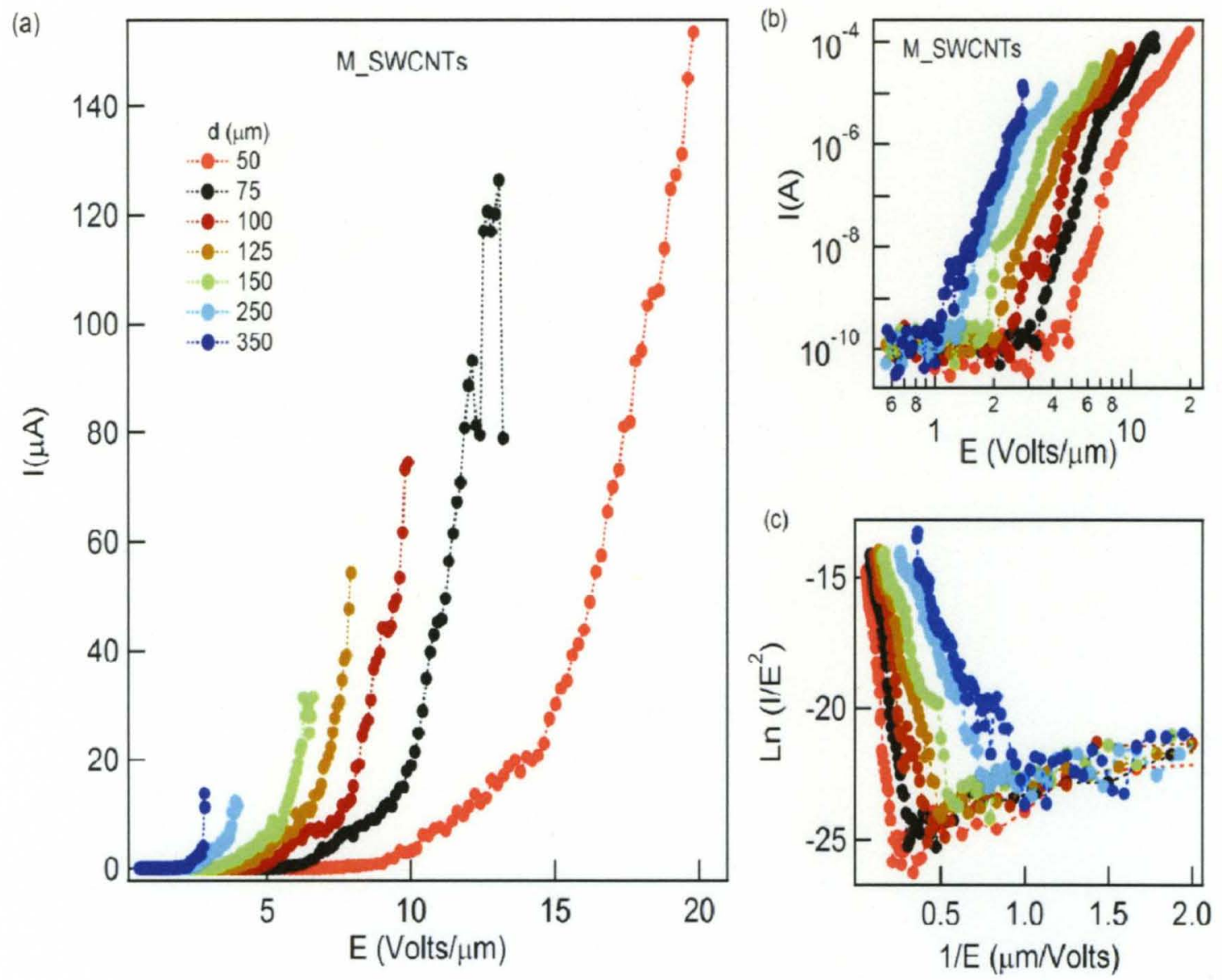

Figure 5-15: Emission current versus applied field in (a) linear scale (b) in Log scale (c) the corresponding F-N plot for M_SWCNTs. 
FE investigation of M_SWCNTs is conducted using the same conditions as those used for the previous nanostructures. The vacuum chamber is maintained at a pressure of $10^{-6}$ Torr. The voltage is swept from 0 to 1000 Volts, while the current is monitored using the Keithley 2400. The distance is varied during the interval $0-350 \mu \mathrm{m}$. The same procedure is followed to analyze the FE data taken for M_SWCNTs. The field enhancement factor, $\beta$, for M_SWCNTs ranges from 1000-5142 according to F-N analysis obtained from the slope of F-N plots.

Figure 5-15 shows the typical I-V characteristics of M_SWCNTs at different anode-cathode separations. The characteristics display good FE properties which includes low turn-on, threshold fields. The interval of the operating voltage is also small with good linearity of the corresponding F-N plots. The current density extracted from these samples is encouraging. At small $d$, the electric field is strong enough to extract large current, where it exceeds in some cases $260 \mu \mathrm{A}$. The linear scale shows clearly the effect of $d$ on the emission current and turn-on field while the logarithmic scale displays noticeably the emission saturation region with a lower slope than the other emitters. F-N analysis does apply to the I-V characteristics of M_SWCNTs where the linearity of F-N plots is visible at the emission current interval for each separation. There is a small instability in the emission current as depicted in the 3 curves which can be due to the presence of defects in the nanotubes or the non-uniformity of the film.

Overall, the as grown metallic CNTs (No attempts to optimize the film or the nanotubes themselves have been tried) are able to produce encouraging current density (up to $260 \mu \mathrm{A} / \mathrm{cm}^{2}$ ) further processing and development is needed to improve the efficiency of FE from metallic carbon nanotubes. 


\section{5-5. FE characterization of graphene}

Several graphene sheets are deposited on a silicon pillars for In-situ FE characterization.



Figure 5-16: SEM image of graphene sheets. ${ }^{139}$

Figure 5-16 shows a SEM image of a few graphene layers that are suspended with free edges that can be approached for FE characterization and a super tall and high density MWCNTs. The sample under investigation consists of about $500 \mathrm{~nm}$ long and 3 $\mathrm{nm}$ thick sheets which are electrostatically deposited on a conducting substrate. Zyvex nanomanipulator system is used for graphene manipulation. The graphene sheets are approached by the STM tip to collect the emitted electrons when the voltage is swept from 0 to 100 Volts at $\mathrm{d}=50 \mathrm{~nm}$. There are some challenges that are accompanying the manipulation of graphene for $I n$-situ characterization. 

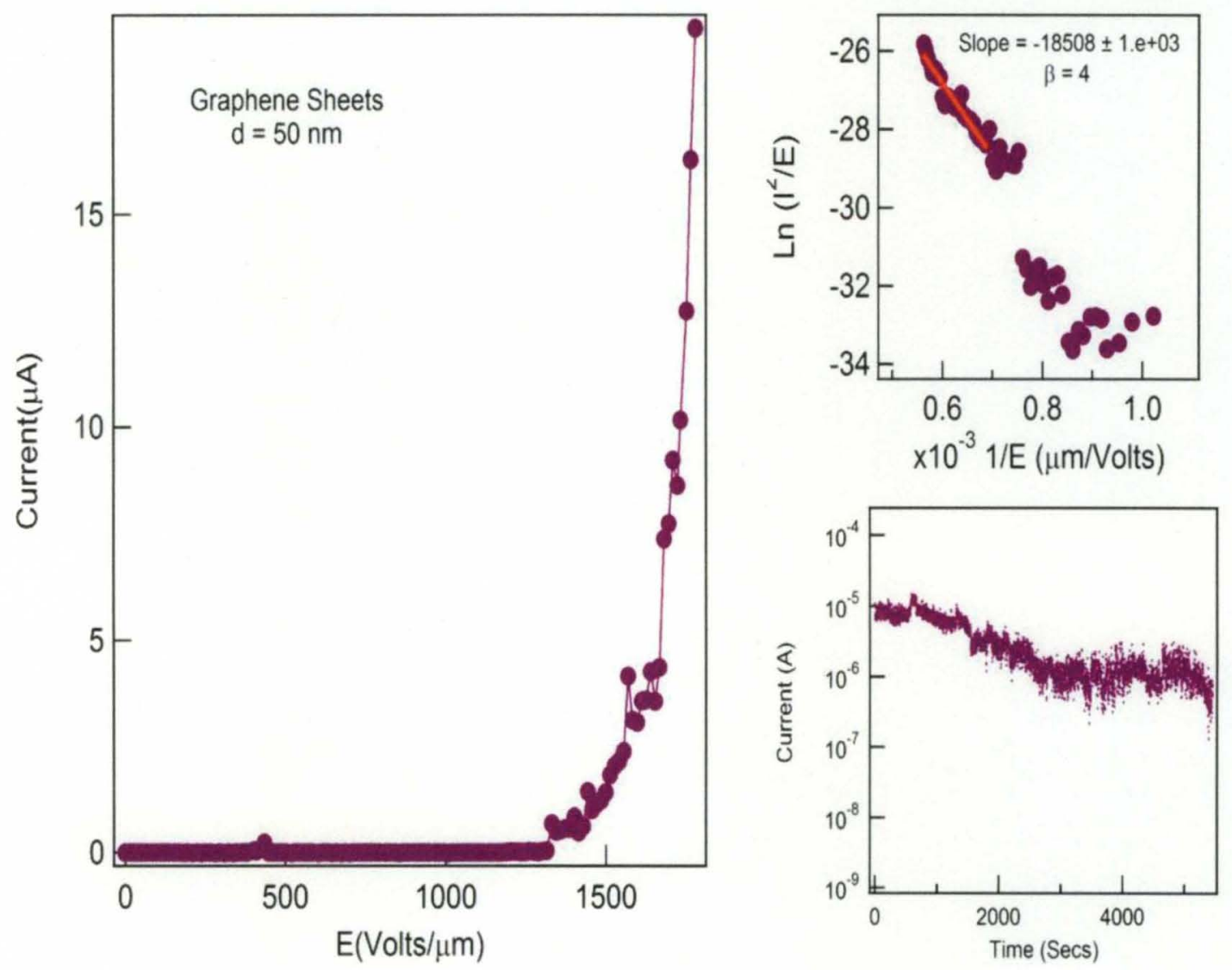

Figure 5-17: (a) I-E curves (b) the corresponding F-N plot for graphene (c) Emission current versus time for HOPG. ${ }^{140}$

Figure 5-17(a) and (b) shows the In-situ FE characterization of graphitic sheets. Figure 5-17(c) is the HOPG emission current versus time. Graphene sheets are characterized by a poor enhancement and very high turn-on field. Graphene also exhibits instability of emission which might be due to the poor mechanical stability of the sheet as they are suspended. The inferior FE properties of graphene is due to poor field enhancement $(\beta=4)$ and may be due to the existence of defects on the sheets investigated.

The FE from HOPG seems to decay during the first 30 minutes (Figure 5-17(c)) then starts to stabilize around $1 \mu \mathrm{A}$ with a fluctuation noise of $14 \%$. In addition, according 
to image spectroscopy of graphene sheets after FE, a distortion or damage can result while extracting electrons from these sheets due to arcing, thermal runaway or electrical discharge especially at very high eclectic fields.

In-situ FE characterization of graphene demonstrates poor properties while FE from HOPG proven to have an appreciable emission current corresponding to a density of $\sim 10$ $\mu \mathrm{A} / \mathrm{cm}^{2}$ and could produce a continuous emission current of $1 \mu \mathrm{A} / \mathrm{cm}^{2}$ for 2 hours. 


\section{5-6. Comparison of FE properties of CBNs}

Table 5- 4: Comparison of FE properties of CBN.

\begin{tabular}{|c|c|c|c|c|c|c|c|}
\hline Sample & $\begin{array}{c}\text { Density } \\
\left(108 / \mathbf{c m}^{2}\right)\end{array}$ & $\begin{array}{c}\text { Area } \\
\left(\mathbf{c m}^{2}\right)\end{array}$ & $\begin{array}{c}\text { Length } \\
(\mu \mathrm{m})\end{array}$ & $\begin{array}{c}\text { Radius } \\
(\mathbf{n m m})\end{array}$ & $\beta_{\max }$ & $\begin{array}{c}E_{T} \\
(\mathbf{V} / \mu \mathrm{m})\end{array}$ & $\begin{array}{c}I_{\max } \\
(\mu \mathrm{A})\end{array}$ \\
\hline CCNTs & 2.2 & 0.001 & 9 & 5 & 7600 & 0.66 & 520 \\
\hline MWCNTs & 30 & 2.25 & 10000 & 60 & 2000 & 1.5 & 2400 \\
\hline M_SWCNTs & 10 & 1 & & 10 & 1400 & 1.1 & 236 \\
\hline Graphene & $\begin{array}{c}\text { few } \\
\text { sheets }\end{array}$ & N/A & N/A & 0.1 & 4 & 1000 & 20 \\
\hline
\end{tabular}

Table 5-4 displays a comparison between the FE properties of CBNs. The comparison demonstrates that one dimensional CBNs (i.e. carbon nanotubes) are superior to the two dimensional nanostructures (graphene). Graphene exhibits the lowest FE properties with very feeble $\beta$, high turn-on electric field and low emission current. The highly dense emitter with all types should demonstrate very low FE properties, due to field screening, despite the size, the aspect ratio and the excellent intrinsic properties of the emitters.

Metallic CNT thin films demonstrate excellent FE properties over all the nanostructures investigated in the present study including the free standing 
semiconducting CNTs and CCNTs. The fact that thin film composed of laying on the substrate still have good $\beta$ that enhance emission by turning on the emission at $1.1 \mathrm{~V} / \mu \mathrm{m}$ and detectable current at higher $d$, means that field screening effect is significantly weak and that these structures and this technique can be adopted in many applications, local enhancement is present all over the sample. 


\section{CHAPTER VI}

\section{SUMMARY AND CONCLUSIONS}

In the present studies, electron emission microscopy has been employed to study the emission behavior of nanostructured materials. During the course of EE investigations a standardized method is established for FE and TE characterization using SEM, and subsequently the emitters' properties are modulated. In addition, an efficient system is designed for bulk EE investigation where the measurements could be optimized within a dedicated vacuum chamber.

The dependence of the applied electric field and temperature on the critical emission parameters for each emitter's material is the main focus of this research. The goal is to reveal the mechanism for EE from nanostructures with the hope to evaluate their future possible application in electron beam devices (i.e. displays, and traveling wave tubes) and energy conversion devices. EE from nanostructured carbon materials is then investigated under the application of an electric field or thermal excitation. Multiple structures and materials are evaluated and tested with the goal of finding the best electron emitters for both FE and TE. The most promising materials are carbon based nanoemitters since they combine electrical, mechanical, chemical and thermal properties along with an excellent local field enhancement allowing electron emission at low input energies (around few Volts $/ \mu \mathrm{m}$ and $500{ }^{\circ} \mathrm{C}$ ) which in turn prolongs the emitters' lifetime. 
One dimensional carbon based nanomaterials in the form of nanotubes and nanopipettes with their unique structural characteristics arising from their morphologies have been shown to exhibit enhanced field emission properties and support high current densities. Different CBNs samples with different densities, radii of curvatures, lengths and wall structures by varying the critical growth parameters are successfully synthesized. EE studies on these nanostructures resulted in a turn-on electric field as low as $0.7 \mathrm{~V} / \mu \mathrm{m}$, field enhancement factor as high as 7,600, turn-on temperatures of $500^{\circ} \mathrm{C}$ and high current densities. TE investigation is able to determine the work function of the emitting structures.

The present study shows that not only the large field amplification factor (arising from the small radius of curvature at the tip) enhanced the field emission properties, but also optimum emitter density plays an important role in reducing the field screening effect. In addition, the advantages of vacuum nanoelectronics as compared to the solid state electronics are confirmed and are as follows:

1. An exponential dependence of FE on the applied voltage, which permits the design of frequency converters and multipliers.

2. Low input energy is delivered to the nanostructures to produce considerable emission current.

3. No dissipation of energy as vacuum is the transport medium which can be needed for certain applications.

4. A high switching speed (lack of inertia) permits to design of high-frequency devices with short response time.

5. A high radiation tolerance and heat resistance of the CBNs emitters. 
As FE requires a high vacuum, and even ultra high vacuum, it is difficult to maintain the cleanness of the emitting tip due to the presence of contaminants either from the debris within the vacuum, or from diffusion of adsorbates into the emitting area. Carbon based nanomaterials can minimize this problem to a degree due to the fact that they are more resistant against rough vacuum environments. Image spectroscopy shows that CNT last longer and stay cleaner as compared to nanowires. The ability of nanostructures to support high current densities and resist tough environments such as thermal runaway, ion bombardment as well as surface modification makes them essential for future electron sources.

Embedded M_SWCNTs exhibit good EE properties through a competitive turnon field and onset temperature. This is can be attributed to their metallic behavior. Improvements on the structural design and surface treatment need to be addressed to improve the EE efficiency of these structures.

Nanostructured materials are the most pointed structures that can be employed in the EE field. They can produce the brightest and most monochromatic of electron emitter since their dimensions lie within tens of a nanometer. CBNs have reached few nanometers in diameter which allows the production of emitting spot smaller than $10^{-7} \mathrm{~cm}$ in its linear dimension.

FE based device can suffer from fast current fluctuation, producing considerable noise during operation. Stability and uniformity of emitters can reduce the noise level. Likewise, the nanostructures FE based device is not an exception; they also suffer the same problem. Additionally, FE based devices based device' lifetime is also an issue. Emitter's contamination by sputtering or ionized residual gasses can significantly 
decrease the emitter's lifetime and degrade device performance. Once optimized, CBNs can reduce and minimize the fluctuation noise and increase the emitter's lifetime.

TE displays the obvious disadvantage associated with the need of a heater in every single device. Depending on the cathode's material chosen, usually high temperature (greater than $1000{ }^{\circ} \mathrm{C}$ ) is needed to induce EE. The use of CBNs could decrease the turn $\mathrm{EE}$ temperature down to $500{ }^{\circ} \mathrm{C}$, moreover, treating these nanostructures can further reduce this value. Additionally, in the case of energy harvesting applications, this is not an issue as the heat energy is abundant by external sources, i.e. sun.

Table 6- 1 summarizes the EE properties obtained for nanostructured materials during the present work.

\begin{tabular}{|c|c|c|c|c|c|c|c|c|}
\hline \multirow[b]{2}{*}{ Material } & \multirow{2}{*}{$\begin{array}{c}\text { Density } \\
* 10^{8} \\
\mathrm{~cm}^{-2}\end{array}$} & \multicolumn{3}{|c|}{ Field Emission } & \multicolumn{4}{|c|}{ Thermionic Emission } \\
\hline & & $\boldsymbol{\beta}_{\max }$ & $\begin{array}{c}\mathbf{E}_{\mathrm{T}} \\
\mathbf{V} / \mu \mathrm{m}\end{array}$ & $\begin{array}{c}\mathbf{J}_{\mathrm{FE}} \\
\mu \mathrm{A} / \mathrm{cm}^{2}\end{array}$ & $\begin{array}{c}\mathrm{J}_{\text {FETE }} \\
\mu \mathrm{A} / \mathrm{cm}^{2}\end{array}$ & $\begin{array}{c}\mathbf{J}_{\mathrm{TE}} \\
\mu \mathrm{A} / \mathrm{cm}^{2}\end{array}$ & $\begin{array}{l}\mathbf{T}_{\mathrm{T}} \\
{ }^{0} \mathrm{C}\end{array}$ & $\begin{array}{l}\Phi \\
\mathrm{eV}\end{array}$ \\
\hline CCNTs & 2.2 & 7600 & 0.66 & $520 * 10^{3}$ & 160 & 30 & 900 & 4.2 \\
\hline MWCNTs & 30 & 2000 & 1.5 & $1.06 * 10^{3}$ & 13 & 4 & 1000 & 4.8 \\
\hline $\begin{array}{l}\text { Metallic } \\
\text { SWCNTs }\end{array}$ & 10 & 1400 & 1.1 & 236 & 515 & 100 & 500 & 4.56 \\
\hline Graphene & $\begin{array}{c}\text { Few } \\
\text { sheets }\end{array}$ & 4 & 1000 & $\mathrm{I}=20 \mu \mathrm{A}$ & & & & \\
\hline W_NWs & 20 & 500 & 5 & 10 & & & & \\
\hline $\begin{array}{c}\mathrm{Ag}_{2} \mathrm{Ga} \\
\mathrm{NWs}\end{array}$ & $\begin{array}{c}\text { Single } \\
\text { NW }\end{array}$ & & N/A & & & & & \\
\hline
\end{tabular}

Table 6- 2 summaries the outcome of characterizing EE from nanostructured materials. 


\begin{tabular}{|c|c|c|c|c|}
\hline Material & Knowledge learned & Advantages & Disadvantages & Questionable results \\
\hline CCNTs & $\begin{array}{l}\text { Tapered nanostructures } \\
\text { and well spaced emitters } \\
\text { produce better EE } \\
\text { Morphology of an emitter } \\
\text { influence the EE } \\
\text { performance }\end{array}$ & $\begin{array}{l}\text { High aspect ratio } \\
\text { High field } \\
\text { enhancement } \\
\text { Tapered structure }\end{array}$ & $\begin{array}{l}\text { Grown only on a Pt- } \\
\text { cylindrical substrate } \\
\text { or on a graphite foil }\end{array}$ & $\begin{array}{l}\text { Conductivity } \\
\text { Current increase in the } \\
\text { TE saturation region, } \\
\text { Source behind change in } \\
\text { the F-N plot's slope }\end{array}$ \\
\hline MWCNTs & $\begin{array}{l}\text { High emitters' density } \\
\text { produce poor EE }\end{array}$ & $\begin{array}{l}\text { High aspect ratio } \\
\text { Super high field } \\
\text { enhancement } \\
\text { Can be grown on } \\
\text { different substrates } \\
\text { Capped/opened end }\end{array}$ & N/A & $\begin{array}{l}\text { Increase in the } \\
\text { background FE current } \\
\text { (when the voltage is } \\
\text { turned-on) as the } \\
\text { distance decreases }\end{array}$ \\
\hline $\begin{array}{l}\text { Metallic } \\
\text { SWCNTs }\end{array}$ & $\begin{array}{l}\text { Metallic behavior of CNTs } \\
\text { improves EE }\end{array}$ & $\begin{array}{l}\text { Metallic } \\
\text { High aspect ratio } \\
\text { Can be dispersed on } \\
\text { different substrate }\end{array}$ & Lateral growth (film) & $\begin{array}{l}\text { Source of emission } \\
\text { Start-up current }\end{array}$ \\
\hline Graphene & $\begin{array}{l}\text { Two-dimensional } \\
\text { nanostructures are weak } \\
\text { field emitters } \\
\text { Multiple uniform graphene } \\
\text { sites can improve EE }\end{array}$ & $\begin{array}{l}\text { Resist against rough } \\
\text { environments } \\
\text { Nano-sites }\end{array}$ & $\begin{array}{l}\text { Very low field } \\
\text { enhancement }\end{array}$ & $\begin{array}{l}\text { Source of emission: } \\
\text { Zigzag or armchair }\end{array}$ \\
\hline $\begin{array}{l}\text { W- } \\
\text { NWs }\end{array}$ & $\begin{array}{l}\text { Better conductivity and } \\
\text { smaller radius enhance EE }\end{array}$ & High aspect ratio & $\begin{array}{l}\text { Low conductivity } \\
\text { Low melting } \mathrm{T}\end{array}$ & Exact melting current \\
\hline $\begin{array}{l}\mathrm{Ag}_{2} \mathrm{Ga}- \\
\text { NWs }\end{array}$ & $\begin{array}{l}\text { Good thermal and electrical } \\
\text { properties are essential for } \\
\text { good EE cathodes }\end{array}$ & High aspect ratio & $\begin{array}{l}\text { Low conductivity } \\
\text { Low melting } \mathrm{T}\end{array}$ & $\begin{array}{l}\text { The exact value of the } \\
\text { melting current }\end{array}$ \\
\hline
\end{tabular}

In the present work, the development and application of high power electron point sources are presented. With controlled aspect ratio, density, and uniformity; CNTs can be turned into potential and next generation field emitters. It can be concluded that this work will contribute to the electron beam technology especially in the area of SEM columns, nanomachining and energy conversion. 


\section{REFERENCES}

3

4

5

6

7

8

D. M. Goebel, Y. Hirooka, and G. A. Campbell, Review of Scientific Instruments 56, 1888 (1985).

10

D. W. Tuggle and L. W. Swanson, Journal of Vacuum Science \& Technology B: Microelectronics and Nanometer Structures 3, 220 (1985).

11

L. Erik, Tungsten: Properties, Chemistry, Technology of the Element, Alloys, and Chemical Compounds, 1 ed. (Springer, 1999). 
G. Fursey, Field Emission In Vacuum Microelectronics (Kluwer Academic and Plenum Publishers, New York, 2003).

O. Groning, O. M. Kuttel, C. Emmenegger, P. Groning, and L. Schlapbach, Journal of Vacuum Science \& Technology B: Microelectronics and Nanometer Structures 18, 665 (2000).

L. Nilsson, O. Groening, C. Emmenegger, O. Kuettel, E. Schaller, L. Schlapbach, H. Kind, J. M. Bonard, and K. Kern, Applied Physics Letters 76, 2071 (2000).

J.-M. Bonard, N. Weiss, H. Kind, T. Stöckli, L. Forró, K. Kern, and A. Châtelain, Advanced Materials 13, 184 (2001).

C. A. Spindt, Journal of Applied Physics 39, 3504 (1968).

W. P. Kang, J. L. Davidson, K. Subramanian, B. K. Choi, and K. F. Galloway, Nuclear Science, IEEE Transactions on 54, 1061 (2007).

W. Zhu, Vacuum Microelectronics 1ed. (Wiley-Interscience, 2001).

W. J. Orvis, C. F. McConaghy, D. R. Ciarlo, J. H. Yee, and E. W. Hee, Electron Devices, IEEE Transactions on 36, 2651 (1989).

P. W. Hawkes, Advances In Electronics And Electron Physics V83 (Academic press., 1992).

G. N. Fursey, D. V. Novikov, G. A. Dyuzhev, A. V. Kotcheryzhenkov, and P. O. Vassiliev, Applied Surface Science 215, 135 (2003).

N. de Jonge and J. M. Bonard, Philosophical Transactions of the Royal Society of London. Series A: Mathematical, Physical and Engineering Sciences 362, 2239 (2004).

S. Raghunandan and et al., Nanotechnology 19, 065605 (2008). 
A. W. Denier van der Gon, M. F. F. K. Jongen, H. H. Brongersma, U. van Slooten, and A. Manenschijn, Applied Surface Science 111, 64 (1997).

J. Orloff, L. W. Swanson, and J.-Z. Li, Journal of Vacuum Science \& Technology B: Microelectronics and Nanometer Structures 3, 224 (1985).

N. Hamada, S.-i. Sawada, and A. Oshiyama, Phys. Rev. Lett. 68, 1579 (1992).

J. W. G. Wilder, L. C. Venema, A. G. Rinzler, R. E. Smalley, and C. Dekker, Nature 391, 59 (1998).

C. A. Spindt, I. Brodie, L. Humphrey, and E. R. Westerberg, J. Appl. Phys. 47, 5248 (1976).

W. B. Choi, D. S. Chung, J. H. Kang, H. Y. Kim, Y. W. Jin, I. T. Han, Y. H. Lee, J. E. Jung, N. S. Lee, G. S. Park, and J. M. Kim, Applied Physics Letters 75, 3129 (1999).

R. T. Olson, G. R. Condon, J. A. Panitz, and P. R. Schwoebel, Journal of Applied Physics 87, 2031 (2000).

Y. Gogotsi, NANOMATERIALS HANDBOOK (CRC, 2006).

B. W. Han, H. S. Lee, and B. T. Ahn, Applied Surface Science 187, 45 (2002). 
C.-M. Lin, S.-J. Chang, M. Yokoyama, F.-Y. Chuang, C.-H. Tsai, W.-C. Wang, and I.-N. Lin, Jpn. J. Appl. Phys. 38, 890 (1999).

M. M. J. Treacy, T. W. Ebbesen, and J. M. Gibson, Nature 381, 678 (1996).

E. W. Wong, P. E. Sheehan, and C. M. Lieber, Science 277, 1971 (1997).

M. R. Falvo, G. J. Clary, R. M. Taylor, V. Chi, F. P. Brooks, S. Washburn, and R. Superfine, Nature 389, 582 (1997).

S. Berber, Y.-K. Kwon, and D. Tománek, Physical Review Letters 84, 4613 (2000). Deng, Physical Review B (Condensed Matter and Materials Physics) 73, 235412 (2006).

Z. Kaiwang and et al., Nanotechnology 18, 285703 (2007).

P. G. Collins and P. Avouris, Scientific American 96, 283(6) (2000).

F. Jin, Y. Liu, C. M. Day, and S. A. Little, Journal of Vacuum Science \& Technology B: Microelectronics and Nanometer Structures 25, 1785 (2007).

E. L. Murphy and R. H. Good, Physical Review 102, 1464 (1956).

J. Harris and R. O. Jones, Journal of Physics C: Solid State Physics, 3751 (1974).

C. Kittel, Introduction To Solid State Physics (John Wiley \& Sons, 2005).

L. L. Barnes, Physical Review 42, 487 (1932).

O. W. Richardson, Physical Review 23, 153 (1924).

S. Seely, Physical Review 59, 75 (1941).

P. Liu, Y. Wei, K. Jiang, Q. Sun, X. Zhang, S. Fan, S. Zhang, C. Ning, and J. 
A. Safir, R. W. Cohn, Unpublished work.

S. Zaima, H. Adachi, and Y. Shibata, Journal of Vacuum Science \& Technology B: Microelectronics and Nanometer Structures 2, 73 (1984).

55 L. W. Nordheim, Proceedings of the Royal Society of London. Series A, Containing Papers of a Mathematical and Physical Character 121, 626 (1928).

R. H. Fowler and L. Nordheim, Proceedings of the Royal Society of London. Series A, Containing Papers of a Mathematical and Physical Character 119, 173 (1928).

57 V. Semet, C. Adessi, T. Capron, R. Mouton, and V. Thien Binh, Physical Review B 75, 045430 (2007).

R. B. Marcus, T. S. Ravi, T. Gmitter, H. H. Busta, J. T. Niccum, K. K. Chin, and D. Liu, Electron Devices, IEEE Transactions on 38, 2289 (1991). (2008).

A. Safir, R. W. Cohn, Unpublished work.

A. Safir, R. W. Cohn, Unpublished work.

L. R. Koller, Physical Review 25, 671 (1925). 
G. Gärtner, P. Geittner, H. Lydtin, and A. Ritz, Applied Surface Science 111, 11 (1997).

G. W. Billings, (1999).

S. Otani, H. Hiraoka, M. Ide, and Y. Ishizawa, Journal of Alloys and Compounds 189, L1 (1992).

M.-C. Kan, J.-L. Huang, J. C. Sung, K.-H. Chen, and B.-S. Yau, Carbon 41, 2839 (2003).

F. Jin, Y. Liu, and C. M. Day, Applied Physics Letters 88, 163116 (2006).

X. Xi, Z. Nie, W. Wang, J. Yang, S. Hao, Y. Guo, and T. Zuo, Applied Surface Science 251, 134 (2005).

G. H. M. Gubbels, L. R. Wolff, and R. Metselaar, Journal of Applied Physics 64, 1508 (1988).

F. Zocchi, Applied physics letters 47, 1276 (1985).

M. E. Herniter and W. D. Getty, IEEE Transactions On Plasma Science 18, 992 (1990).

M. Futamoto, M. Nakazawa, K. Usami, Hosoki, and U. Kawabe, J. Appl. Phys. 51, 3869 (1980).

Y. Liu, C. M. Day, S. A. Little, and F. Jin, Journal of Vacuum Science \& Technology A: Vacuum, Surfaces, and Films 24, 2187 (2006). Auciello, Physical Review B 72, 165404 (2005). 

AS THERMIONIC EMITTERS, Albuquerque, New Mexico (USA), 2004 (AIP), p. 773.

81 T. Fujii and M. Ohta, Journal of Physics D: Applied Physics 28, 1268 (1995).

A. Safir, R. W. Cohn, Unpublished work.

A. Safir, R. W. Cohn, Unpublished work.

A. Safir, R. W. Cohn, Unpublished work.

W. I. Milne, A. Ilie, J. B. Cui, A. Ferrari, and J. Robertson, Diamond and Related Materials 10, 260 (2001).

R. Krupke, F. Hennrich, H. v. Lohneysen, and M. M. Kappes, Science 301, 344 (2003). 

4, 317 (2004).

L. Qu, F. Du, and L. Dai, Nano Letters 8, 2682 (2008).

T. Paronyan, G. U. Sumanasekera

A. Sidorov, D. Mudd, G. Sumanasekera, P. J. Ouseph, C. S. Jayanthi, and S.-Y. Wu, Nanotechnology 20, 055611 (2009).

S. Pabba, A. Safir, R. W. Cohn, Unpublished work.

M. M. Yazdanpanah, Thesis, University of Louisville, 2006.

M. M. Yazdanpanah, S. A. Harfenist, A. Safir, and R. W. Cohn, Journal of Applied Physics 98, 073510 (2005).

J. Thangala, S. Vaddiraju, R. Bogale, R. Thurman, T. Powers, B. Deb, and Mahendra K. Sunkara, Small 3, 890 (2007).

A. Safir, R. W. Cohn, Unpublished work.

103

C. J. Chen, Introduction To Scanning Tunneling MicroscopY (Oxford University Press, 1993).

A. Safir, R. W. Cohn, Unpublished work.

105

A. Safir, G. U. Sumanasekera, R. W. Cohn, Unpublished work.

106

A. Safir, G. U. Sumanasekera, R. W. Cohn, Unpublished work.

107

A. Safir, R. W. Cohn, unpublished work

108

S. Dumpala, A. Safir, D. Mudd, R. W. Cohn, M. K. Sunkara, and G. U. Sumanasekera, Diamond and Related Materials 18, 1262 (2009). 
H. Ago, T. Kugler, F. Cacialli, W. R. Salaneck, M. S. P. Shaffer, A. H. Windle, and R. H. Friend, The Journal of Physical Chemistry B 103, 8116 (1999).

J. P. Sun, Z. X. Zhang, S. M. Hou, G. M. Zhang, Z. N. Gu, X. Y. Zhao, W. M. Liu, and Z. Q. Xue, Applied Physics A: Materials Science \& Processing 75, 479 (2002).

M. Shiraishi and M. Ata, Carbon 39, 1913 (2001).

Unpublished work, Data provided by S. Dumpala

Unpublished work. Samples provided by G. U. Sumanasekera and images taken by A. Safir

114 Samples are provided by G. U. Sumanasekera

A. Safir, D. Mudd, T. Paronyan, G. Sumansukara, and R. W. Cohn, (2010).

Y. Kojima, K. Kasuya, T. Ooi, K. Nagato, K. Takayama, and M. Nakao, Jpn. J. Appl. Phys. 46, 6250 (2007).

J. Liu, Z. Zhang, Y. Zhao, X. Su, S. Liu, and E. Wang, Small 1, 310 (2005).

Z. Chen, Y. Wei, C. X. Luo, K. L. Jiang, L. Zhang, Q. Q. Li, S. S. Fan, and J. C. Gao, Applied Physics Letters 90, 3 (2007). T. Hirao, and K. Oura, Applied Physics Letters 90, 153108 (2007).

W. I. Milne, K. B. K. Teo, G. A. J. Amaratunga, P. Legagneux, L. Gangloff, J. P. Schnell, V. Semet, V. T. Binh, and O. Groening, Journal of Materials Chemistry 14, 933 (2004). 893 (2001). 
J.-M. Bonard, J.-P. Salvetat, T. Stockli, W. A. de Heer, L. Forro, and A. Chatelain, Applied Physics Letters 73, 918 (1998).

Q. H. Wang, T. D. Corrigan, J. Y. Dai, R. P. H. Chang, and A. R. Krauss, Applied Physics Letters 70, 3308 (1997).

M. Sveningsson, R. E. Morjan, O. A. Nerushev, Y. Sato, J. Bäckström, E. E. B. Campbell, and F. Rohmund, Applied Physics A: Materials Science \& Processing 73, 409 (2001).

A. Safir, R. W. Cohn, unpublished work

A. Safir, R. W. Cohn, unpublished work

G. Zhang, X. Jiang, and E. Wang, Science 300, 472 (2003).

R. D. Lowe, R. C. Mani, R. P. Baldwin, and M. K. Sunkara, Electrochemical and Solid-State Letters 9, H43 (2006).

W. Zhang, Z. H. Xi, G. M. Zhang, S. Wang, M. S. Wang, J. Y. Wang, and Z. Q. Xue, Applied Physics A: Materials Science \& Processing 86, 171 (2007). Physics Letters 87, 143107 (2005).

M.-S. Wang, Q. Chen, and L.-M. Peng, Small 4, 1907 (2008).

K. A. Dean, T. P. Burgin, and B. R. Chalamala, Applied Physics Letters 79, 1873 (2001). Deposition 14, 256 (2008). Physics D: Applied Physics 34, 1597 (2001). 
136 A. Maiti, J. Andzelm, N. Tanpipat, and P. von Allmen, Phys Rev Lett 87, 155502 (2001).

137 A. Safir, R. W. Cohn, unpublished work

138 A. Safir, R. W. Cohn, unpublished work

139 A. Safir, R. W. Cohn unpublished work

140

A. Safir, R. W. Cohn, unpublished work 


\section{APPENDIXES}

\section{Application of FE from nanostructures for nanomachining:}

\section{Deformation and Localized Melting of Gold and Tungsten Tips}

Nanomachining of metals is conducted under the same experimental conditions as those used for FE experiments. A single or bundle of CCNTs approaches a sharp tungsten STM tip or a dull gold microwire. The inter-electrode distance is kept small in the order of few microns and the FE process is turned on while the SEM beam blanker is turned off.

\section{Melting of Tungsten}

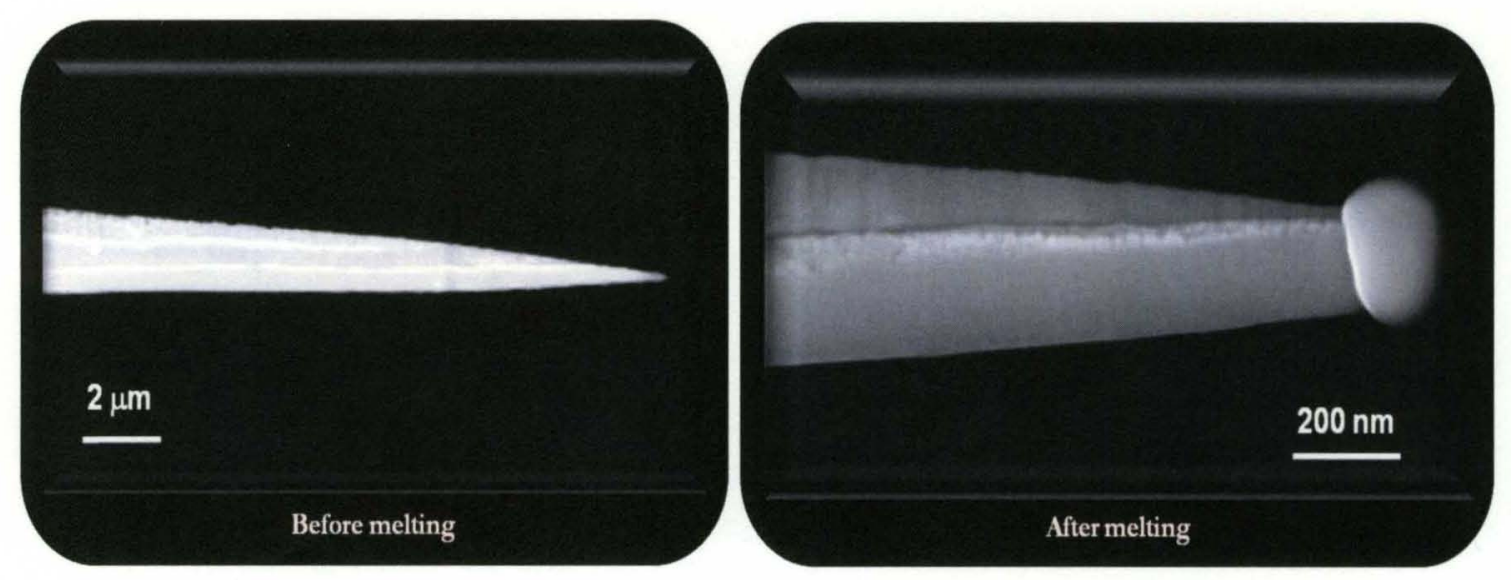

Figure 1: Time-lapse SEM image of melting of tungsten STM tip.

Figure 1 shows the tungsten STM tip before and after FE takes place. A nanovolume is able to form immediately after FE turns on. 


\section{Melting of Gold}



Figure 2: Time-lapse SEM images of melting of gold microwire. ${ }^{*}$

Figure 2 shows gold microwire, which is manually sharpened, before and after been exposed to two successive FE current of a few micrometers. A nano-volume is able to form immediately after FE turns on. Since the melting temperature of gold is almost half of that of tungsten, the gold melting of a micro-sized gold volume is easier. As it will be demonstrated, the resulting melting current and time is smaller in case of gold. 


\section{Deformation of Tungsten}

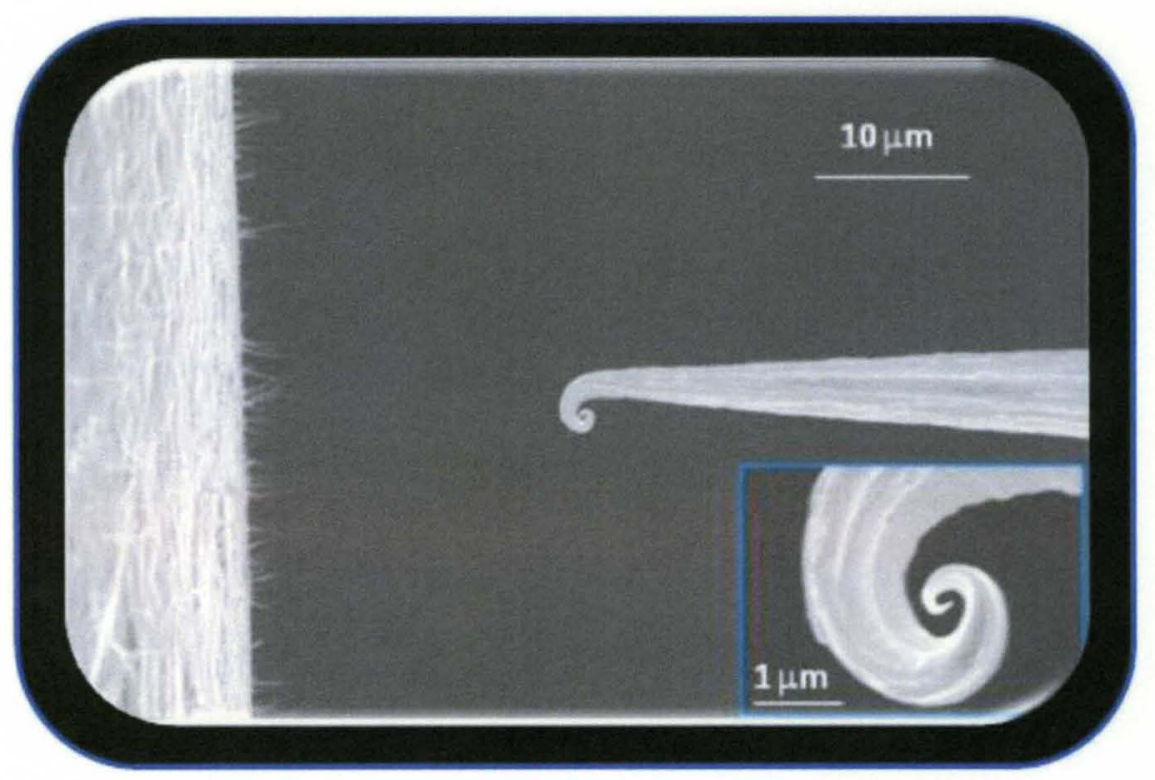

Figure 3: Field emission induced deformation of STM tip. ${ }^{(*)}$

Field emission based technique is used to melt and deform different types of metals. Tungsten for instance, which have a high melting point of $3656 \mathrm{~K}$ can be deformed and melted by electron beam emitted from a single CCNT. Nano-volume is able to form by applying an emission current of 1-5 $\mu \mathrm{A}$ in just few seconds. Likewise gold microwire is manually sharpened down to sub-100 hundred nanometers and is melted using FE current that is one order of magnitude smaller and in shorter time that in the case of tungsten STM tip. This result is obtained while the SEM electron beam is off. Nevertheless, the contribution of the e-beam can't be ignored since the sample needs to be imaged and placed at a close proximity to the STM probe before the melting process takes place. 


\section{The Melting Current and Time Estimations}

To estimate the minimum time and the current required to melt a nano-volume of tungsten, one needs to use the conservation of energy principle. It is assumed that the energy loss through thermal radiation and heat transfer (via conduction along the wire) is negligible.

The tungsten tip takes a conical shape; its summit is exposed to the electron beam extracted from single CCNT. The current $(I)$ induces the heating and caused a partial melting at the tungsten tip.

The equation of conservation of energy states that the energy delivered $\left(P_{d}\right)$ is equal to the energy transferred $\left(P_{t}\right)$ minus any meaningful lost. The equations below are used to derive the current and time to melt a nano-volume of tungsten:

$$
\begin{aligned}
& P_{d}=I^{2} R \\
& P_{t}=c m \Delta T
\end{aligned}
$$

As a result,

$$
\begin{gathered}
P_{d}=P_{t} \\
I^{2} \frac{\rho_{0}(\alpha \Delta T+1)}{\pi(\tan \theta)^{2}}\left(\frac{1}{L_{0}}-\frac{1}{L}\right)=c * D * \frac{\pi}{3}\left(R^{2}{ }_{1}+R_{l} R_{2}+R_{2}^{2}\right) \frac{\Delta T}{t}
\end{gathered}
$$

Where,

$$
\begin{aligned}
& P_{t}=c m \frac{\Delta T}{t} \\
& R=\frac{l}{A} \rho=\frac{l}{A} \rho_{0}(\alpha \Delta T+1)
\end{aligned}
$$




$$
\begin{aligned}
& R=\frac{\rho_{0}(\alpha \Delta T+1)}{\pi(\tan \theta)^{2}}\left(\frac{1}{L_{0}}-\frac{1}{L}\right) \\
& A=\pi(l \tan \theta)^{2} \\
& m=\text { density } * \text { volume }=D * \frac{\pi}{3}\left(R^{2}{ }_{1}+R_{l} R_{2}+R^{2}{ }_{2}\right) \\
& \Delta T=T_{m}-T_{R}
\end{aligned}
$$

$c$, is the specific heat capacity, $D$ is the density, $\rho_{0}$ is the electrical resistivity and $\alpha$ is the thermal expansion coefficient of tungsten. $L_{0}$ is the initial length of the cone; $L$ is the length of the remaining portion of the cone. $R_{1}$ and $R_{2}$ are the radii of the cone's base and top. $\theta$ the cone angle (Figure 4$). T_{R}$ and $T_{m}$ are the room and melting temperature of tungsten.

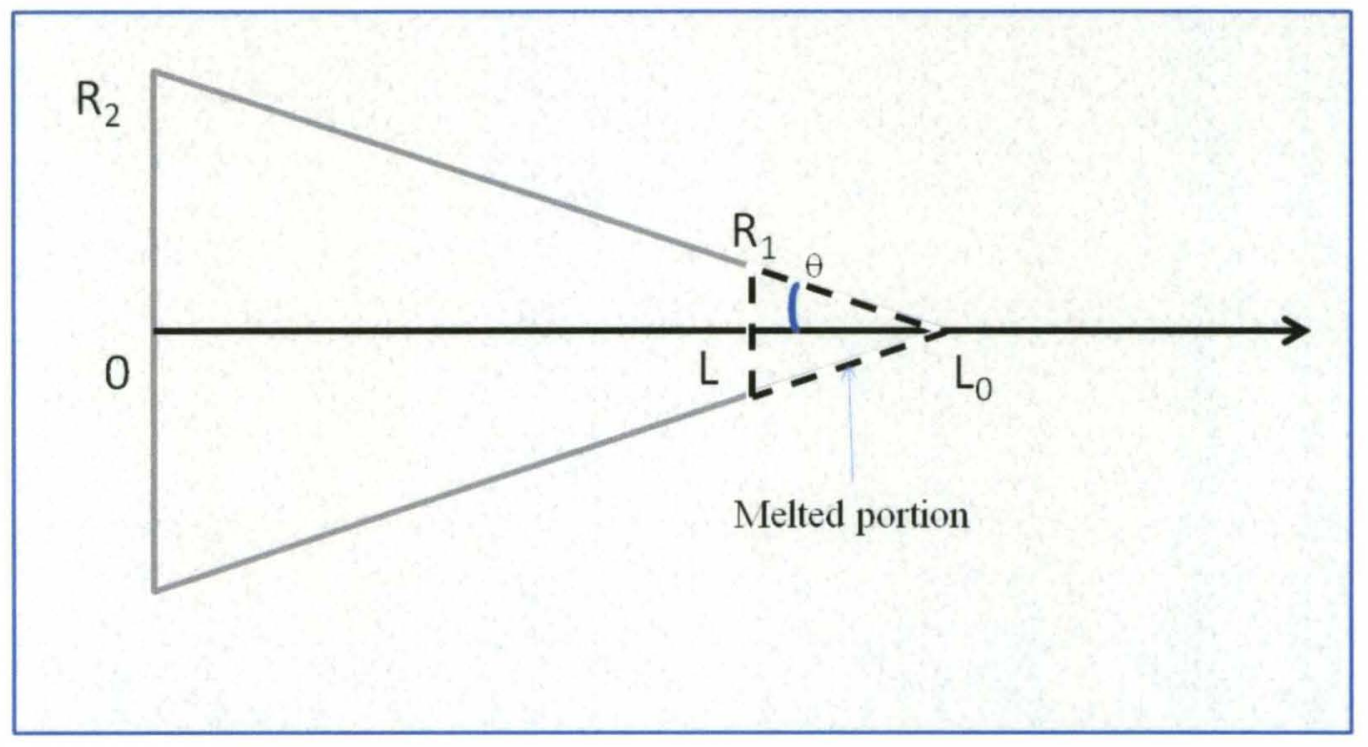

Figure 4: 2-d schematic of the tungsten tip..$^{(*)}$

Solving equation 4 for the current and temperature results in the curves sown on Figure 4. Figure 4 is the FE current versus time required to melt a nano-volume of tungsten or gold. 


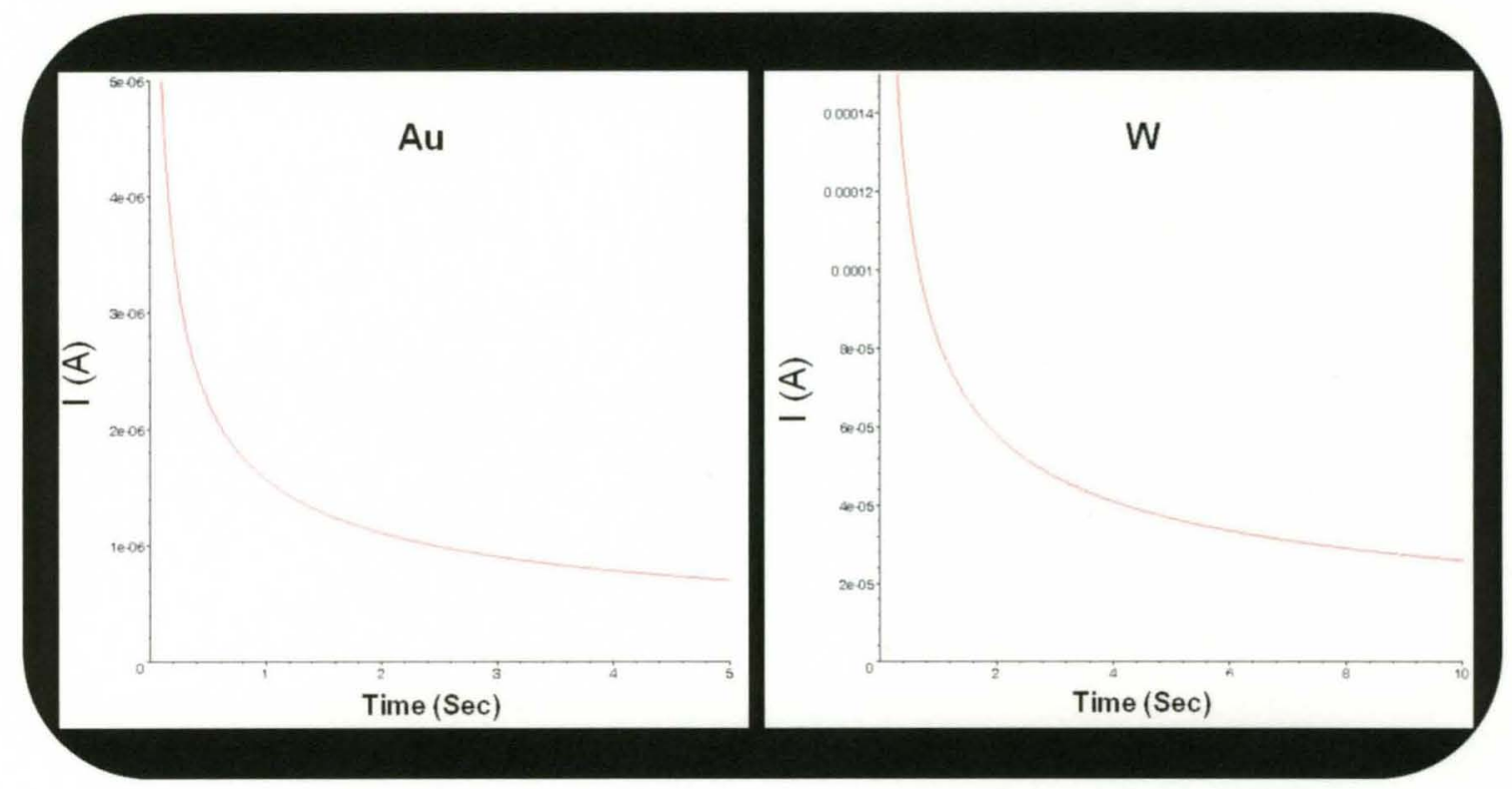

Figure 5: Variation of the melting current as a function of time for gold and tungsten tips. ${ }^{(*)}$

The numerical values of the physical quantities used to derive the melting current for tungsten are as follow:

$$
\begin{aligned}
& c=140 \mathrm{~J} / \mathrm{Kg} \cdot \mathrm{K} \\
& D=19250 \mathrm{Kg} \cdot \mathrm{m}^{2} \\
& \rho=5 * 10^{-8} \Omega \cdot \mathrm{m} \\
& \alpha=0.0045 \\
& \Delta T=3395 \mathrm{~K} \\
& \theta=19.7^{\circ} \\
& L=3.3 \mu \mathrm{m} \\
& L_{0}=0.85 \mu \mathrm{m} \\
& R_{1}=145.5 \mathrm{~nm} \\
& R_{2}=626.5 \mathrm{~nm}
\end{aligned}
$$

${ }^{(*)}$ A. Safir, R. W. Cohn, Unpublished work. 


\section{CURRICULUM VITAE ABDELILAH SAFIR \\ 323 Ridgedale Rd \#3 Louisville, KY40206}

\section{EDUCATION}

University of Louisville, Louisville, KY

$\begin{array}{lll}\text { Ph.D. GPA: } 3.87 \quad \text { Electrical Engineering, } & 2010\end{array}$

Future Professor Program Certificate Education 2004

$\begin{array}{lll}\text { M.S. GPA: } 3.7 & \text { Physics } & 2002\end{array}$

Hassan II University, Casablanca, Morocco

$\begin{array}{lll}\text { M.S } & 1999\end{array}$

\section{EMPLOYMENT EXPERIENCE}

CONSULTING SERVICES, $\quad 01 / 09-$ date

Nauganeedles LLc. $\quad$ Louisville, KY

- Developed specialized and novel processes for nanostructures applications.

- Contributed to the fabrication of a super high aspect ratio (> 1000) AFM probes.

- Synthesized intermetallic nanostructures at room temperature (Bulk and In-Situ growth).

- Developed a new electrochemical etching technique for nanoprobe fabrication.
GRADUATE RESEARCH ASSISTANT,
$08 / 02$ - date

ElectroOptics Research Institute and Nanotechnology Center, Louisville, KY

- Characterized novel nano-emitters as electron point sources for energy conversion applications: "i.e. Metallic and semiconductor CNT, graphene, diamond"

- Nanolithography using AFM (Feature size as small as $10 \mathrm{~nm}$ were achieved).

- Expert in nanoimprint lithography using hot and UV nano embossing.

- Able to perform force spectroscopy and rheological properties on complex structures (i.e. polymeric nanofibers).

- Fabricated and characterized nanoelectronic devices.

- Capable of maintaining state-of-the-art spectroscopy equipments for surface analysis.

- Able to train and supervise incoming employees and students from different levels and backgrounds.

GRADUATE TEACHING ASSISTANT,

2000-2002

University of Louisville, Louisville, KY

- Taught physics to engineering, medical and physics undergraduate students.

- Tutored courses in the engineering, mathematics, physics and chemistry.

- Participated in the evaluation process of the incoming physics professors. 


\section{SKILLS}

\section{LABORATORY AND INSTRUMENTATION SKILLS}

Thin Film Characterization: Atomic Force Microscopy (AFM), Scanning Electron Microscopy (SEM), Spectroscopic Ellipsometry, Inferometry, Energy Dispersive XRay Spectroscopy (EDS), X-Ray Powder Diffraction (XRD), X-ray Photoelectron spectroscopy (XPS), Raman spectroscopy. Spectral power distributions measurements.

Micro/Nano Fabrication Processes: Metallization, RF/DC Sputtering, E-beam Evaporation, Thermal evaporation, Spray Pyrolysis, Electroplating, Electrochemical deposition, Chemical deposition, Electrospinning, Optical and E-Beam Lithography, Liftoff, Electrochemical Etching, Wet/dry chemical etching, Wet/dry oxidation, Wafer doping, Sawing, Polishing, and Micro/nanopipettes fabrication.

Nano-Material Synthesis: Hot-Filament Chemical Vapor Deposition (HF-CVD), Plasma Enhanced Chemical Vapor Deposition (PE-CVD) and Self-assembly processes.

Material Analysis \& Characterization: Magnetic Force Microscopy (MFM), Conductive AFM (C-AFM), thermal AFM, Force Modulation Microscopy, ForceDistance Measurements Nanoindenting/Scratching, Phase imaging, Spectroscopic Ellipsometry, Inferometry, Raman spectroscopy, and Spectral power distributions measurements.

Transport Properties Measurement: Electrical (IV, Gate dependence, 2/4 probe Conductivity), Mechanical (Force-distance curves), Thermal (Thermo-power and thermal conductivity), Magnetic (Magneto-resistance, Hall Effect) and Electrochemical (Cyclic Voltammetry) measurements techniques.

\section{SOFTWARE SKILLS}

Image analysis software (SPIP), Microsoft Office, Igor, Maple, L-Edit, Photoshop, Labview, AutoCAD, Windows XP/Vista/Windows7.

\section{LANGUAGES}

Fluent in Arabic, English and French.

\section{PROFESSIONAL SOCIETIES}

American Physical Society (APS) and Institute of Electrical and Electronic Engineers (IEEE).

\section{AWARDS, HONORS AND RECOGNITIONS}

- Graduate Research Assistantship, Electrical \& Computer Engineering Department, University of Louisville, 2002-2010.

- Recognition by University of Louisville for research leading to commercialization, 2009.

- Listed in Who is Who in American Universities and Colleges, 2008. 
- Nanotechnology 19 035502: Recognized as a featured and part of the top $10 \%$ popular article, 2008.

- Second Place Winner Promotional/Poster Section, International Material Nanotechnology Workshop, Lexington, Kentucky, 2004.

- Graduate Teaching Assistantship, Physics Department, University of Louisville, 2000-2002.

PATENTS AND INVENTIONS (One provisional U.S. Patent application submitted June 2007).

Title: Metallic nanostructures self-assembly, and testing methods.

\section{PROFESSIONAL ACTIVITIES AND SERVICES}

Voluntary Physics Teacher at University of Louisville

2003-2004

Science fair judge at DuPont Manual High School, Louisville, KY 2001

Physics Tutor at the Physics-Learning Center, Louisville, KY 2002

\section{REFEREED PUBLICATIONS}

1. Abdelilah Safir, David Mudd, Santoshrupa Dumpala, Mahendra K. Sunkara, Robert W. Cohn, and Gamini U. Sumanasekera; "Thermionic emission properties and the work function determination of an array of conical carbon nanotubes" In preparation

2. Santoshrupa Dumpala, Abdelilah Safir, David Mudd, Robert W. Cohn, Mahendra K. Sunkara, and Gamini U. Sumanasekera; Controlled synthesis and Enhanced Field Emission Characteristics of Morphologically Varying Conical Carbon Nanotubular Arrays" Diamond and Related Materials 18, 10 (2009).

3. V. V. Dobrokhotov, D. N. Mcllroy, M. Grant Norton, R. Abdelrahaman, A. Safir and C. A. Berven, "Interaction of hybrid nanowire-nanoparticle structures with carbon monoxide" Nanotechnology 20 (2009).

4. Mehdi M. Yazdanpanah, Mahdi Hosseini, Santosh Pabba, Scott M. Berry, Vladimir V. Dobrokhotov, Abdelilah Safir, Robert S. Keynton and Robert W. Cohn "MicroWilhelmy and Related Liquid Property Measurements Using Constant Diameter Nanoneedle-Tipped AFM Probes" Langmuir, 2008, 24 (23).

5. Vladimir Dobrokhotov, Mehdi M. Yazdanpanah, Santosh Pabba, Abdelilah Safir and Robert W. Cohn, "Visual Force Sensing with Flexible Nanowire Buckling Springs" Nanotechnology 19 (2008).

6. M. M. Yazdanpanah S. A. Harfenist, A. Safir and R. W. Cohn, "Selective selfassembly at room temperature of individual freestanding Ag2Ga alloy nanoneedles" Journal of Applied Physics (2005).

7. Che-Yih Lim, Q. Huang, X. Xie, A. Safir, S. A. Harfenist, R. Cohn and E. J. Podlaha "Development of an Electrodeposited Nanomold from Compositionally Modulated Alloys" Journal of Applied Electrochemistry, 2004.

\section{REFEREED CONFERENCE PROCEEDINGS}

1. M.M. Yazdanpanah, M. Hosseini, A. Safir, S. Pabba, S.M. Berry, V.V. Dobrokhotov, R.S. Keynton, R.W. Cohn, "Probing Viscoelastic Properties of Single Cells Through 
Their Entire Thickness", SEM Annual Conference, Session 23 Nanomechanics in Nature, Massachusetts USA, June 3 - 6, 2007.

2. Vladimir Dobrokhotov, M. M. Yazdanpanah, Santosh Pabba, Abdelilah Safir \& Robert W Cohn "Elastic Buckling of Nanoscale Beams: Towards the Design of Nanomechanical Force-Sensor" SEM Annual Conference, Session 91 Nanocomposite Characterization, Massachusetts USA, June 3 - 6, 2007.

3. M.M. Yazdanpanah, M. Hosseini, S. Pabba, S.M. Berry, V.V. Dobrokhotov, A. Safir, R.S. Keynton, R.W. Cohn "Rheological Measurements by AFM of the Formation of Polymer Nanofibers" SEM Annual Conference, Session 91 Nanocomposite Characterization, Massachusetts USA, June 3 - 6, 2007.

4. A.Safir, M. M. Yazdanpanah, S. Pabba, S. D. Cambron, F. P. Zamborini, R. S. Keynton, and R. W. Cohn, "Fabrication of an insulated probe on a self-assembled metallic nanowire for electrochemical sensing inside cells," $20066^{\text {th }}$ IEEE Conference on Nanotechnology (19 July 2006, Cincinnati, OH).

\section{SELECTED CONFERENCE PRESENTATIONS AND TALKS}

1. Abdelilah Safir "Nanostructures as Electron Sources for Nanolithography and Nanomachining" Invited talk, University of Western Kentucky, Nov. 102008 Bowling Green, KY.

2. M.M. Yazdanpanah, V.V. Dobrokhotov, A. Safir, S. Pabba, D. Rojas and R.W. Cohn, "Room Temperature Growth of Single Intermetallic Nanostructures on Nanoprobes" NSTI Nanotech 2008.

3. Mehdi M. Yazdanpanah, M. Hosseini,S. Pabba, S. M. Berry, V. V. Dobrokhotov, A. Safir, D. Rojas, B. H. Fasciotto, R. S. Keynton, and R. W. Cohn "Selective selfassembly of metal nanoprobes for high aspect ratio SPM" Gordon Research Conference, Nanostructure fabrication" 13-18 July 2008 Tilton school NH

4. A. Safir, D. Mudd, M. M. Yazdanpanah, V. Dobrokhotov, Gamini Sumanasekera and R. W. Cohn "Field Emission Enhancement and the Field-Screening Effect Reduction using Carbon Nanopipettes as Cold Cathodes" APS Meeting March 10-14, 2008 New Orleans

5. Mehdi Yazdanpanah, Mahdi Hosseini, Santosh Pabba, Scott Berry, Vladimir Dobrokhotov, Abdelilah Safir, Robert Keynton, Robert Cohn "Directed Selfassembly of Nanostructures to Develop AFM-Based Biomaterial-to-Electronic Interface" APS Meeting March 10-14, 2008 New Orleans

6. Vladimir Dobrokhotov, Mehdi Yazdanpanah, Santosh Pabba, Abdelilah Safir, Robert Cohn "Mechanical Sensing with Flexible Metallic Nanowires" APS Meeting March 10-14, 2008 New Orleans

7. M. M. Yazdanapanah, M. Hosseini, S. Pabba, B. H. Fasciotto, S. M. Berry, V. V. Dobrokhotov, A. Safir, R. S. Keynton and R. W. Cohn "Rugged nanoneedle probes for live intracellular sensing, in-situ biomaterials synthesis and characterization of complex fluids" SAMPE Fall Technical Conference Cincinnati, OH. (Oct. 29 2007) 
8. M.M. Yazdanpanah, M. Hosseini, A. Safir, S. Pabba, S.M. Berry, V.V. Dobrokhotov, R.S. Keynton, R.W. Cohn, "Probing Viscoelastic Properties of Single Cells Through Their Entire Thickness", SEM Annual Conference, Session 23 Nanomechanics in Nature, Massachusetts USA, June 3 - 6, 2007

9. V.V. Dobrokhotov, M.M. Yazdanpanah, A. Safir, R.W. Cohn, "Comparing Generalized Elastica Model to Experimental Buckling Characteristics of Nanowires" SEM Annual Conference, Session 71 SYMPOSIUM MEMS \& NANOTECHNOLOGY: Nanoengineering, Massachusetts USA, June 3 - 6, 2007

10. A. Safir, M. M. Yazdanpanah, S. Pabba, V. Dobrokhotov, S. D. Cambron, F. P. Zamborini, R. S. Keynton and R. W. Cohn, "An insulated nanowire probe for electrochemical sensing in cells," ISOTT-2006, 34 ${ }^{\text {th }}$ Annual Conference of the International Society on Oxygen Transport to Tissue, Louisville, KY. (15 August 2006)

11. M. M. Yazdanpanah, A. Safir, S. Pabba, S.A. Harfenist, F. P. Zamborini and R. W. Cohn, "Nanostructure self-assembly by gallium-metal reactions at room temperature," Gordon Research Conference on Nanostructure Fabrication, Tilton, NH. (16-21 July 2006)

12. M. M. Yazdanpanah, S. A. Harfenist, A. Safir and R. W. Cohn, "Room Temperature, Selectively Self-Assembled, Freestanding Alloy Nanoneedles of $\mathrm{Ag}_{2} \mathrm{Ga}$ " Physical Electronics Conferences, Madison, WI, June 20, 2005.

13. R. W. Cohn, S. A. Harfenist, M. M. Yazdanpanah, A. Safir, T. Olaleye, P. Gopinath, S. Chakraborty, S. M. Berry, S. D. Cambron, E. A. Nelson, R. S. Keynton, B. W. Alphenaar, "Directed and Self-Assembled Nanwire Formation at Room Temperature For Rapid Nanoprototyping" Gordon Conference on Nanostructure Fabrication, Tilton NH, (18-23 July 2004) 